NATTONAL MNTTTUTE OF STANDABOS \&

TECHNOLOGY

Research Information Center

Gaithersburg, MD 20899 
NISTIR 85-3273-5

(Rev. 10/90)

\section{ENERGY PRICES AND DISCOUNT FACTORS FOR LIFE-CYCLE COST ANALYSIS 1991}

Annual Supplement to

NIST Handbook 135 and

NBS Special Publication 709

Data for the Federal Methodology for Life-Cycle Cost Analysis, Title 10, CFR, Part 436, Subpart A; and for the Energy Conservation Mandatory Performance Standards for New

Federal Residential Buildings, Title 10, CFR, Part 435

Barbara C. Lippiatt

Rosalie T. Ruegg

U.S. DEPARTMENT OF COMMERCE

National institute of Standards

and Technology

Applied Economics Group

Center for Computing and

Applied Mathematics

Gaithersburg, MD 20899

October 1990

Prepared for:

U.S. Department of Energy

Office of the Assistant Secretary for

Conservation and Renewable Energy

Federal Energy Management Program

Washington, DC 20585

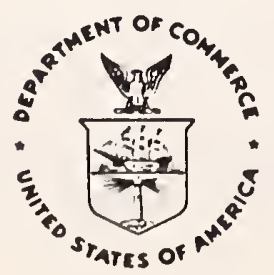





\section{PREFACE}

This is the 1991 edition of energy prices and discount factors for life-cycle cost analysis as established by the U.S. Department of Energy (DOE) in Subpart A of Part 436 of Title 10 of the Code of Federal Regulations (10 CFR Part 436, Subpart A), and amplified in the Life-Cycle Costing Manual for the Federal Energy Management Program (NIST Handbook 135). The data are provided as an aid to implementing life-cycle cost evaluations of potential energy conservation and renewable energy investments in existing and new federally owned and leased buildings.

The life-cycle costing methods and procedures as set forth in $10 \mathrm{CFR}$, Part 436, Subpart A, are to be followed by all Federal agencies, unless specifically exempted, in evaluating the cost effectiveness of potential energy conservation and renewable energy investments in federally owned and leased buildings.

As called for by legislation, the National Institute of Standards and Technology has provided technical assistance to the U.S. Department of Energy in the development and implementation of life-cycle costing methods and procedures. This is the second of a three-volume set which together provide the methods, data, and computational tools for life-cycle cost analysis of Federal energy projects.

Included in the three-volume set for Federal life-cycle cost analysis are the following:

\section{(1) Life-Cycle Costing Manual for the Federal Energy Management Program, National Institute of Standards and Technology, Handbook 135 (revised 1991).}

The manual is a guide to understanding life-cycle costing and related methods of economic analysis as they are applied to Federal decisions. It describes the required procedures and assumptions, defines and explains how to apply and interpret economic performance measures, gives examples of Federal decision problems and their solutions, explains how to use the energy price indices and discount factors which are updated annually in the supplement, and provides worksheets and other computational aids and instructions for calculating the required measures.

(2) Energy Prices and Discount Factors for Life-Cycle Cost Analysis,

National Institute of Standards and Technology, NISTIR 85-3273

(updated annually).

This report, which is updated annually, gives the energy price and discount factor multipliers needed to estimate the present value of energy and other future costs. The data are based on energy price projections developed by the Energy Information Administration of the U.S. Department of Energy. Request the latest edition when ordering. 
(3) NIST "Building Life Cycle Cost" (BLCC) Computer Program (version 3.0) National Institute of Standards and Technology (in press, 1990).

The NIST BLCC program, version 3.0, supercedes and incorporates both the Federal Buildings Life-Cycle Cost (FBLCC) and National Bureau of Standards Life-Cycle Cost (NBSLCC) programs. NIST BLCC is designed to run on IBM PC and compatible microcomputers with approximately $512 \mathrm{~K}$ of random access memory (RAM). It can be used to calculate the LCC of capital investments in buildings and building systems which are intended to reduce future operating, maintenance, and energy costs. BLCC computes the LCC for each alternative, compares alternatives in order to determine which has the lowest LCC, performs cash flow analyses, and then computes the net savings, savings-to-investment ratio (SIR), and adjusted internal rate of return (AIRR) over the designated study period. BLCC can be used to perform economic analysis of Federal and of private sector projects. BLCC version 3.0 uses the 1991 energy price data in NISTIR 85-3273-5. BLCC in its application to Federal energy conservation and renewable energy projects is consistent with NIST Handbook 135 (see \#1 above). In its application to non-energy projects, BLCC is consistent with OMB Circular A-94. In its application to private-sector and non-Federal public-sector projects, BLCC is consistent with ASTM standards for building economics. BLCC is integrated with the DOE ASEAM computer program which performs energy conservation analysis.

Included on the BLCC disk is a stand-alone program called DISCOUNT version 2.0 which can calculate present value, future value, and annual value factors for any discount rate and study period. DISCOUNT can access the DOE energy price projections included on the BLCC disk to compute the UPW* factors needed for Federal LCC analyses of energy projects, consistent with the factors included in this report.

The three-volume set can also be used to perform economic evaluations of Federal building projects which are not primarily for conserving energy or providing renewable energy but which have an energy cost component. Handbook 135 explains both applications.

The U.S. Department of Energy was directed by legislation and executive order to make available to the private sector the methods, procedures, and related aids developed for Federal use. In response to this directive, the National Institute of Standards and Technology, under sponsorship by the U.S. Department of Energy, published a life-cycle costing book for use by the private sector entitled Comprehensive Guide for Least-Cost Energy Decisions, NBS SP 709 (January 1987). The private sector guide is supported by the data provided here, as well as by the BLCC computer program. The BLCC program (version 3.0) supercedes the NBSLCC program which is documented in SP 709. BLCC provides LCC computational support for private sector projects as well as for Federal projects. 
To order any of the printed publications contact:

Advanced Sciences, Inc.

2000 North 15 th Street

Suite 407

Arlington, VA 22201

Telephone (703) 243-4900

Please request the publications by name and number.

To order BLCC for analyses of Federal buildings, contact the above address. Other users may order BLCC from one of the following:

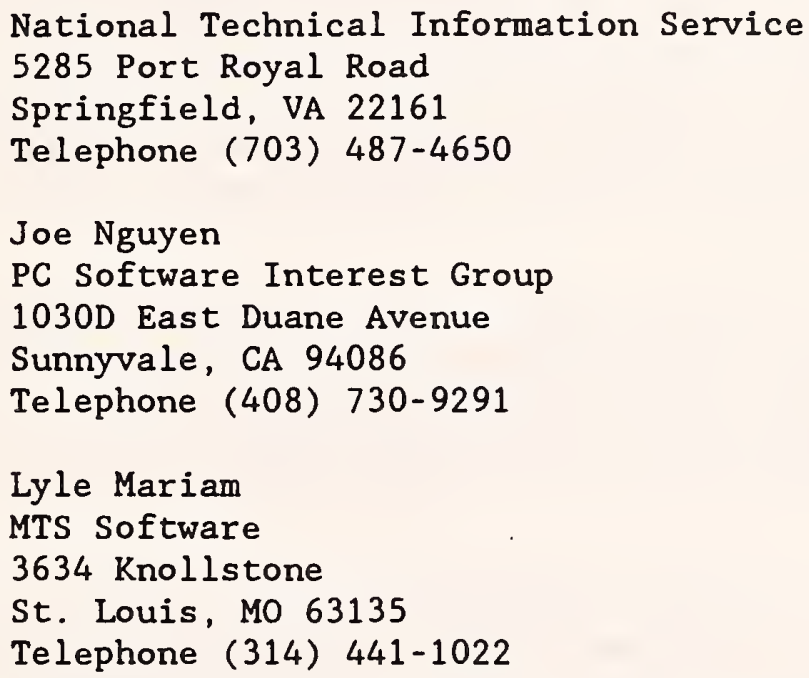

Workshops on the life-cycle costing method and energy analysis are conducted at locations around the country each year. The workshops include training and software for both BLCC and an energy analysis computer program called "A Simplified Energy Analysis Method" (ASEAM). A schedule of workshops can be obtained from the Applied Economics Group, National Institute of Standards and Technology, Bldg. 101, Rm. A415, Gaithersburg, MD 20899, Telephone (301) 975-6132.

An introduction to the workshop is provided in a video training film, "LeastCost Energy Decisions: An Introduction to Life-Cycle Cost Analysis." The video film and companion workbook can be ordered from Video Transfer, Inc., 5709-B Arundel Avenue, Rockville, MD 20852, Tel (301) 881-0270. Additional video training films in the series are in preparation.

Further information on the Federal Energy Management Program can be obtained from the Federal Energy Management Program Staff, Office of the Assistant Secretary for Conservation and Renewable Energy, U.S. Department of Energy. Please direct communication to: FEMP, CE 10.1, U.S. Department of Energy, 1000 Independence Avenue, SW, Washington, D.C. 20585. 
Please note that Federal Methodology for Life-Cycle Cost Analysis, Title 10, CFR, Part 436, Subpart A has been revised to incorporate changes required by the Federal Energy Management Improvement Act of 1988 (P.L. 100-615), and to reflect 10-years experience with the Federal LCC Rule.

The principal change is a discount rate set annually by DOE. The rate for 1991 is equivalent to a market rate of 8.48 and is based on long-term Treasury bond rates averaged over the previous 12 months. The market rate is converted to a "real" discount rate of 4.7\%, exclusive of the Administration's assumed rate of general price inflation, to correspond with the constant-dollar analysis approach that is used. The results are identical to those that would be obtained by using the 8.48 market rate as the discount rate and inflating all cash flows at the Administration's assumed rate of inflation. (For further discussion of changes in the Federal life-cycle costing rule see Notice of Final Rulemaking, Federal Register, October 31, 1990. For a more detailed description of how the Federal discount rate is determined, see NIST Handbook 135 (revised 1991).)

The SPW, UPW, and UPW factors in Part I of this report are given both for the 4.78 discount rate and for a $10 \%$ discount rate. The former are for evaluating Federal energy conservation and renewable energy projects. The latter are for evaluating Federal projects subject to OMB Circular A-94, i.e., most Federal capital investment projects other than energy projects and water-resources projects.

An additional change in the Federal methodology that affects the data in this report is the allowance in the analysis of a planning/design/construction period prior to building occupancy. The text that accompanies the "B" series of tables explains how to use the UPW factors to account for a planning/design/construction period. 


\section{ABSTRACT}

This is the 1991 annual edition of energy prices and discount factors for performing life-cycle cost analyses of energy conservation and renewable energy projects. It supports the Federal life-cycle costing methodology by updating the energy price projections and discount factors that are described, explained, and illustrated in NIST Handbook 135 (HB 135). It supports private-sector life-cycle cost analysis by updating the energy price indices that are described, explained, and illustrated in NBS Special Publication 709 (SP 709). It also supports the Energy Conservation Mandatory Performance Standards for New Federal Residential Buildings (10 CFR 435) by providing a table of factors for updating appliance label values. 
The authors wish to thank Mr. Richard W. Brancato and Mr. K. Dean Devine of the Federal Energy Management Program of the U.S. Department of Energy (DOE) for their continued support and direction of this work. Appreciation is also extended to Dr. Gerald E. Peabody and Mr. Michael D. Lehr of the Supply Analysis and Integration Branch of the DOE Energy Information Administration for providing the energy price projections and escalation rates upon which this report is based. 
CONTENTS

Page

PREFACE . . . . . . . . . . . . . . . . . . . . . . . iii

NOTICE. . . . . . . . . . . . . . . . . . . . . . . . . vi

ABSTRACT. . . . . . . . . . . . . . . . . . . . . . vii

ACKNOWLEDGMENTS . . . . . . . . . . . . . . . . . . . . . . . . viii

CONTENTS. . . . . . . . . . . . . . . . . . . . . . . . ix

LIST OF TABLES . . . . . . . . . . . . . . . . . . . . $\mathrm{x}$

ABBREVIATIONS . . . . . . . . . . . . . . . . . . . . . . . . xiv

PART I: TABLES FOR FEDERAL LIFE-CYCLE COST ANALYSIS . . . . . . . . . . 1

Single Present Worth and Uniform Present Worth discount factors . . . . 1

Modified Uniform Present Worth discount factors for Federal use . . . . 5

Projected average fuel price indices and escalation rates for Federal

use............................ 18

Factors for updating appliance label values . . . . . . . . . . . 35

PART II: TABLES FOR PRIVATE SECTOR LIFE-CYCLE COST ANALYSIS . . . . . . 36

Projected average fuel price indices for private sector use . . . . . . 36 
A-1. SPW factors for finding the present value of future nonfuel, nonannually recurring amounts .. . . . . . . . . . . . 3

A-2. UPW factors for finding the present value of future nonfuel, annually recurring amounts. . . . . . . . . . . . . . . 4

B-1a. UPW discount factors adjusted for average fuel price escalation, by end-use sector and major fuel. Discount rate $=4.7$ percent. Census Region 1 (Maine, New Hampshire, Vermont, Massachusetts, Connecticut, Rhode Island, New York, New Jersey, Pennsylvania) . . . . . . . . . . . . . .

B-2a. UPW" discount factors adjusted for average fuel price escalation, by end-use sector and major fuel. Discount rate $=4.7$ percent. Census Region 2 (Ohio, Indiana, Illinois, Michigan, Wisconsin, Minnesota, Iowa, Missouri, North Dakota, South Dakota, Nebraska, Kansas) . . . . . . . . . . . . .

B-3a. UPW discount factors adjusted for average fuel price escalation, by end-use sector and major fuel. Discount rate $=4.7$ percent. Census Region 3 (Delaware, Maryland, District of Columbia, Virginia, West Virginia, North Carolina, South Carolina, Georgia, Florida, Kentucky, Tennessee, Alabama, Mississippi, Arkansas, Louisiana, Oklahoma, Texas).

B-4a. UPW" discount factors adjusted for average fuel price escalation, by end-use sector and major fuel. Discount rate $=4.7$ percent. Census Region 4 (Montana, Idaho, Wyoming, Colorado, New Mexico, Arizona, Utah, Nevada, Washington, Oregon, California, Alaska, Hawaii) . . . . . . . . . . .

B-5a. $\quad \mathrm{UPW}^{*}$ discount factors adjusted for average fuel price escalation, by end-use sector and major fuel. Discount rate $=4.7$ percent. United States average. . . . . . . .

B-1b. UPW* discount factors adjusted for average fuel price escalation, by end-use sector and major fuel. Discount rate $=10$ percent. Census Region 1 (Maine, New Hampshire, Vermont, Massachusetts, Connecticut, Rhode Island, New York, New Jersey, Pennsylvania) . . . . . . . . . . . . . 13

B-2b. UPW discount factors adjusted for average fuel price escalation, by end-use sector and major fuel. Discount rate $=10$ percent. Census Region 2 (Ohio, Indiana, Illinois, Michigan, Wisconsin, Minnesota, Iowa, Missouri, North Dakota, South Dakota, Nebraska, Kansas) . . . . . . . 14 
B-3b. UPW ${ }^{\star}$ discount factors adjusted for average fuel price escalation, by end-use sector and major fuel. Discount rate $=10$ percent. Census Region 3 (Delaware, Maryland, District of Columbia, Virginia, West Virginia, North Carolina, South Carolina, Georgia, Florida, Kentucky, Tennessee, Alabama, Mississippi, Arkansas, Louisiana, Oklahoma, Texas).

B-4b. UPW* discount factors adjusted for average fuel price escalation, by end-use sector and major fuel. Discount rate $=10$ percent. Census Region 4 (Montana, Idaho, Wyoming, Colorado, New Mexico, Arizona, Utah, Nevada, Washington, Oregon, California, Alaska, Hawaii) . . . . . 16

B-5b. UPW ${ }^{*}$ discount factors adjusted for average fuel price escalation, by end-use sector and major fuel. Discount rate $=10$ percent. United States average . . . . . . . . .

Ca-1. Projected average fuel price indices tied to the value of the dollar in 1991, by end-use sector and major fuel.

Census Region 1 (Maine, New Hampshire, Vermont,

Massachusetts, Connecticut, Rhode Island, New York, New Jersey, Pennsylvania)

Ca-2. Projected average fuel price indices tied to the value of the dollar in 1991, by end-use sector and major fuel. Census Region 2 (Ohio, Indiana, Illinois, Michigan, Wisconsin, Minnesota, Iowa, Missouri, North Dakota, South Dakota, Nebraska, Kansas). . . . . . . . . . . .

Ca-3. Projected average fuel price indices tied to the value of the dollar in 1991, by end-use sector and major fuel. Census Region 3 (Delaware, Maryland, District of Columbia, Virginia, West Virginia, North Carolina, South Carolina, Georgia, Florida, Kentucky, Tennessee, Alabama, Mississippi, Arkansas, Louisiana, Oklahoma, Texas). . . . . . . . . . 24

Ca-4. Projected average fuel price indices tied to the value of the dollar in 1991, by end-use sector and major fuel.

Census Region 4 (Montana, Idaho, Wyoming, Colorado, New Mexico, Arizona, Utah, Nevada, Washington, Oregon, California, Alaska, Hawaii) 
Ca-5. Projected average fuel price indices tied to the value of the dollar in 1991, by end-use sector and major fuel.

United States average . . . . . . . . . . . . . . .

Cb-1. Projected average fuel price escalation rates exclusive of general price inflation, by end-use sector and major fuel (percentage change compounded annually). Census Region 1 (Maine, New Hampshire, Vermont, Massachusetts, Connecticut, Rhode Island, New York, New Jersey, Pennsylvania) . . . . .

$\mathrm{Cb}-2$. Projected average fuel price escalation rates exclusive of general price inflation, by end-use sector and major fuel (percentage change compounded annually). Census Region 2 (Ohio, Indiana, Illinois, Michigan, Wisconsin, Minnesota, Iowa, Missouri, North Dakota, South Dakota, Nebraska,

Kansas) . . . . . . . . . . . . . . . . . . . .

Cb-3. Projected average fuel price escalation rates exclusive of general price inflation, by end-use sector and major fuel (percentage change compounded annually). Census Region 3 (Delaware, Maryland, District of Columbia, Virginia, West Virginia, North Carolina, South Carolina, Georgia, Florida, Kentucky, Tennessee, Alabama, Mississippi, Arkansas, Louisiana, Oklahoma, Texas).

$\mathrm{Cb}-4$. Projected average fuel price escalation rates exclusive of general price inflation, by end-use sector and major fuel (percentage change compounded annually). Census Region 4 (Montana, Idaho, Wyoming, Colorado, New Mexico, Arizona, Utah, Nevada, Washington, Oregon, California, Alaska, Hawaii). . . . . . . . . . . . . . . . . .

Cb-5. Projected average fuel price escalation rates exclusive of general price inflation, by end-use sector and major fuel (percentage change compounded annually). United States average . . . . . . . . . . . . . . . . 34

D. Factors for updating appliance label values . . . . . . . 35

S-1. Projected average fuel price indices with assumed general price inflation rates of $0,2.5,5$, and 7.5 percent, by enduse sector and major fuel. Census Region 1 (Maine, New Hampshire, Vermont, Massachusetts, Connecticut, Rhode Island, New York, New Jersey, Pennsylvania) . . . . . . . 38 
S-2. Projected average fuel price indices with assumed general price inflation rates of $0,2.5,5$, and 7.5 percent, by enduse sector and major fuel. Census Region 2 (Ohio, Indiana, Illinois, Michigan, Wisconsin, Minnesota, Iowa, Missouri, North Dakota, South Dakota, Nebraska, Kansas). . . . . . . 40

S-3. Projected average fuel price indices with assumed general price inflation rates of $0,2.5,5$, and 7.5 percent, by enduse sector and major fuel. Census Region 3 (Delaware, Maryland, District of Columbia, Virginia, West Virginia, North Carolina, South Carolina, Georgia, Florida, Kentucky, Tennessee, Alabama, Mississippi, Arkansas, Louisiana,

Oklahoma, Texas). . . . . . . . . . . . . . . 42

S-4. Projected average fuel price indices with assumed general price inflation rates of $0,2.5,5$, and 7.5 percent, by enduse sector and major fuel. Census Region 4 (Montana, Idaho, Wyoming, Colorado, New Mexico, Arizona, Utah, Nevada, Washington, Oregon, California, Alaska, Hawaii) . . . . . 44

S-5. Projected average fuel price indices with assumed general price inflation rates of $0,2.5,5$, and 7.5 percent, by enduse sector and major fuel. United States average . . . . . 46 


\section{ABBREVIATIONS}

N

- Number of Discount Periods (in Years)

ELEC - Electricity

DIST - Distillate Oil

LPG - Liquefied Petroleum Gas

NTGAS - Natural Gas

RESID - Residual Oil

COAL - Steam Coal

GASLNE - Gasoline 
Single Present Worth and Uniform Present Worth discount factors

Table A-1 presents the single present worth (SPW) factors for finding the present value of future nonfuel, nonannually recurring amounts, such as repair and replacement costs and salvage values. The formula for finding the present value $(P)$ of a future amount $(F)$ is the following:

$$
P=F \times \frac{1}{(1+d)^{N}}=F \times S P W_{N} \text { Factor, }
$$

where $\mathrm{d}=$ discount rate; and

$\mathrm{N}=$ number of periods, such as years, until $\mathrm{F}$ occurs.

Table A-2 presents uniform present worth (UPW) factors for finding the present value of future nonfuel amounts recurring annually, such as routine maintenance costs. The formula for finding the present value $(P)$ of an annually recurring uniform amount (A) is the following:

$$
P=A \times \frac{(1+d)^{N}-1}{d(1+d)^{N}}=A \times U^{N} W_{N} \text { Factor, }
$$

where $\mathrm{N}=$ number of periods, such as years, over which $\mathrm{A}$ recurs.

In tables $A-1$ and $A-2$ the factors are given for both 4.7 percent and 10 percent discount rates. The factors based on 4.7 percent are for finding the present value of future amounts associated with Federal energy conservation and renewable energy projects. The factors based on 10 percent are for finding the present value of future amounts associated with most other Federal projects (except those specifically exempted from the 10 percent rate). Both the 4.7 and 10 percent rates are defined for Federal use to be "real" rates exclusive of general price inflation. The factors are applied as multipliers to future amounts which are stated in "constant" dollars, that is, exclusive of general price inflation.

\section{Examples of How to Use the Factors:}

SPW(4.78): To compute the present value of a replacement cost expected to occur in the 8 th year for an energy efficient heating system, go to table A-1, find the 4.7 percent SPW factor for year $8(0.69)$, and multiply the factor by the replacement cost stated in 1991 dollars.

SPW(108): To compute the present value of a repair cost in the 5 th year for a floor covering (non-energy related), go to table A-1, find the 10 percent SPW factor for year $5(0.62)$, and multiply the factor by the repair cost stated in 1991 dollars. 
UPW( 4.78$)$ : To compute the present value of an annually recurring maintenance cost for a renewable energy system over 20 years, go to table A-2, find the 4.7 percent UPW factor for 20 years (12.79), and multiply the factor by the annual maintenance cost stated in 1991 dollars.

UPW(10\%): To compute the present value of annually recurring costs of office cleaning over 30 years (for a project not primarily related to energy consumption), go to table A-2, find the 10 percent UPW factor for 30 years (9.43), and multiply the factor by the annual cleaning cost stated in 1991 dollars.

For further explanation and illustration of how to use these factors, see NIST Handbook 135. 
Table A-1. SPW factors for finding the present value of future nonfuel, nonannually recurring amounts

\begin{tabular}{|c|c|c|}
\hline $\begin{array}{l}\text { Number of Years } \\
\text { Until Future Amount } \\
\text { Occurs (N) }\end{array}$ & $\begin{array}{l}\text { FEMP LCC Approach } \\
\text { SPW Factor } \\
(d=4.7 \%)\end{array}$ & $\begin{array}{l}\text { OMB A-94 LCC Approach } \\
\text { SPW Factor } \\
(d=10 \%)\end{array}$ \\
\hline 1 & 0.96 & 0.91 \\
\hline 2 & 0.91 & 0.83 \\
\hline 3 & 0.87 & 0.75 \\
\hline 4 & 0.83 & 0.68 \\
\hline 5 & 0.79 & 0.62 \\
\hline 6 & 0.76 & 0.56 \\
\hline 7 & 0.73 & 0.51 \\
\hline 8 & 0.69 & 0.47 \\
\hline 9 & 0.66 & 0.42 \\
\hline 10 & 0.63 & 0.39 \\
\hline 11 & 0.60 & 0.35 \\
\hline 12 & 0.58 & 0.32 \\
\hline 13 & 0.55 & 0.29 \\
\hline 14 & 0.53 & 0.26 \\
\hline 15 & 0.50 & 0.24 \\
\hline 16 & 0.48 & 0.22 \\
\hline 17 & 0.46 & 0.20 \\
\hline 18 & 0.44 & 0.18 \\
\hline 19 & 0.42 & 0.16 \\
\hline 20 & 0.40 & 0.15 \\
\hline 21 & 0.38 & 0.14 \\
\hline 22 & 0.36 & 0.12 \\
\hline 23 & 0.35 & 0.11 \\
\hline 24 & 0.33 & 0.10 \\
\hline 25 & 0.32 & 0.09 \\
\hline $26^{\star}$ & 0.30 & 0.08 \\
\hline $27^{*}$ & 0.29 & 0.08 \\
\hline $28 *$ & 0.28 & 0.07 \\
\hline $29^{\prime \prime}$ & 0.26 & 0.06 \\
\hline $30^{*}$ & 0.25 & 0.06 \\
\hline
\end{tabular}


Table A-2. UPW factors for finding the present value of future nonfuel, annually recurring amounts

\begin{tabular}{|c|c|c|}
\hline $\begin{array}{l}\text { Number of Years } \\
\text { Over Which Amount } \\
\text { Recurs (N) }\end{array}$ & $\begin{array}{l}\text { FEMP LCC Approach } \\
\text { UPW Factor } \\
\text { (d=4.7\%) }\end{array}$ & $\begin{array}{l}\text { OMB A-94 LCC Approach } \\
\text { UPW Factor } \\
(d=10 \%)\end{array}$ \\
\hline 1 & 0.96 & 0.91 \\
\hline 2 & 1.87 & 1.74 \\
\hline 3 & 2.74 & 2.49 \\
\hline 4 & 3.57 & 3.17 \\
\hline 5 & 4.37 & 3.79 \\
\hline 6 & 5.12 & 4.36 \\
\hline 7 & 5.85 & 4.87 \\
\hline 8 & 6.54 & 5.33 \\
\hline 9 & 7.20 & 5.76 \\
\hline 10 & 7.84 & 6.14 \\
\hline 11 & 8.44 & 6.50 \\
\hline 12 & 9.02 & 6.81 \\
\hline 13 & 9.57 & 7.10 \\
\hline 14 & 10.09 & 7.37 \\
\hline 15 & 10.59 & 7.61 \\
\hline 16 & 11.07 & 7.82 \\
\hline 17 & 11.53 & 8.02 \\
\hline 18 & 11.97 & 8.20 \\
\hline 19 & 12.39 & 8.36 \\
\hline 20 & 12.79 & 8.51 \\
\hline 21 & 13.17 & 8.65 \\
\hline 22 & 13.53 & 8.77 \\
\hline 23 & 13.88 & 8.88 \\
\hline 24 & 14.21 & 8.98 \\
\hline 25 & 14.53 & 9.08 \\
\hline $26 *$ & 14.83 & 9.16 \\
\hline $27^{\star}$ & 15.12 & 9.24 \\
\hline $28^{*}$ & 15.40 & 9.31 \\
\hline $29^{*}$ & 15.66 & 9.37 \\
\hline $30^{*}$ & 15.91 & 9.43 \\
\hline
\end{tabular}


Modified Uniform Present Worth discount factors for Federal use (Based on Federally required discount rates of 4.7 and 10 percent and DOEprojected rates of change in energy prices, both of which exclude general price inflation)

This section presents "modified" uniform present worth (UPW") discount factors for the 4 Census regions and for the United States. The factors are modified in the sense that they incorporate projected energy price changes. There are two sets of tables: the "a" tables present UPW" factors based on a 4.7 percent "real" discount rate, and the "b" tables present UPW" factors based on a 10 percent real discount rate.

The factors presented in the "a" tables are for calculating the present value of energy costs or savings accruing over 1 to 25 years and are to be used in life-cycle cost analyses of Federal energy conservation and renewable energy projects. Factors are now reported in the "a" tables for 30 years to accommodate a planning/design/construction period of up to 5 years. (See "Example of How to Use UPW" Factors" below for instructions on use with planning/design/construction periods.)

The factors presented in the "b" tables are for calculating the present value of energy costs or savings accruing over 1 to 30 years and are to be used for life-cycle cost analysis of the energy component of Federal projects that are not primarily for conserving energy or providing renewable energy. Both sets of factors apply only to energy costs and savings that recur in uniform amounts. Refer to NIST Handbook 135 for evaluating energy costs and savings that vary in amount over time.

The UPW" factors incorporate rates of change in energy prices computed from indices projected by the Energy Information Administration (EIA) of the U.S. Department of Energy. The EIA data are stated as annual averages. Therefore, the factors are not tied to a particular calendar date in the year.

Documentation on the PC-AEO model used by EIA to project energy prices is available in PC-AEO Forecasting Model for the Annual Energy Outlook 1990 (DOE/EIA-M036(90)), March 1990. Assumptions underlying the PC-AEO model are presented by EIA in Assumptions for the Annual Energy Outlook 1990 (DOE/EIA $0527(90)$ ), February 1990. Projections at the national level are reported by EIA in the Annual Energy Outlook 1990 (DOE/EIA-0383(90)), January 1990.

Note that the EIA energy price projections predate the 1990 crisis with Iraq and do not reflect the ensuing sharp spurt in oil prices. The user is reminded, however, that Federal agencies are to use their actual energy prices as of the date the analysis is performed as the starting point for estimating present value energy costs. It is expected that this practice will account for the jump in prices that followed release of the price projections.

The formula for finding the present value (P) of future energy costs or savings is the following:

$$
P=A_{0} \times \sum_{n=1}^{N} \frac{I_{(19 \theta 1+n)}}{(1+d)^{n}}=A_{0} \times U P W^{*} \text { Factor, }
$$


where $A_{0}$ = base-year dollar cost of energy, i.e., the annual quantity of energy times its 1991 price;

$\mathrm{n}$ = counter used to designate each year, with $\mathrm{n}=1$ for the year 1992;

$\mathrm{N}$ = number of periods, e.g. years, over which energy costs or savings accrue;

$I_{(1991+n)}=$ projected average fuel price index given in tables Ca-1 through $\mathrm{Ca}-5$ for the year $1991+\mathrm{n}^{1}$; and

$\mathrm{d}=$ discount rate.

Examples of How to Use UPW* Factors:

$\mathrm{UPW}^{*}(4.78$, no planning/design/construction period): To compute the present value of heating with distillate oil over 15 years for an energy-conserving design of a Federal office building in New Mexico, go to table B-4a, find the UPW factor for commercial distillate for 15 years (14.19), and multiply the factor by the annual heating cost in 1991 dollars.

$\mathrm{UPW}^{*}$ (4.78, with planning/design/construction period): (1) Find the UPW* factor for the combined lengths of the planning/design/construction period and the occupancy period (not to exceed 30 years), and (2) subtract from (1) the $\mathrm{UPW}^{*}$ factor for the planning/design/construction period alone. The difference is the UPW ${ }^{\star}$ factor for the years over which energy costs or savings accrue. For example, suppose an energy-conserving Federal office building in New York is being designed. It is expected to have a planning/design/construction period of 5 years, after which it will be occupied for at least 25 years. To compute the present value of natural gas costs over 25 years of occupancy, go to table B-la and find the UPW factors for commercial natural gas for 5 years (4.51) and for 30 years (21.17). The difference (16.66) is the UPW factor for natural gas costs over 25 years, beginning 5 years hence. Multiply 16.66 by the annual natural gas cost in 1991 dollars to calculate the present value of natural gas costs over the study period.

UPW $^{\star}(108)$ : To compute the present value of electricity costs over 30 years associated with rehabilitating a Federal office building in Ohio (energy conservation is not a primary objective), go to table $B-2 b$, find the UPW factor for commercial electricity for 30 years (9.59), and multiply by the annual electricity cost in 1991 dollars.

For further explanation of the use of UPW ${ }^{\star}$ factors, see NIST Handbook 135.

Note: The data in the tables which follow are reported by 4 Census regions. Figure B-1 presents a map showing the states corresponding to the 4 Census regions. The Census regions do not include American Samoa, Canal Zone, Guam, Puerto Rico, Trust Territory of the Pacific Islands, or the Virgin Islands. Analysts of Federal projects in these areas should use data which are "reasonable under the circumstances," and may refer to the tables with U.S. average data for guidance.

${ }^{1}$ For greater precision, the UPW* factors reported in the $\mathrm{Ba}$ and $\mathrm{Bb}$ tables were computed using the unrounded form of the indices given in tables $\mathrm{Ca}-1$ through $\mathrm{Ca}-5$. 


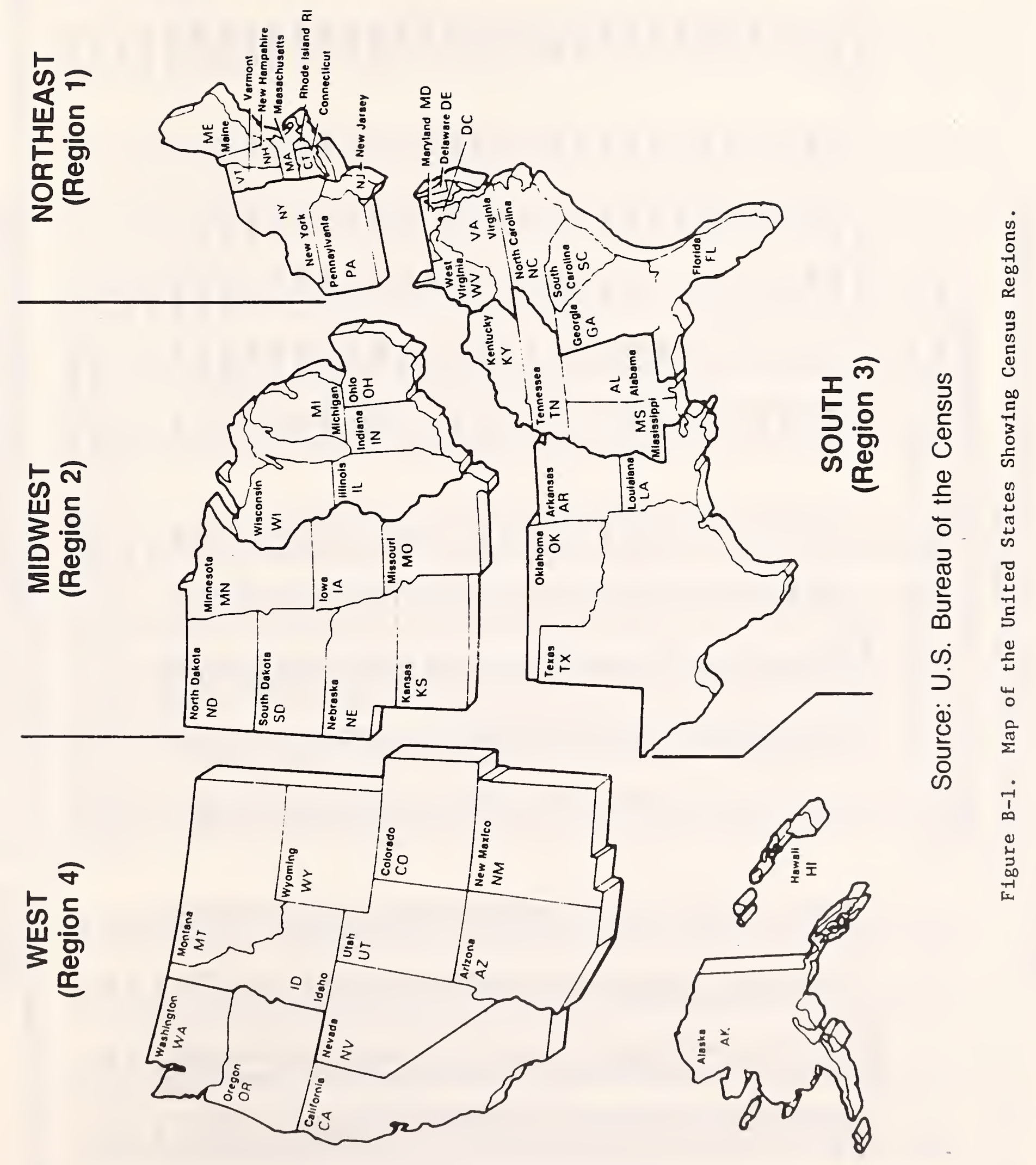


ż기에 

引

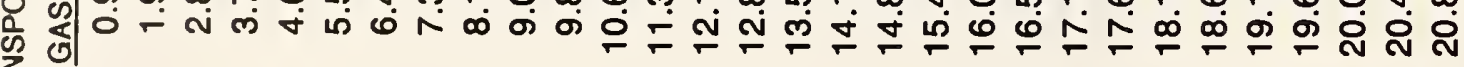
点

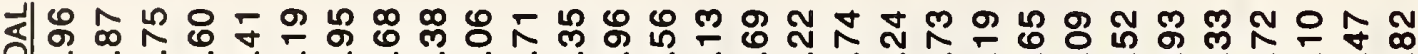
U|O-

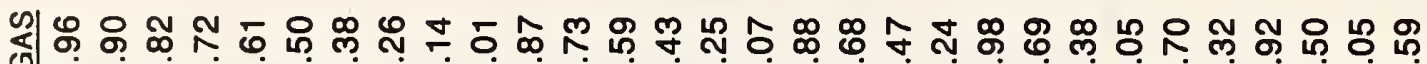
।

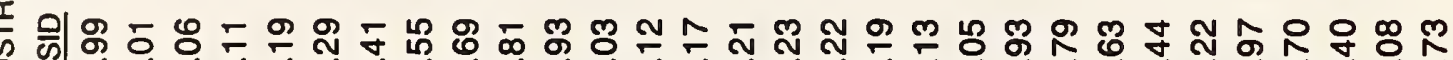
凯

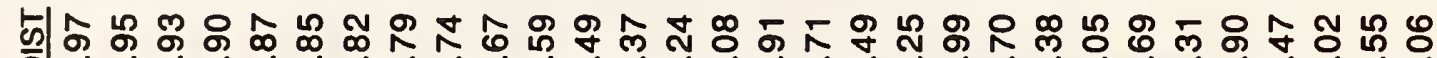
D)

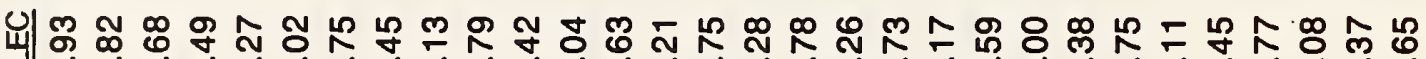
Uు

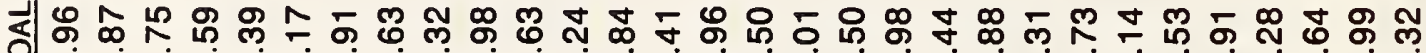
O|이 -

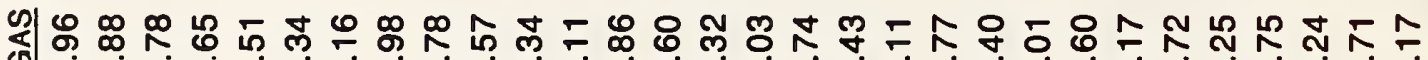
$\leq \mathrm{C}^{\circ}$

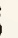

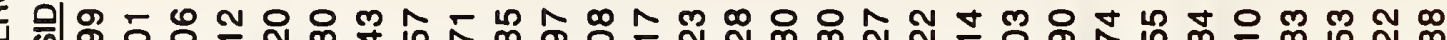
क $00 \%$, 近

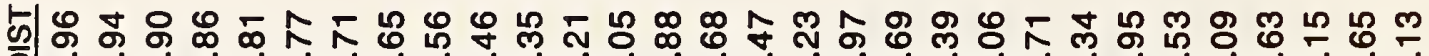
0)

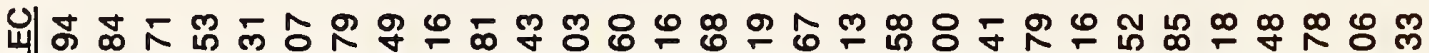
Wا

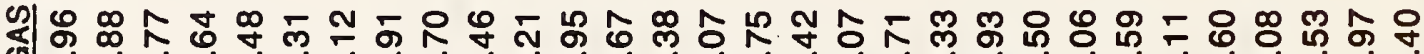

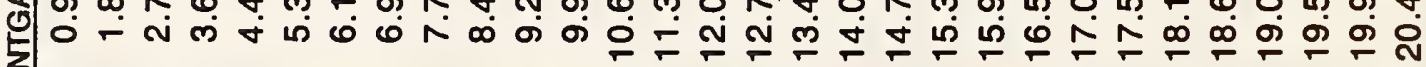

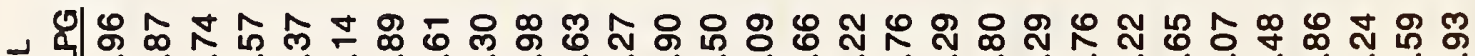
可

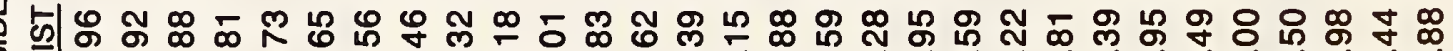
崖古宁

仙

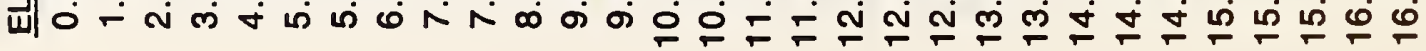

z| 


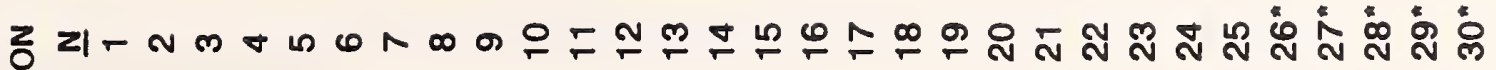
$\stackrel{E}{E}$

营 传 点

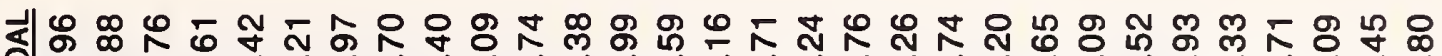

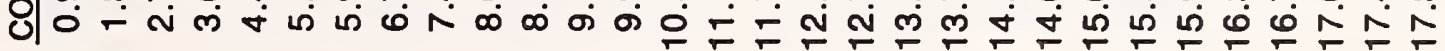

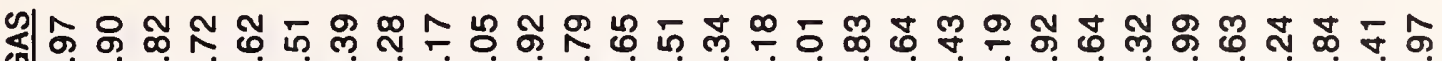
ل|

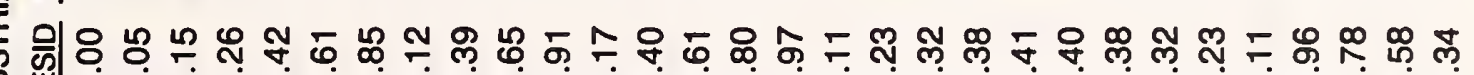

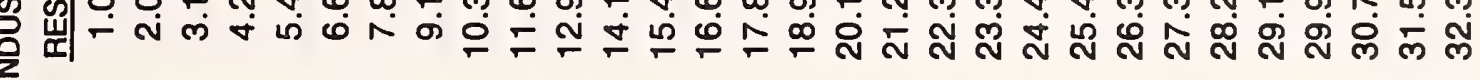

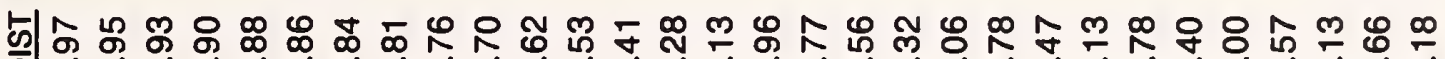
ప|

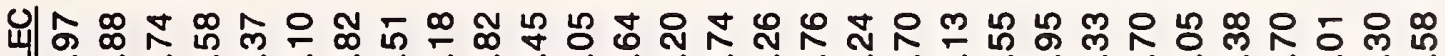
Шالَ

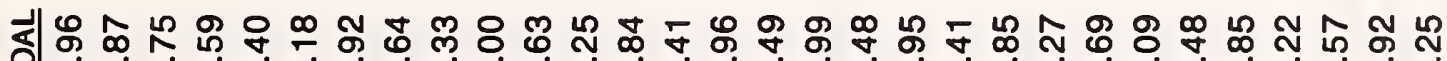
ठإن-

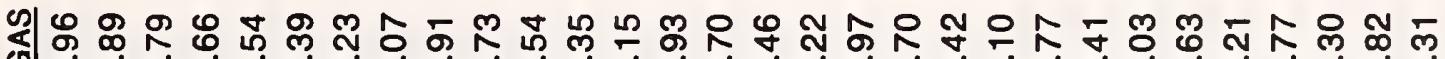

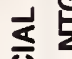

O-N m

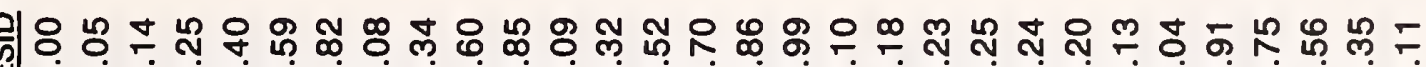

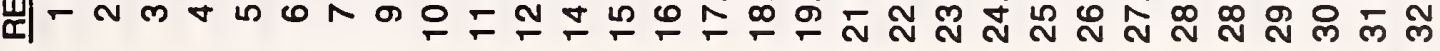
勾。 0 仙 嵌

ஊุด 年 丩|이유

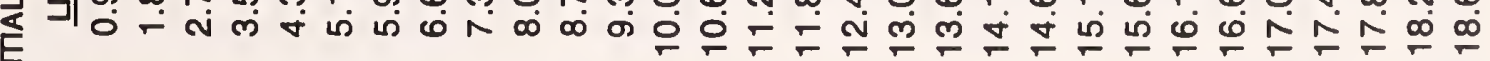
$\sum$

岂 岁苛

朔合市 Шا山

Z1-Nm+แ 
Z

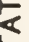

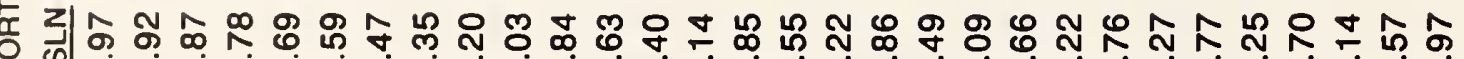
के O-

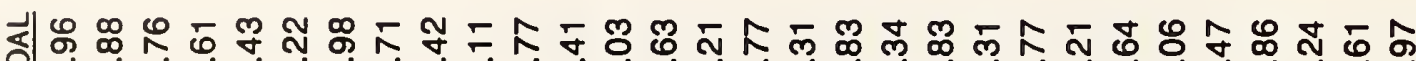
ర|ن-

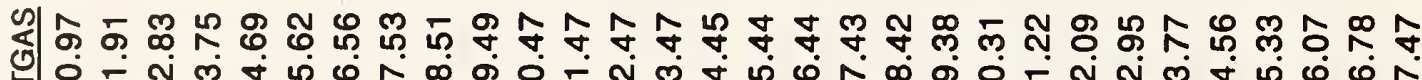
\& เి

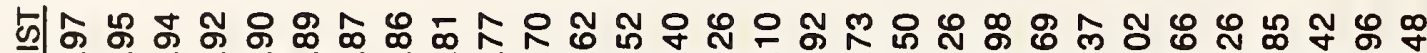
ప

仙尔

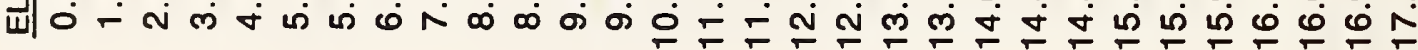

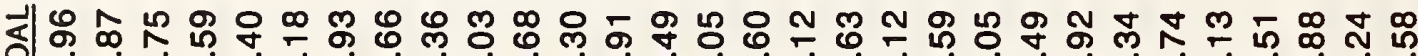

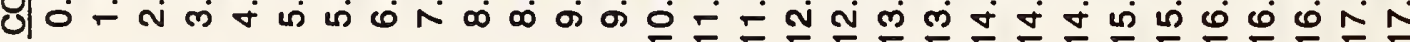

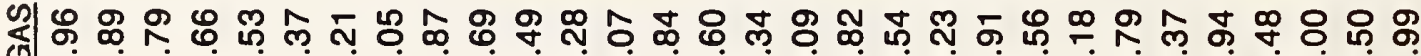
衣 穴

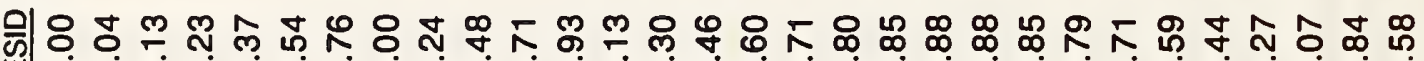
湈-

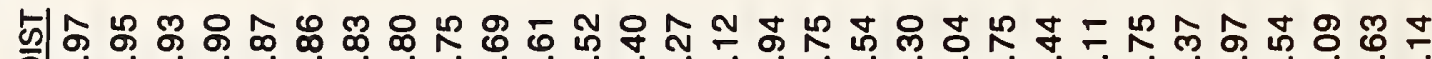
ప。

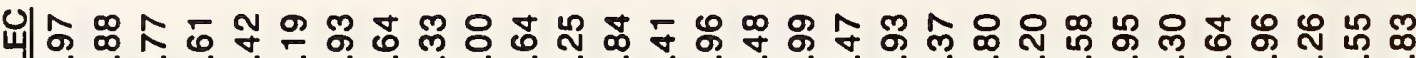
Uا山

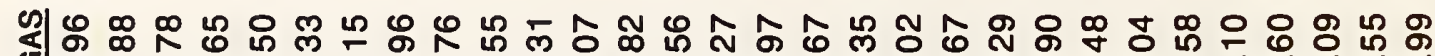
氙 -

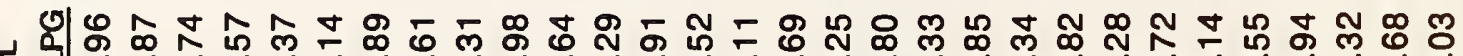

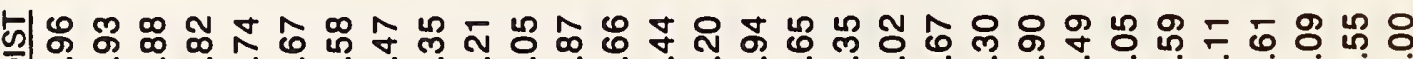

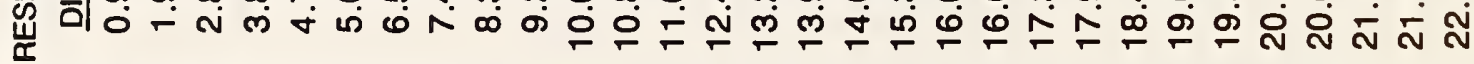

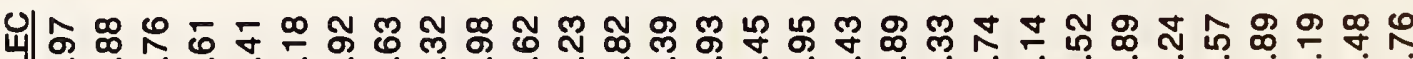
岒

z| 
z 产

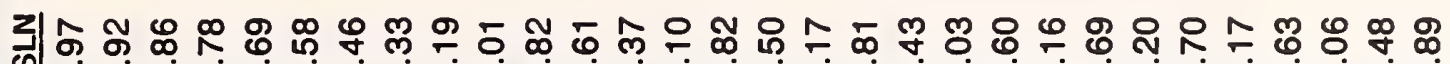
के

⿷匚⿳丨コ丨

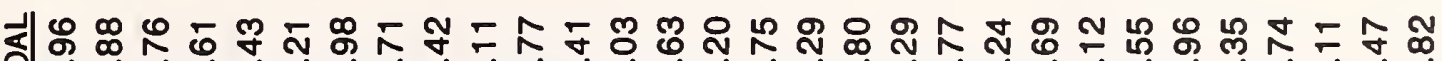

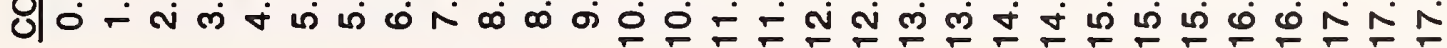

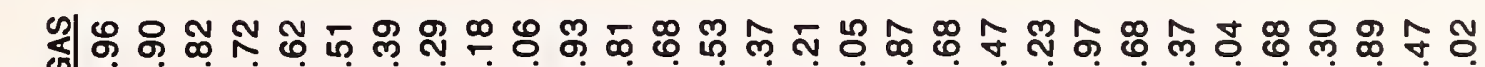

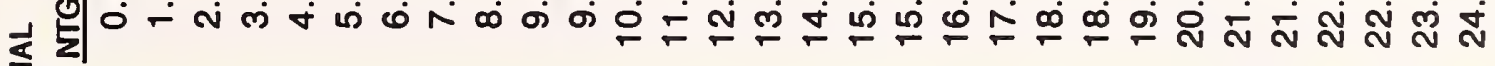

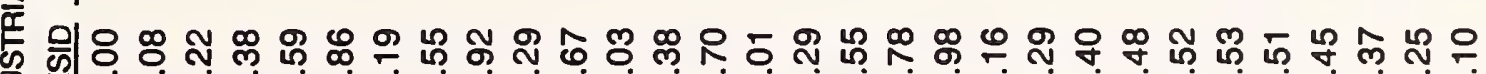
㟢一 ⿹勹厶 ||

仙

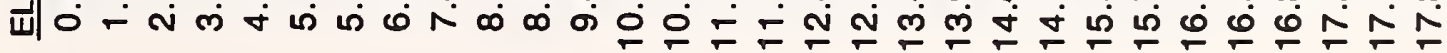

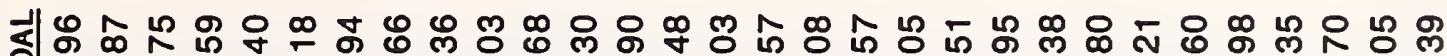
Oلర

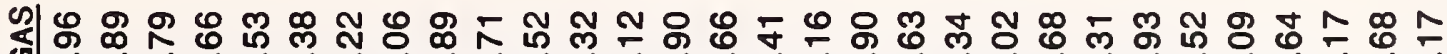
বद

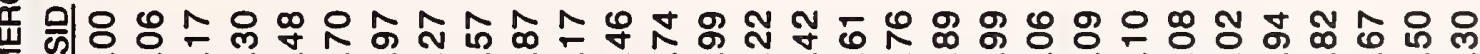
$\sum$ 嵌 ठ

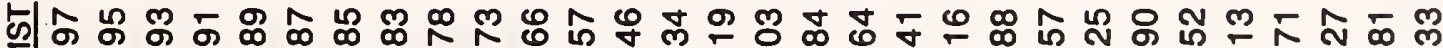
||-

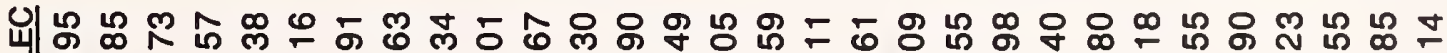
Ш|山

위요 ® 気

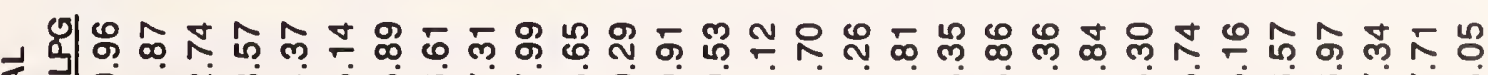
点

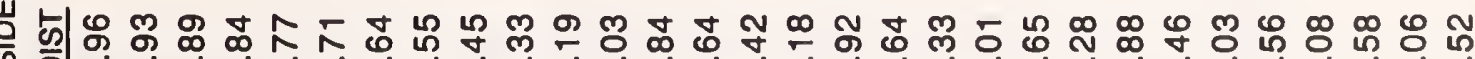
㟧

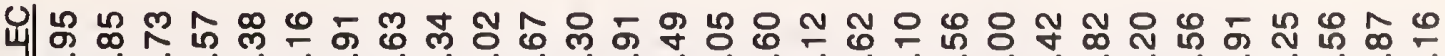
Шا山 0 -

zl- 


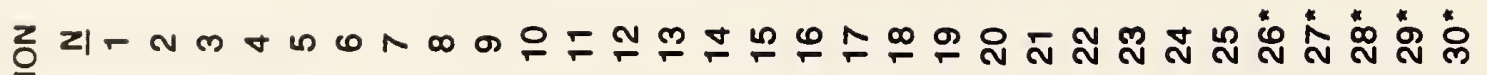

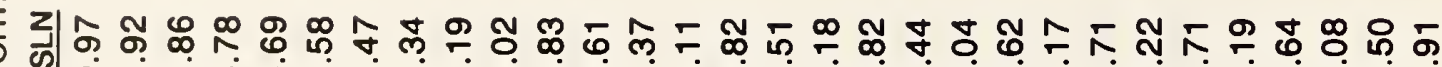

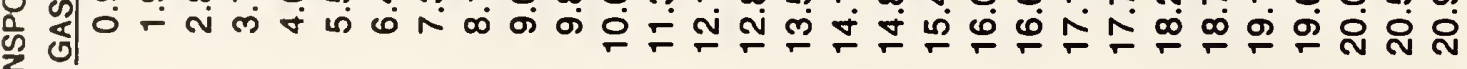
岳

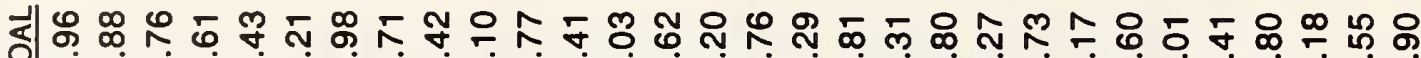

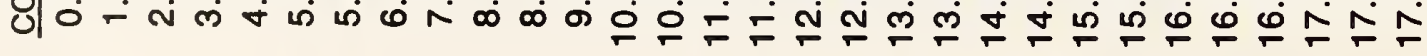

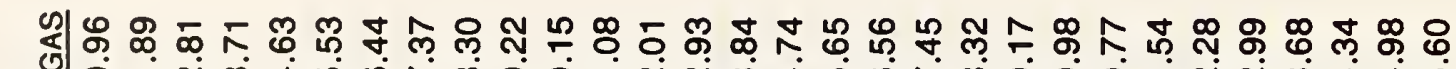
$\stackrel{1}{\frac{1}{\sigma}}$

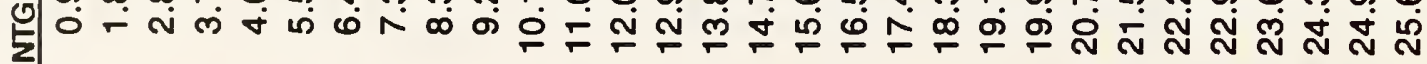

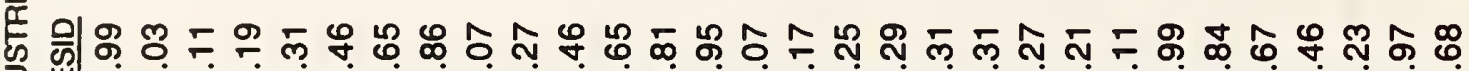
全嵌 ตㄴ. ప 刨命

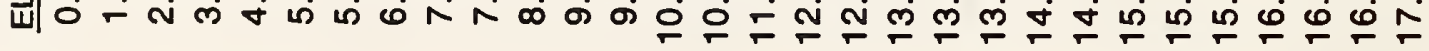

矛禹 ठإO-

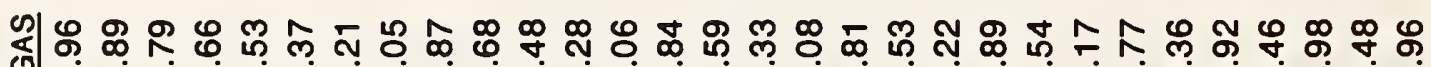

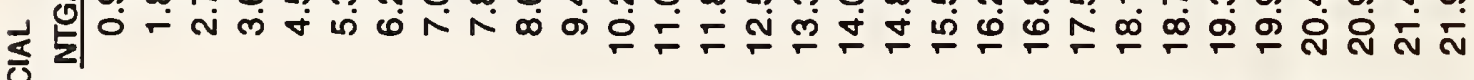
응

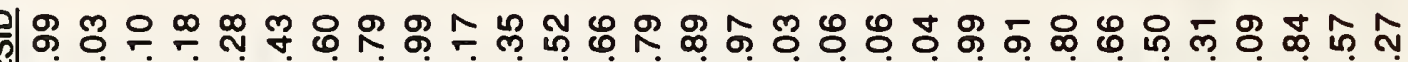

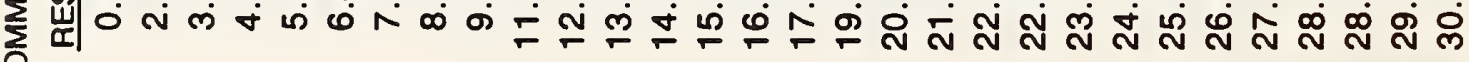
ช

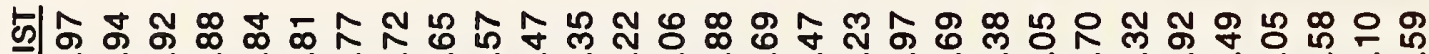
|)

讪 刑穴

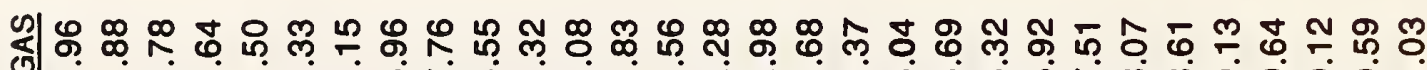
年

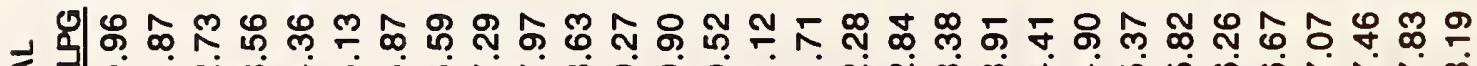
可 $\sum$

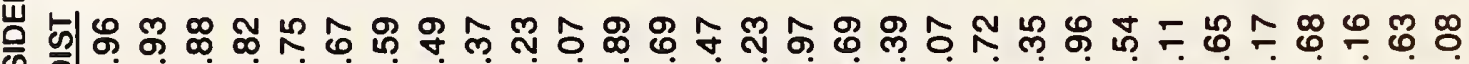
崖元宁

屾 崎

z| 


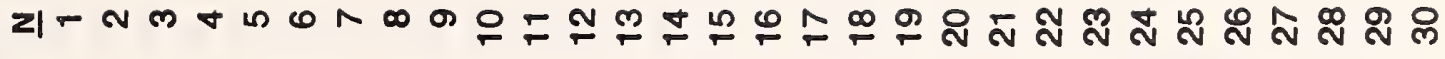

了ำ |

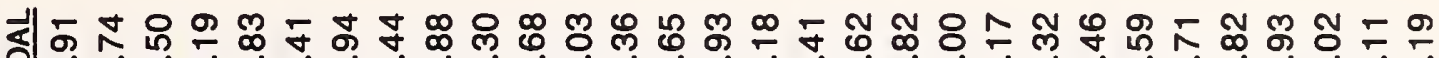

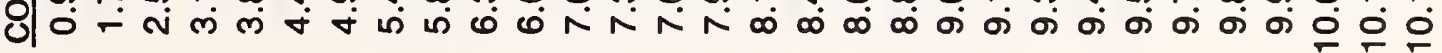

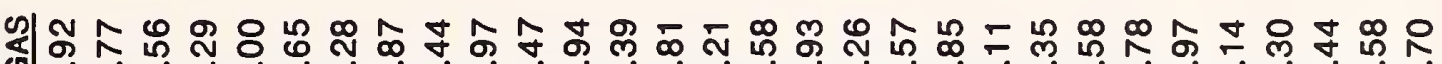
ही

结 嵌

능

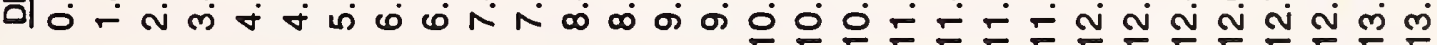

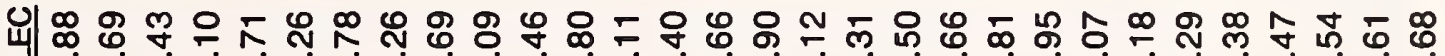
चان -

பीढ

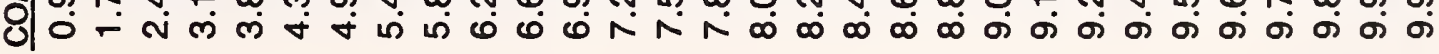

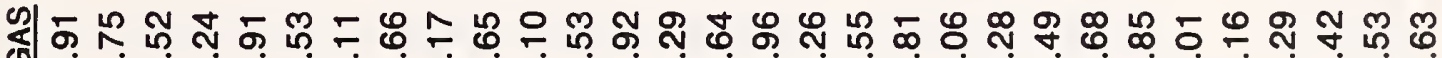
步

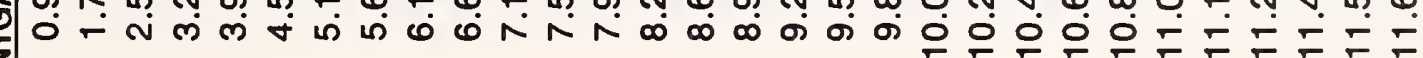

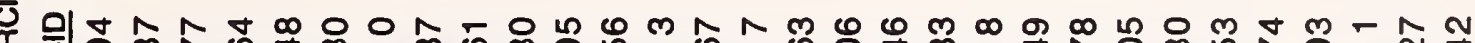
क

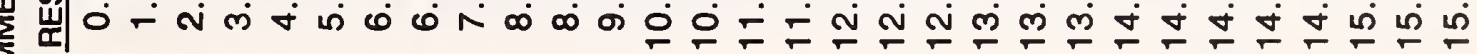

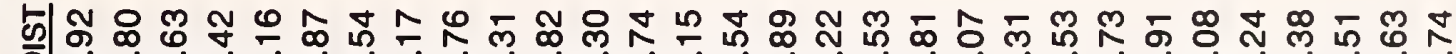
D|

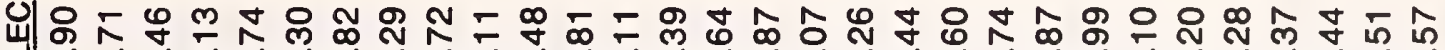
चأ 0 -

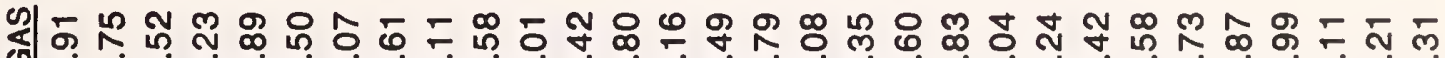
है - N ल लं

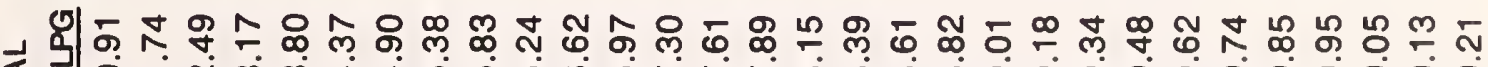

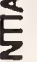

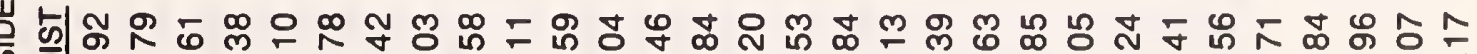
岸

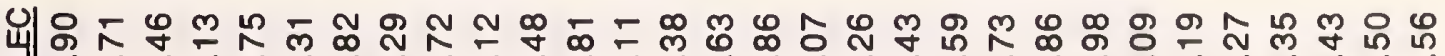

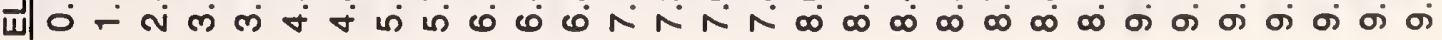

zा 
Z1-Nmナแ

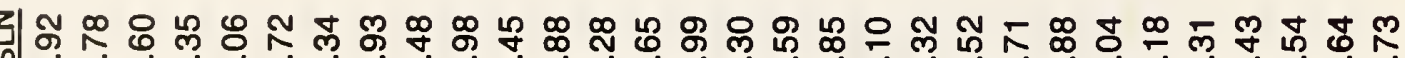
宁

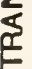

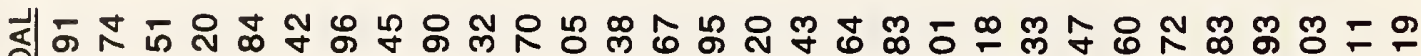
ठأ-

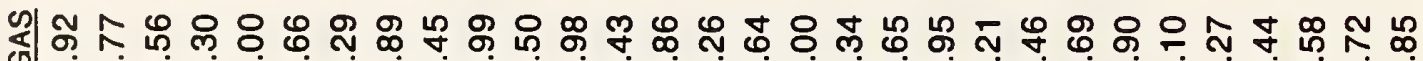

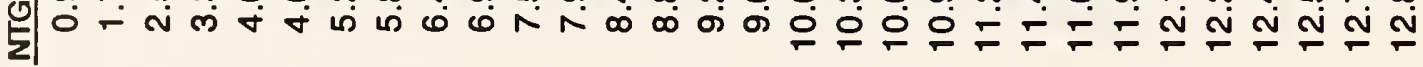
离

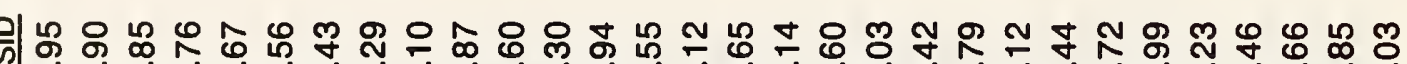

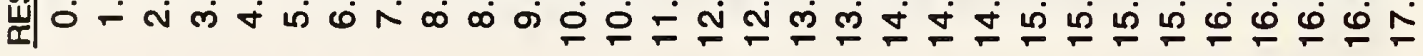

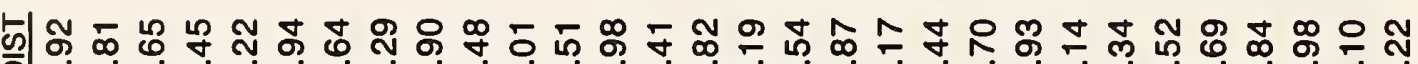
|ن

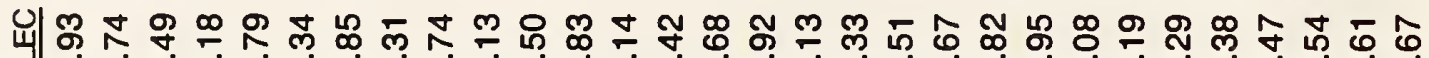

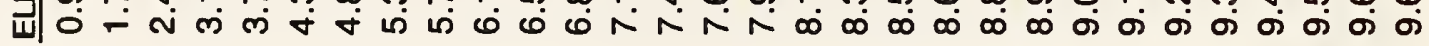

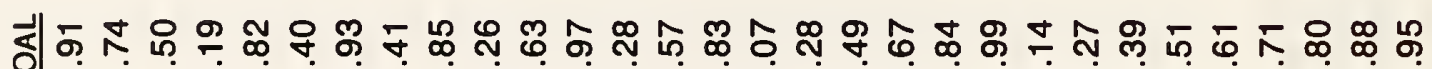

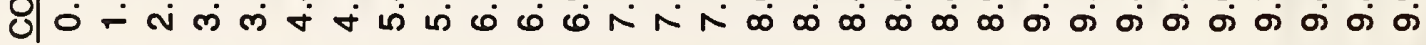

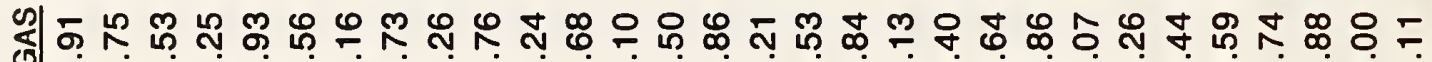
$\underset{5}{5}$ $\dot{0}-$ i ल

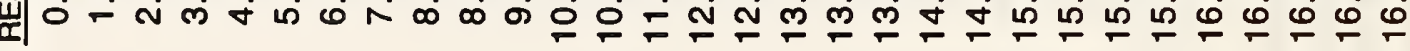

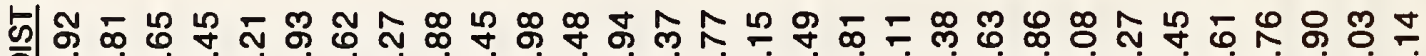

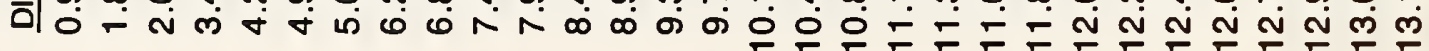

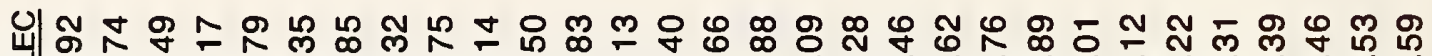

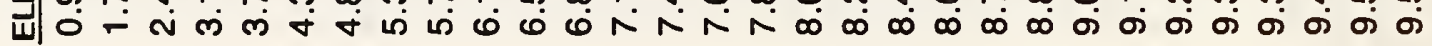

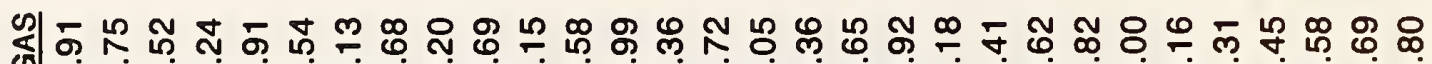

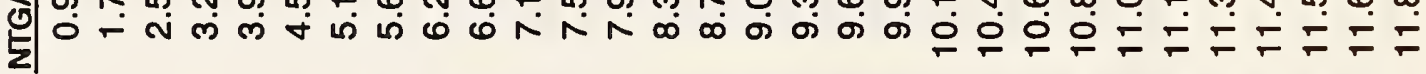

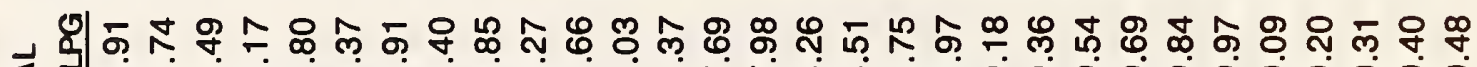

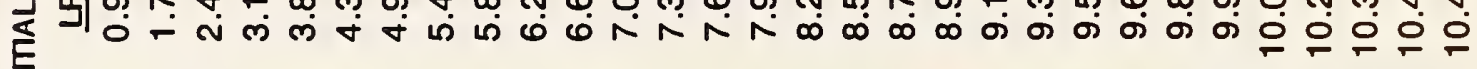

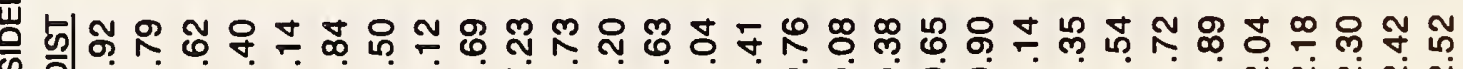
岩

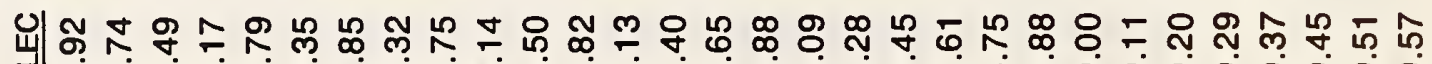

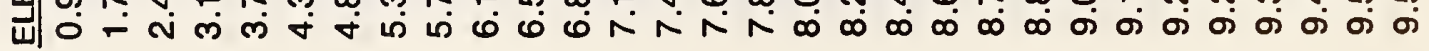

z|NM 
ㅣㅣ

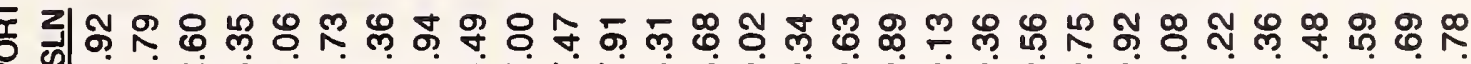
के ठ।

吝

뒤ำ ठإO

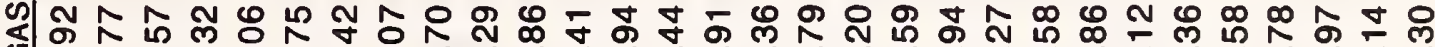
है

윔요

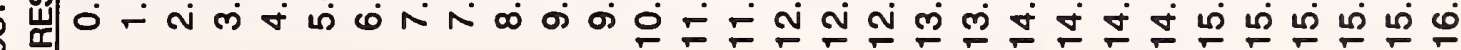

๒

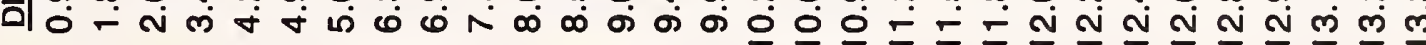

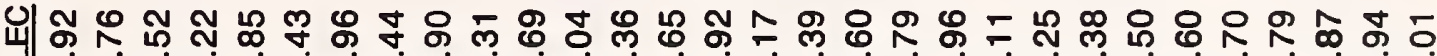
च| 0 -

ไป็

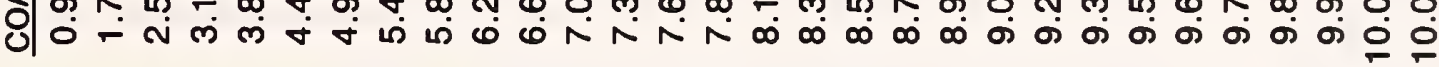

ๆ⿻ำ ○一

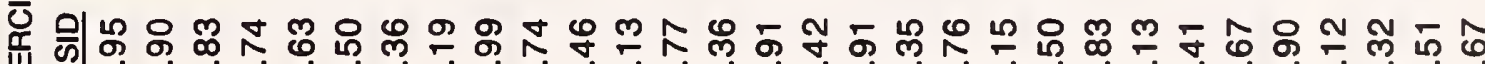
崩岸

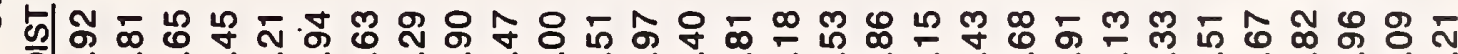
D|

岖 च) -

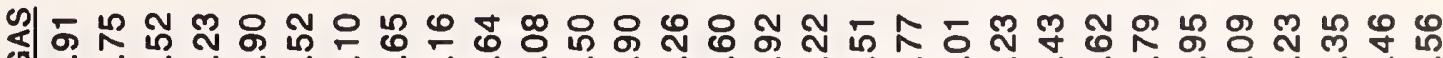
苟 m

幽 E -

岂 음 岸 0 -

馷 ш) - रल लं

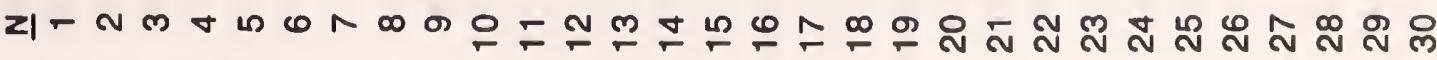




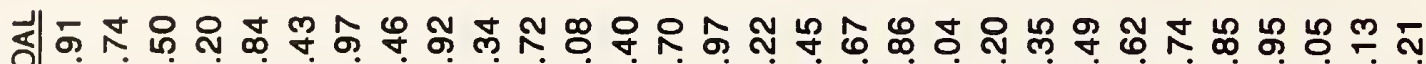

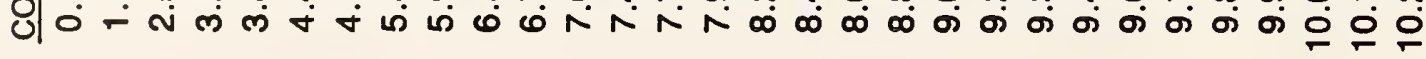

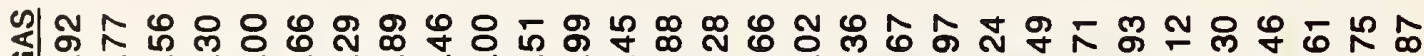
勿 $\vec{c}$

잉 顸 $0-$ i m

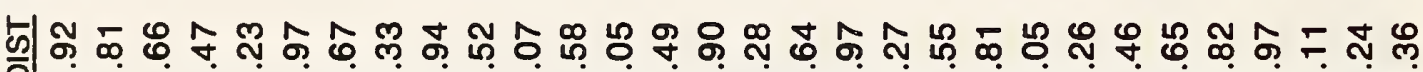

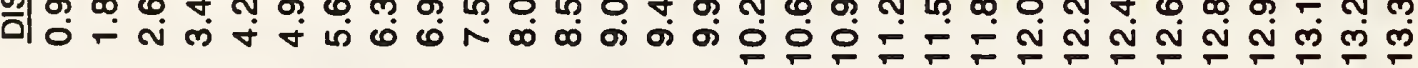

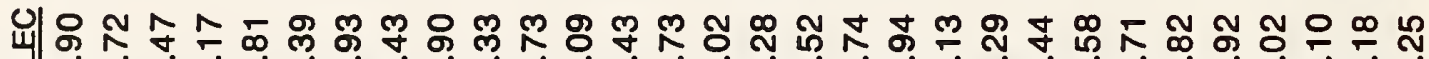

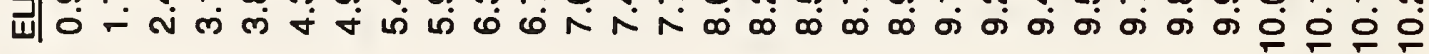

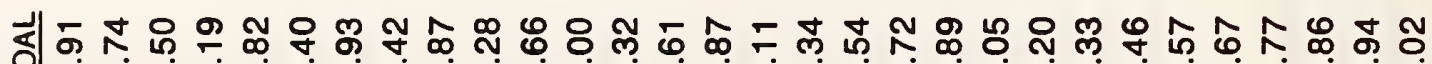
ठ우

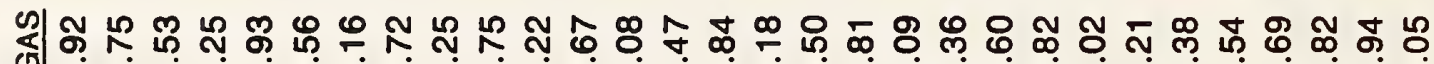
$+\frac{1}{2}$ 舟

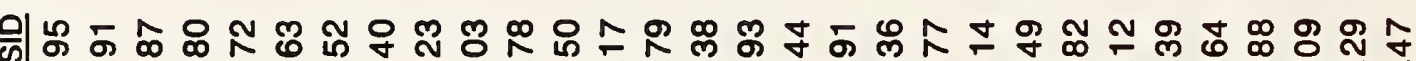
峲0 రํำ 更

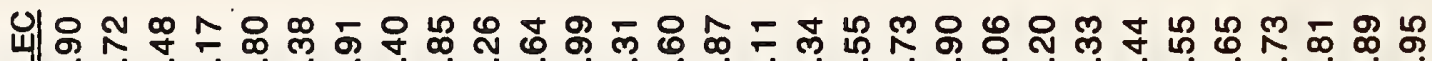
ची 0 -

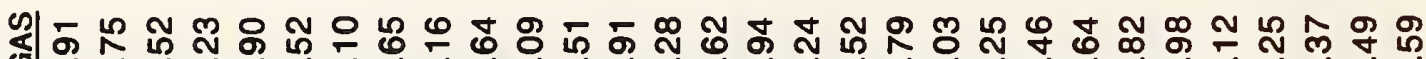
ह

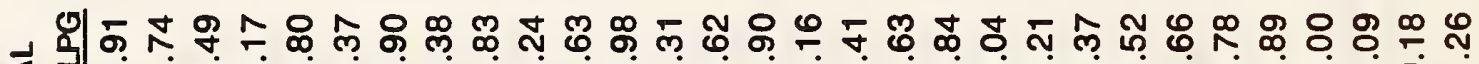
可 幽

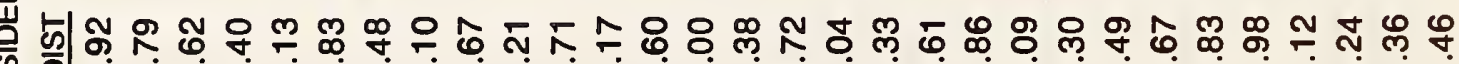
㞸

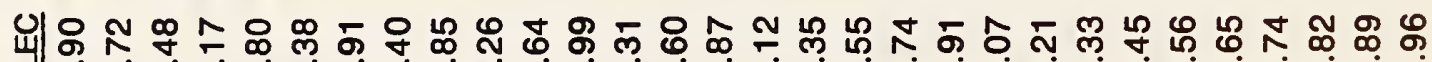
च)

Z|⿰亻丨 


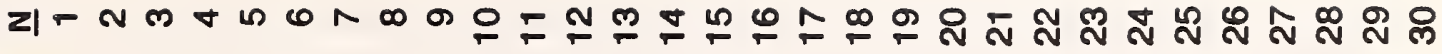
趁

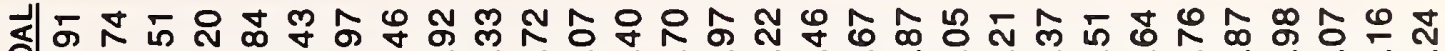

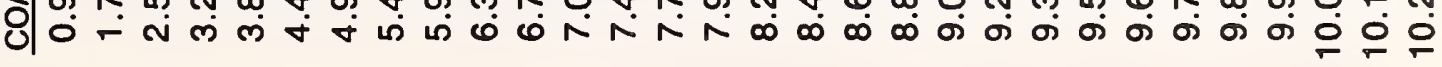

ํㅟㅎํ (j)

중

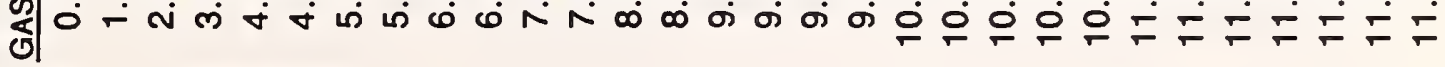

읶 岃|

⿹勹亾 б|

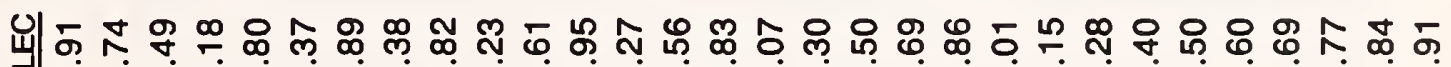

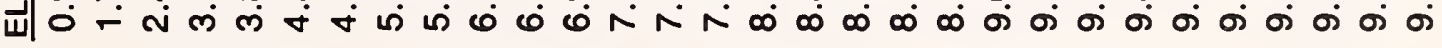

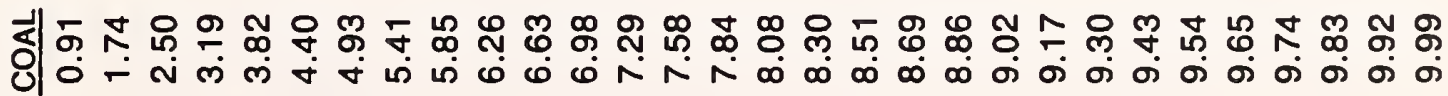

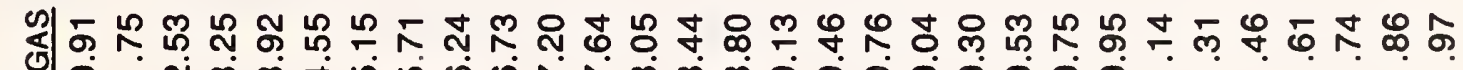

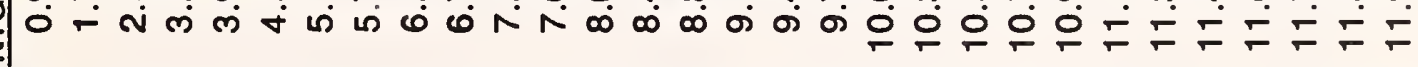

选

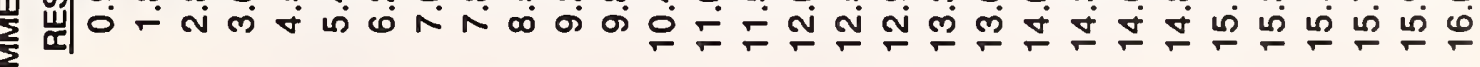

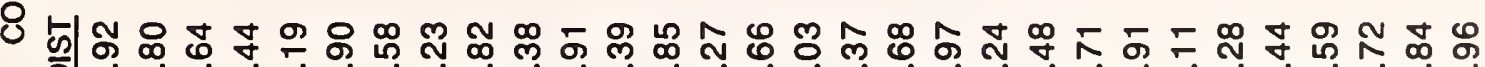

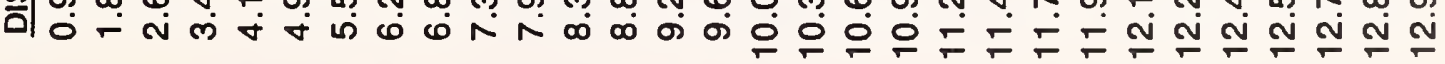

屾

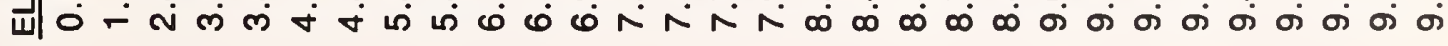

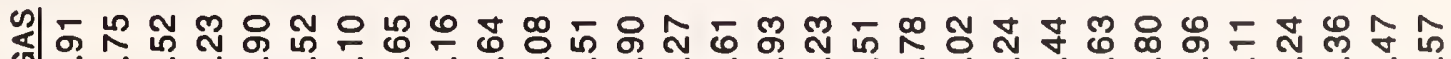

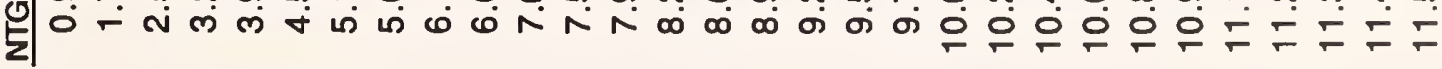
㲘市舟 列 $\sum_{\text {而 }}$

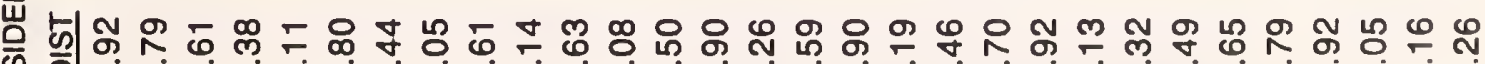
㞸

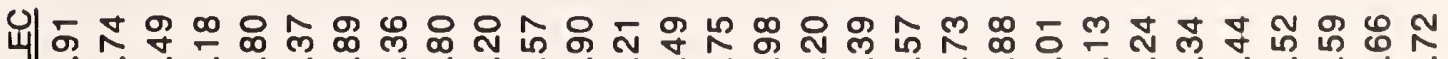

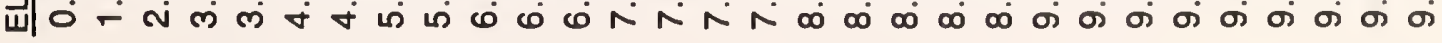

zा-N 
Projected average fuel price indices and escalation rates for Federal use (Indices and escalation rates exclude general price inflation)

Tables $\mathrm{Ca}-1$ through $\mathrm{Ca}-5$ present projected average fuel price indices for the 4 Census regions and for the United States. These are multipliers which when applied to 1991 prices provide estimates of the corresponding future-year prices in 1991 dollars. Note that the resulting price estimates are in constant dollars, exclusive of general price inflation. Constant dollar prices are needed when discounting is performed with discount rates which do not include general price inflation.

Example of How to Use the Indices:

To estimate the price of industrial steam coal in year 2005 in Connecticut, go to table $\mathrm{Ca}-1$, find the year 2005 index for industrial steam coal (1.13), and multiply by the 1991 price for industrial steam coal in Connecticut. The result is in 1991 dollars.

Tables $\mathrm{Cb}-1$ through $\mathrm{Cb}-5$ present the projected average fuel price escalation rates (percentage change compounded annually) for seven selected periods from 1991 to 2021 for the 4 Census regions and for the United States. Note that these are "real" rates exclusive of general price inflation. Their use results in prices expressed in constant dollars.

The escalation rates consolidate the information provided by the indices in the $\mathrm{Ca}$ tables so that trends in projected price changes can be seen at a glance. They are provided primarily to accommodate those who use computer programs which require escalation rates as inputs.

Unless there is a compelling reason to use escalation rates, it is recommended that you use the indices in the $\mathrm{Ca}$ tables when you need estimates of future-year energy prices, since the indices include year-to-year information rather than averages over a number of years.

\section{Example of How to Use the Escalation Rates:}

To estimate the price of residential distillate in 1998 ( $\mathrm{P}_{98}$ ) in Wyoming using the escalation rates, go to table $\mathrm{Cb}-4$ and find the 1991-1995 and 1995-2000 escalation rates for residential distillate ( 3.268 and 3.468 per year, respectively). Enter these values and the actual 1991 price of residential distillate in Wyoming $\left(\mathrm{P}_{9_{1}}\right)$ into the following formula. Then solve for the 1998 energy price (stated in 1991 dollars):

$$
\begin{aligned}
P_{98} & =p_{91} \times\left(1+e_{1}\right)_{1}^{k} \times\left(1+e_{2}\right)_{2}^{k}, \\
& =p_{91} \times(1+0.0326)^{4} \times(1+0.0346)^{3}
\end{aligned}
$$

where $e_{i}=$ Annual compound escalation rate for period $i$ from the $\mathrm{Cb}$ tables (in decimal form); and

$k_{1}=$ Number of years over which escalation rate $e_{1}$ occurs. 
For further explanation of how to use these tables, see NIST Handbook 135.

Note: The data in the tables which follow are reported by 4 Census regions . Figure B-1 presents a map showing the states corresponding to the 4 Census regions. The Census regions do not include American Samoa, Canal Zone, Guam, Puerto Rico, Trust Territory of the Pacific Islands, or the Virgin Islands. Analysts of Federal projects in these areas should use data which are "reasonable under the circumstances," and may refer to the tables with U.S. average data for guidance. 


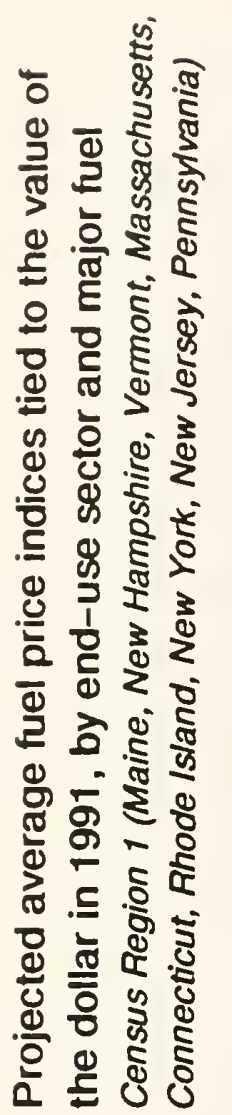

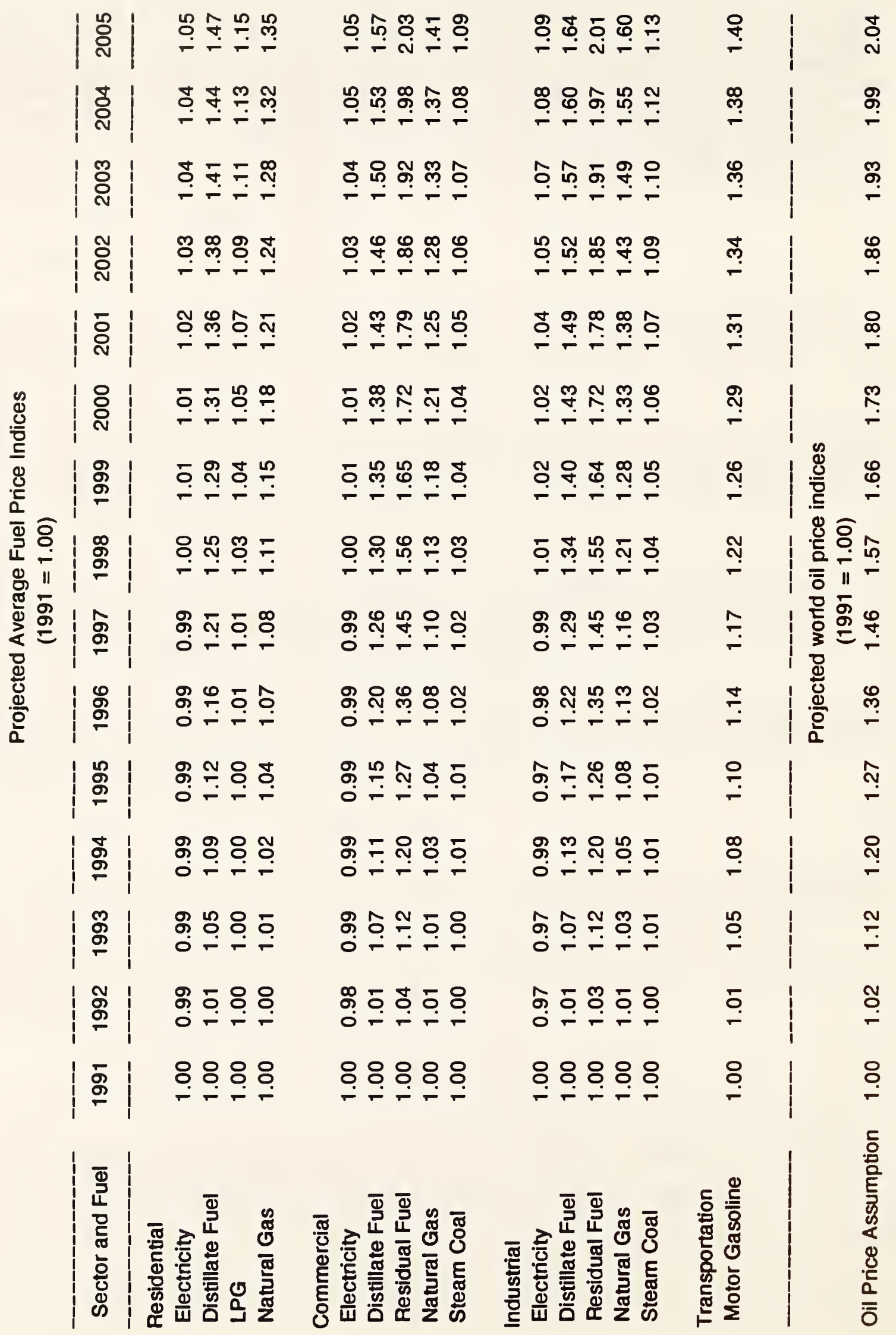




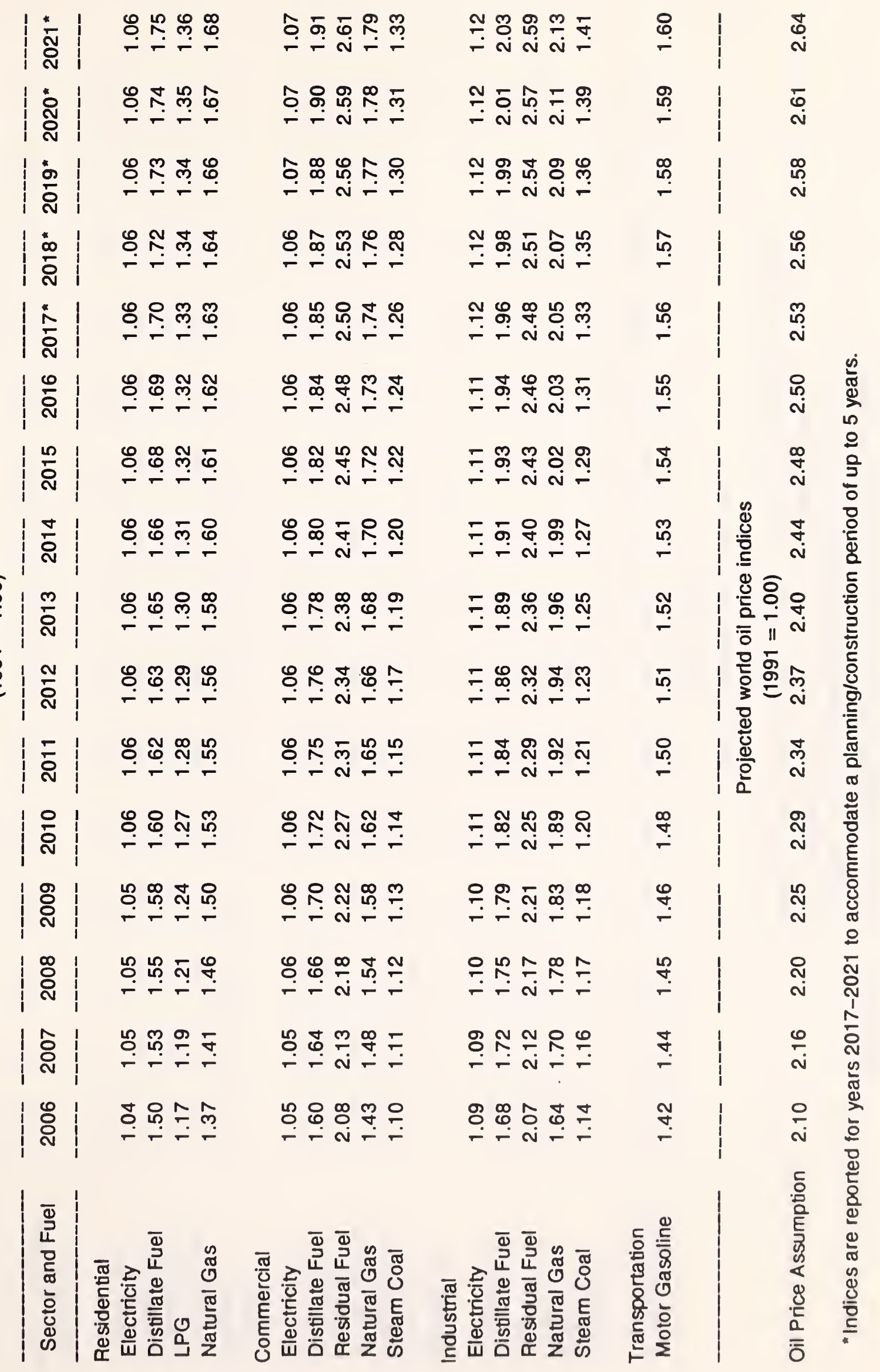




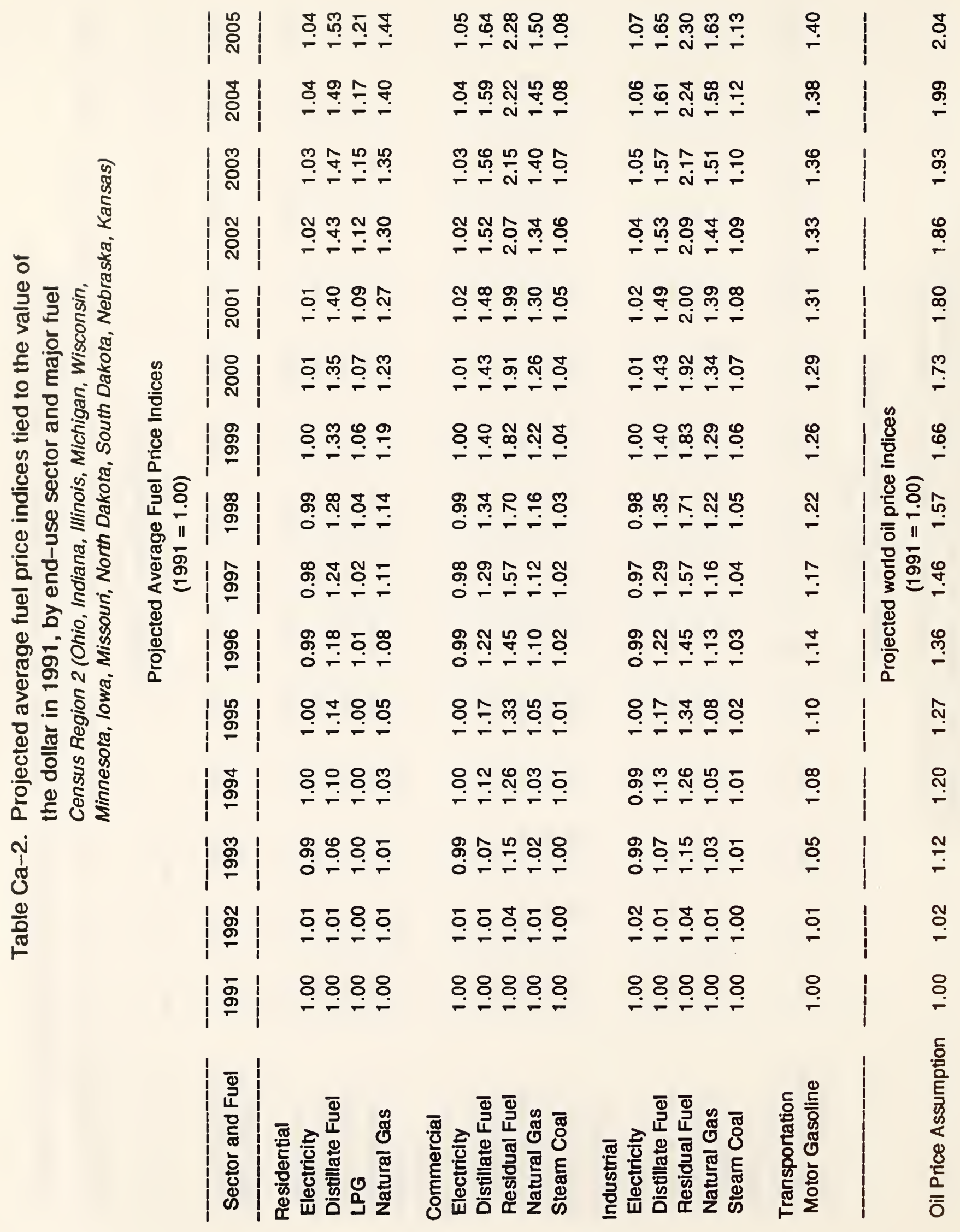




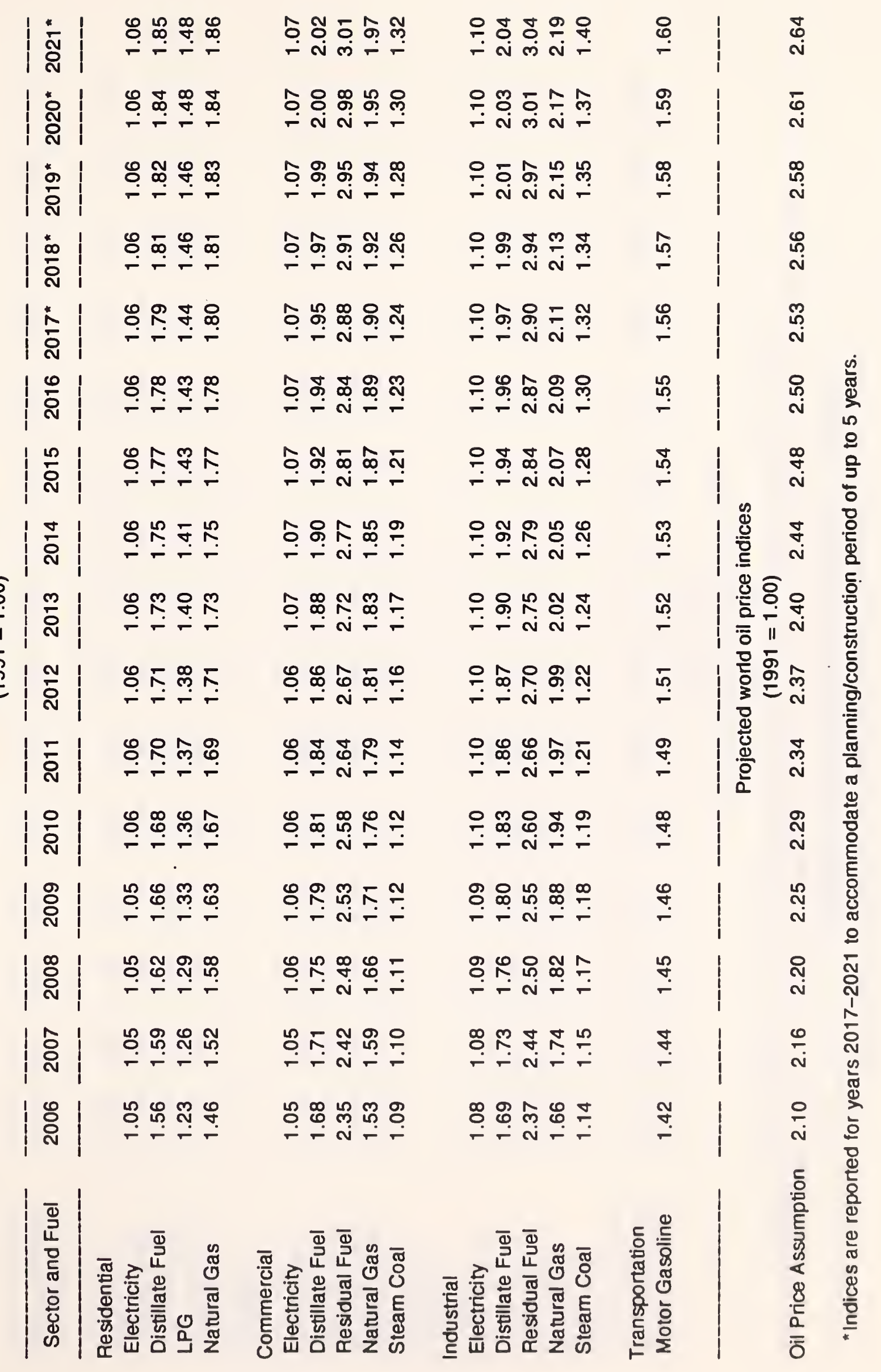




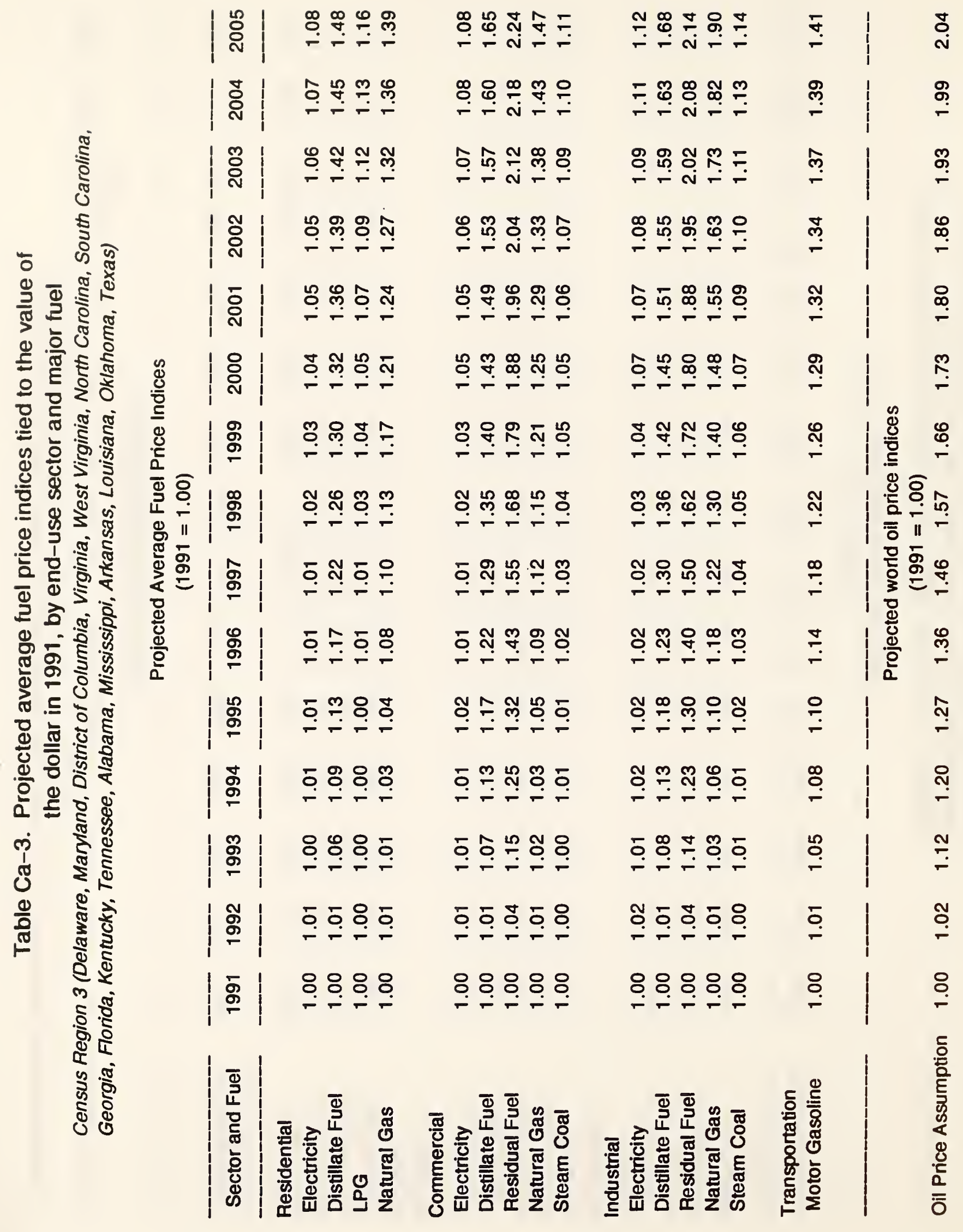




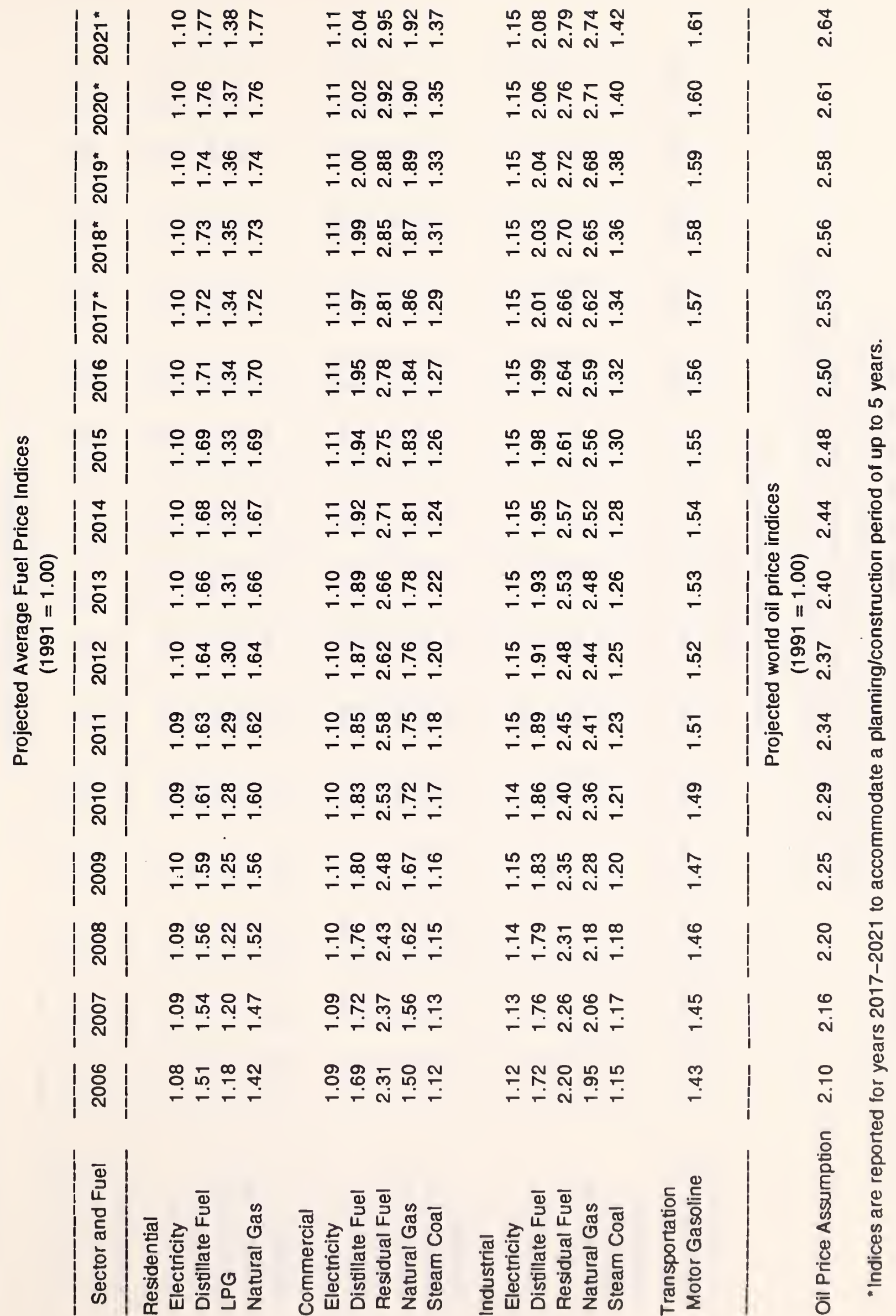




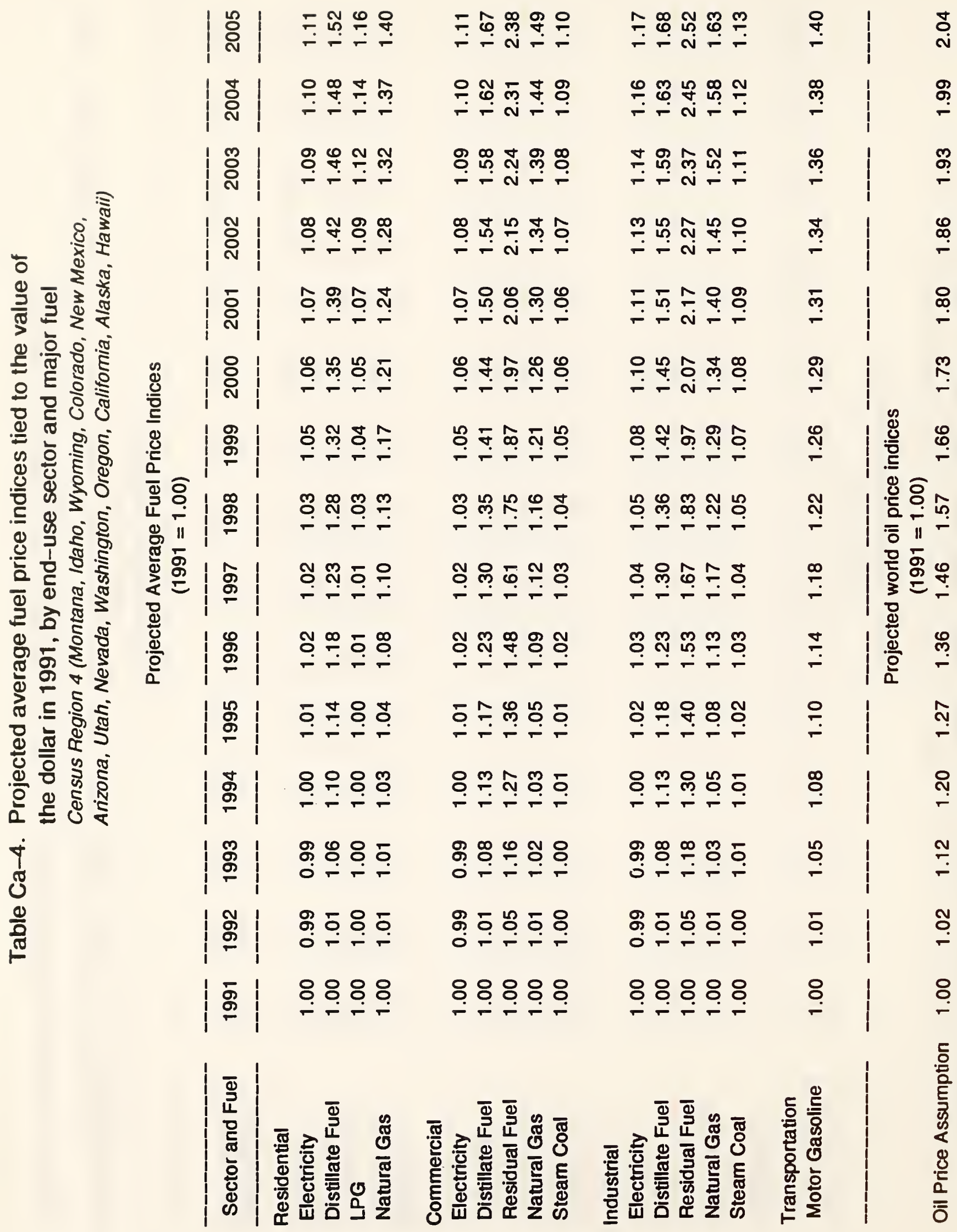




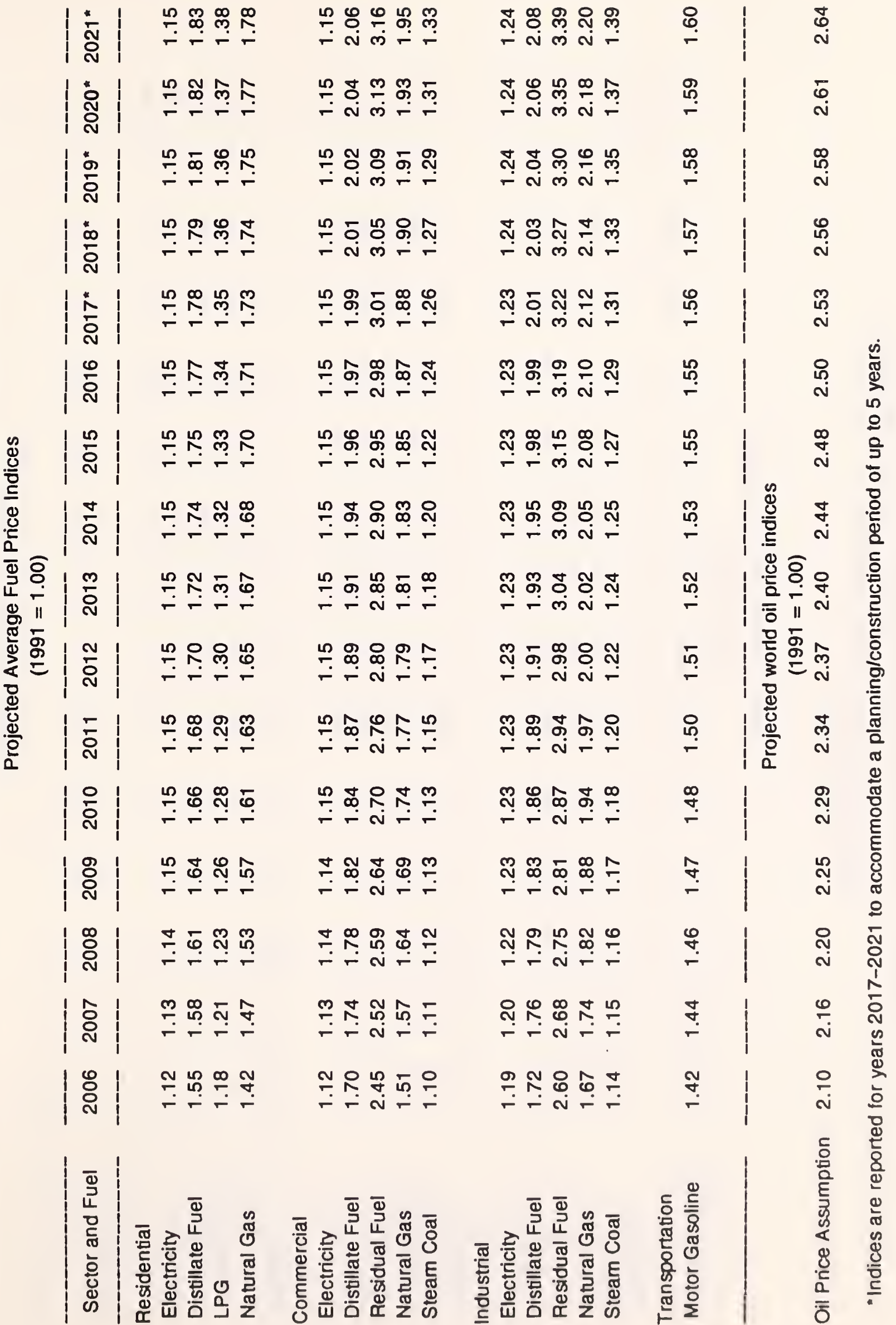


"

$\stackrel{\frac{1}{3}}{\frac{2}{3}}$

高

.5

\&

을 응

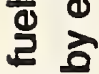

迹宫

ए

.

这

$\frac{\Phi}{0}$ 을

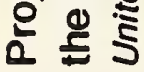

0
1
0
0
0
0
$\frac{0}{0}$

|

|

|

|

|

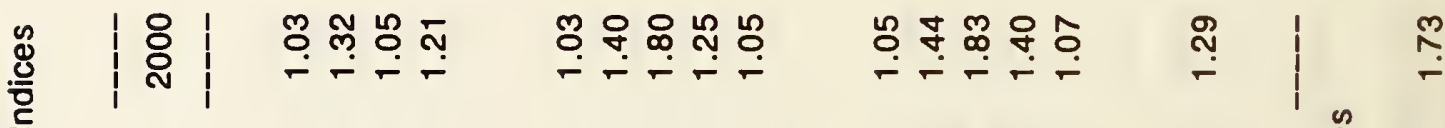

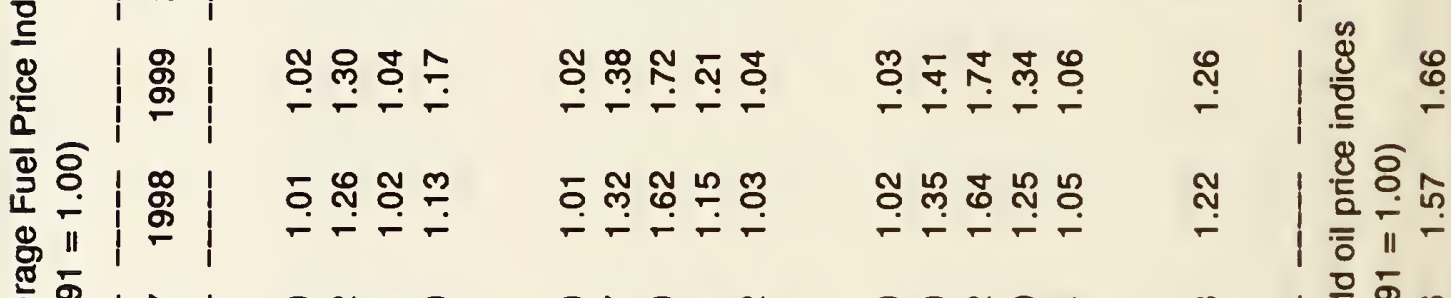

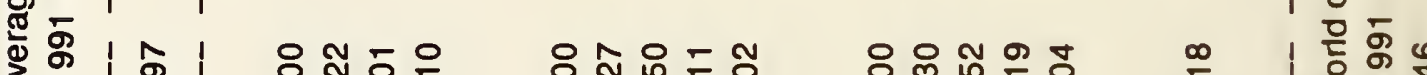

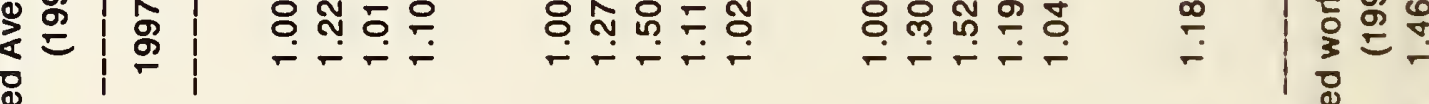

$\frac{\frac{\pi}{0}}{\frac{0}{2}}$

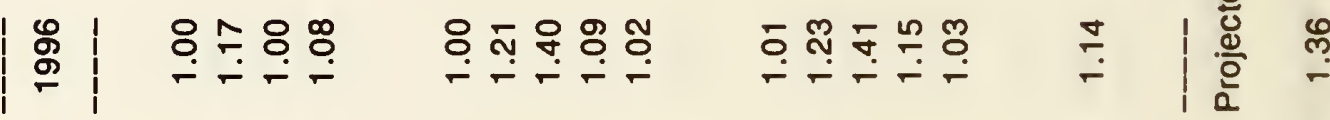

各|

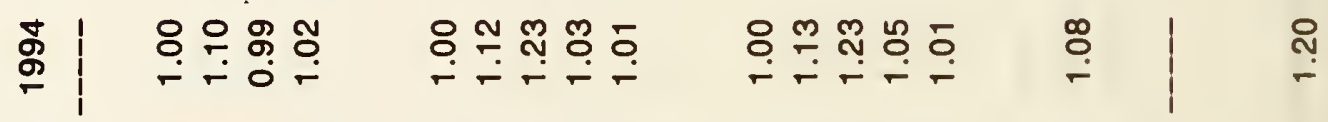

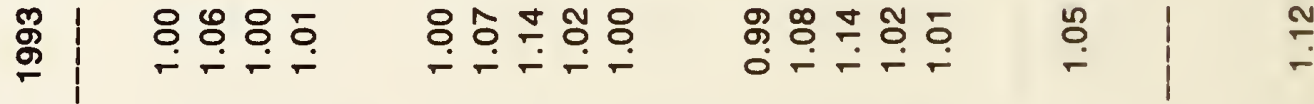

|

|

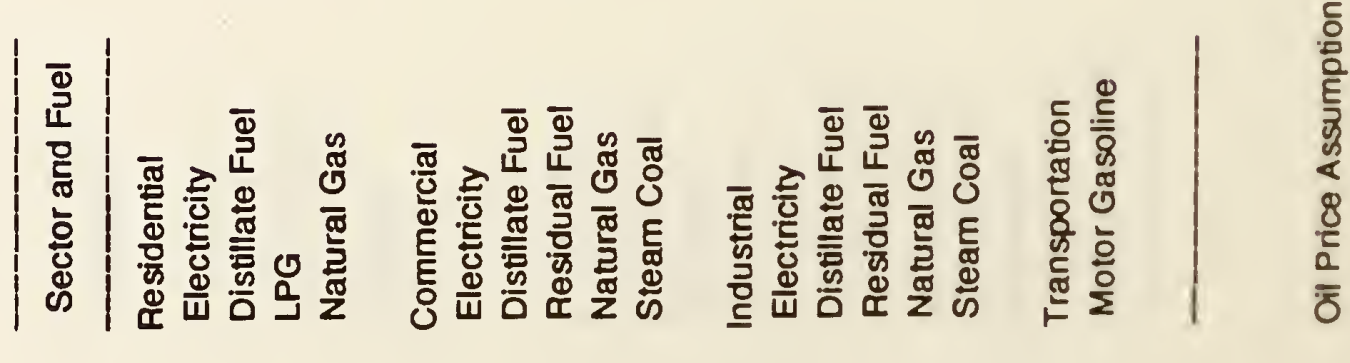




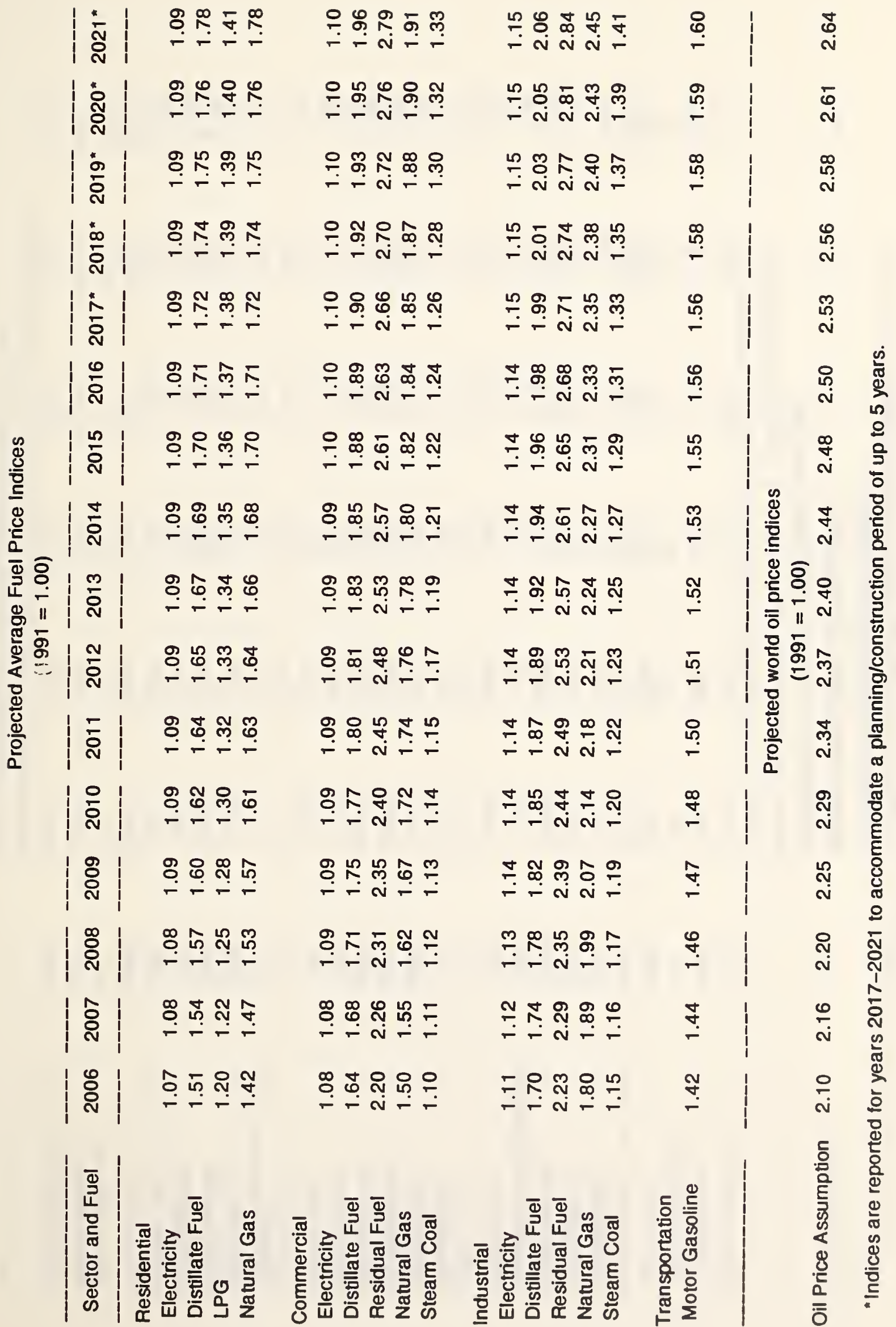




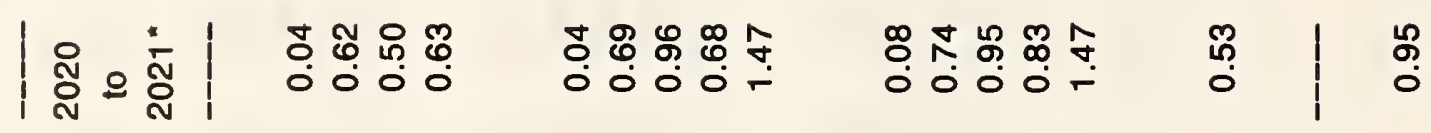

忽 |

호

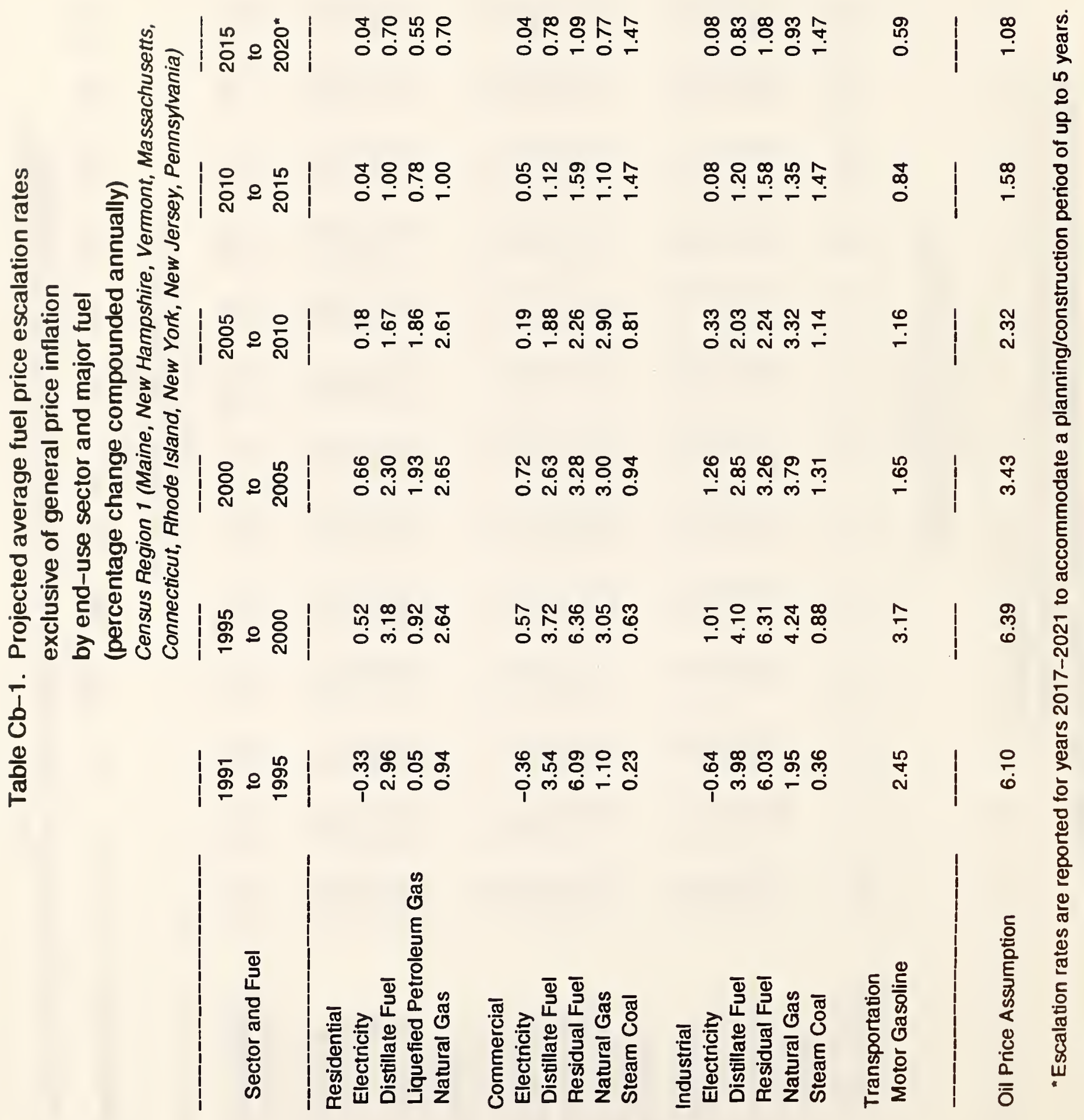




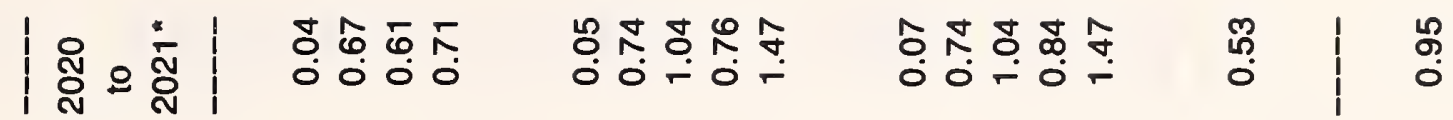

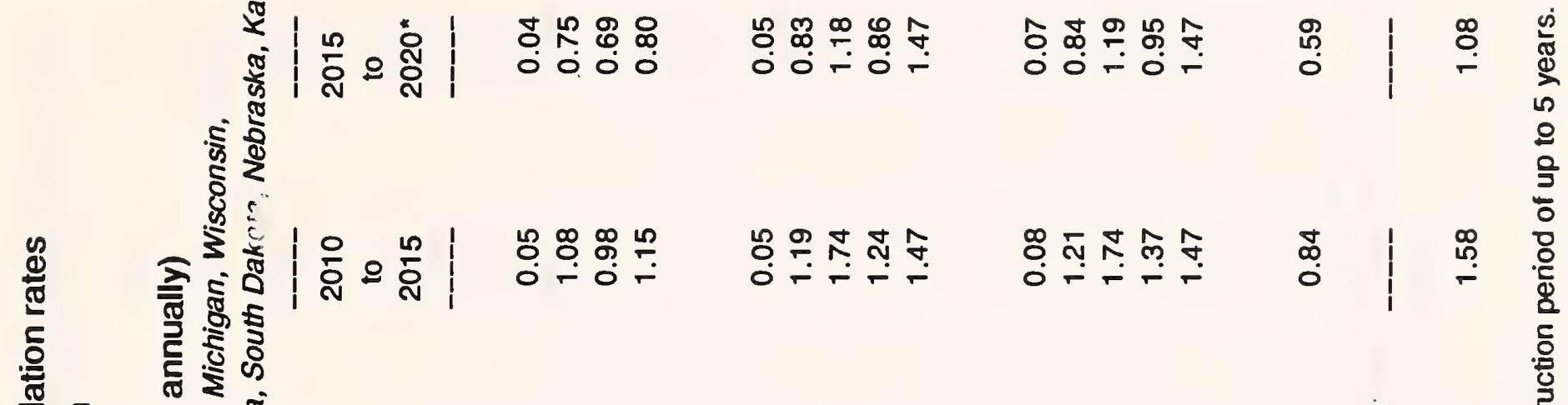

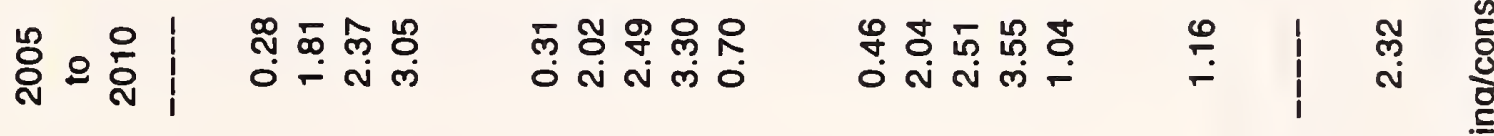

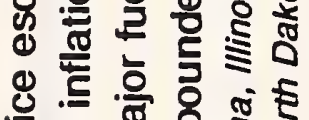

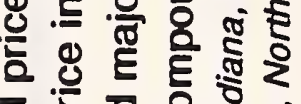

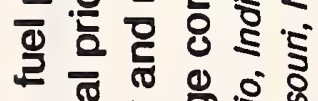

๑ั

สิ

¿

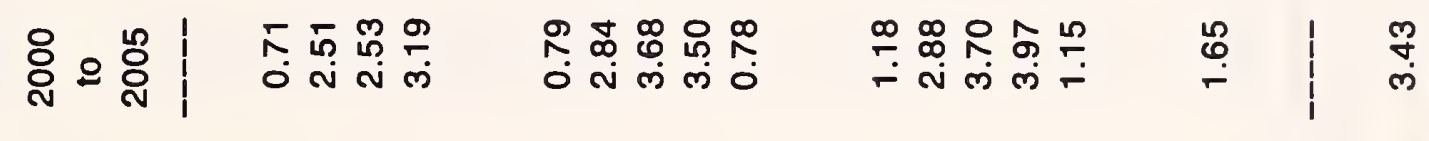

ग

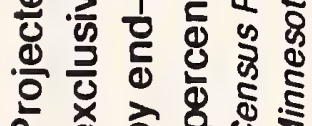

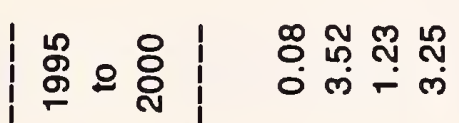

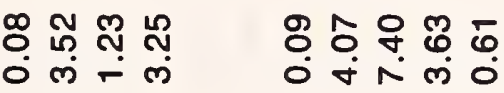

mำ

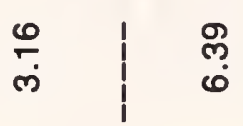

N

耑

$\frac{0}{2}$

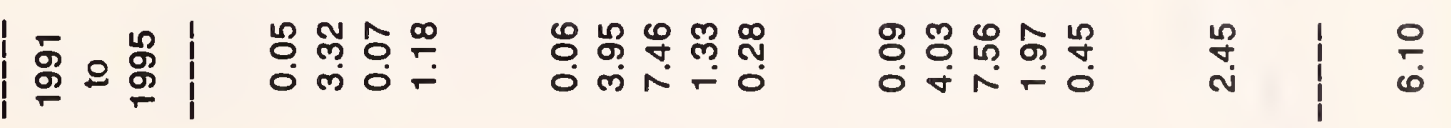

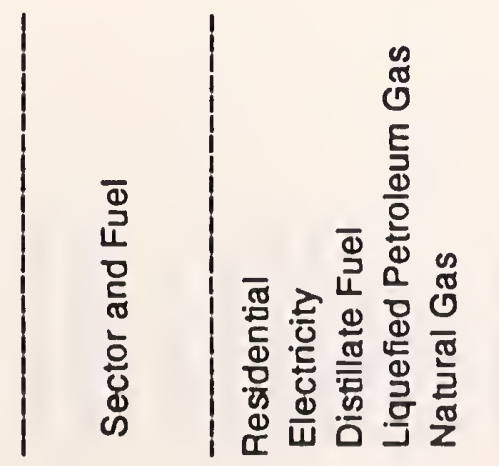

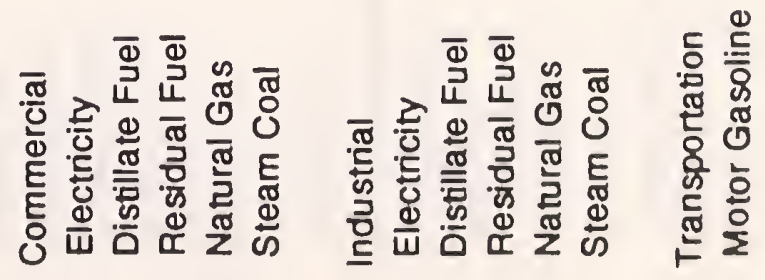

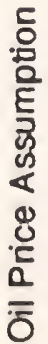

을

은

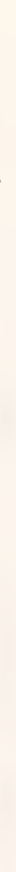




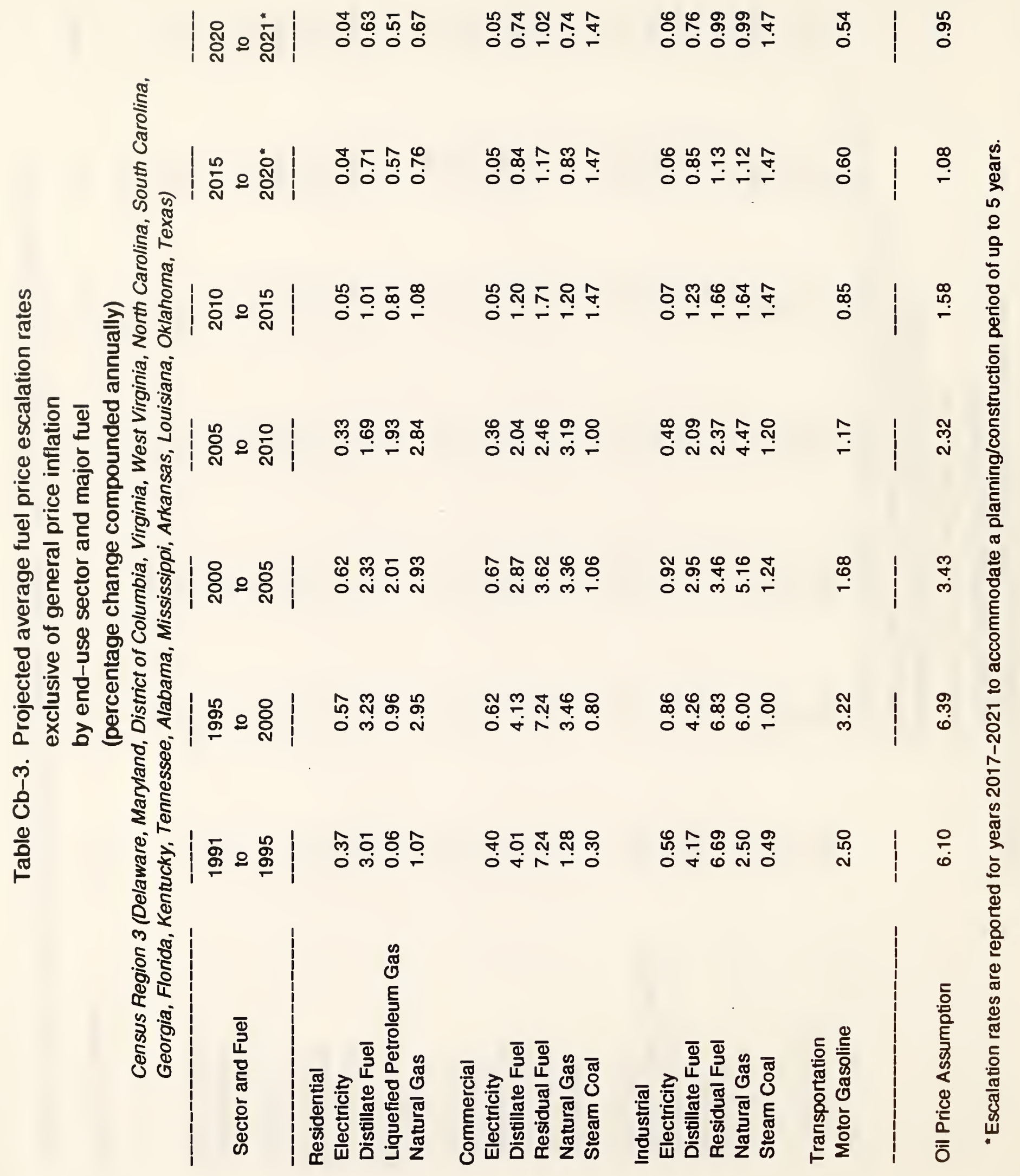




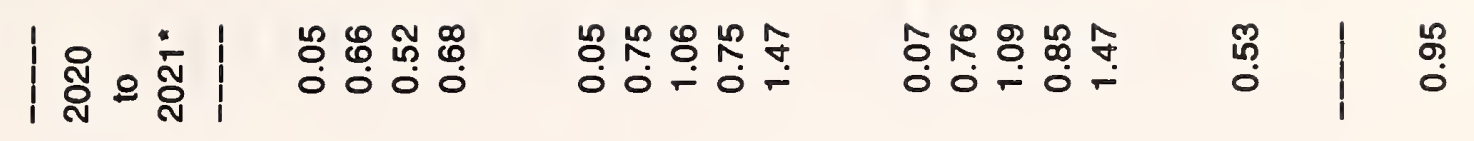

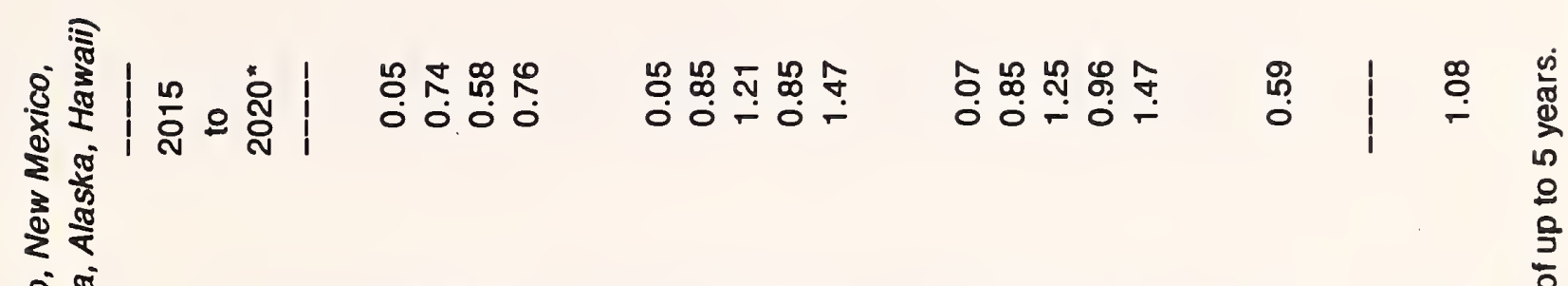

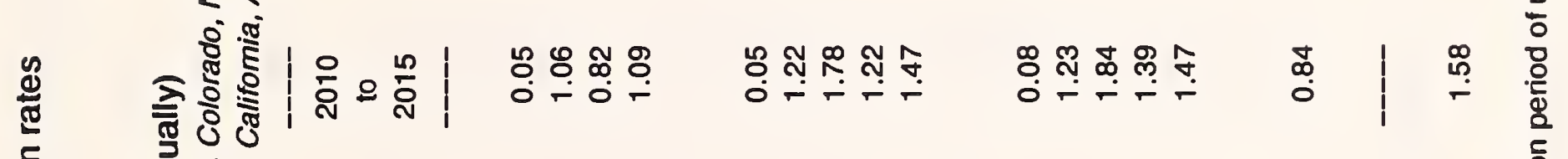

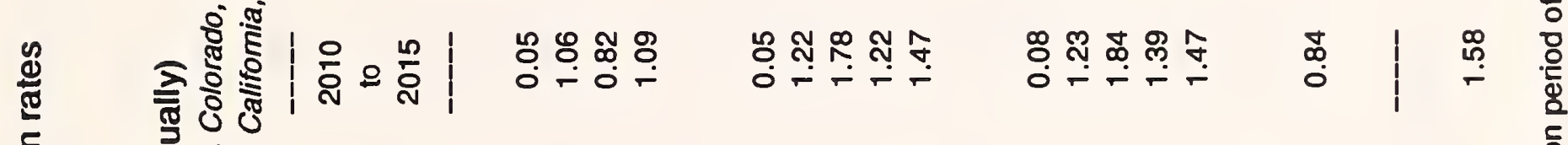

结

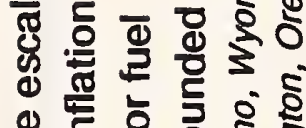

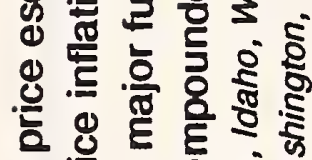

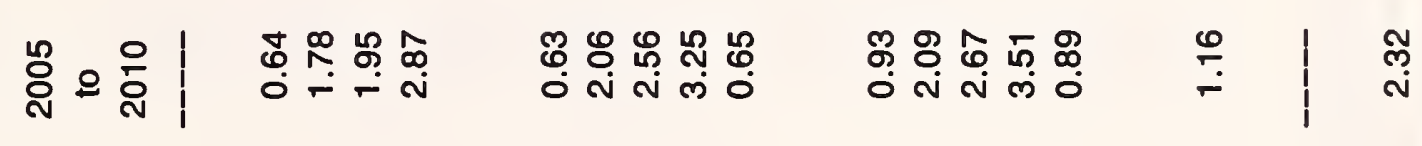

ฮ 을 은 ญ

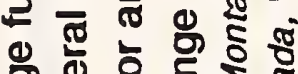

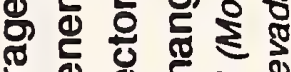

๘

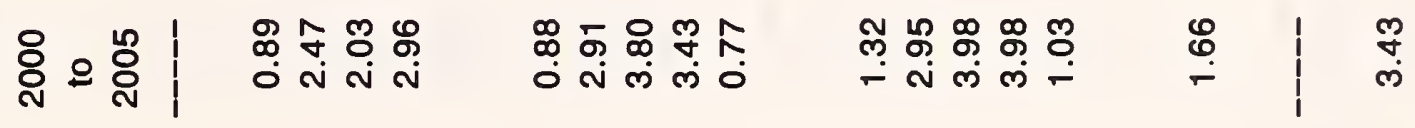

สัธ

○

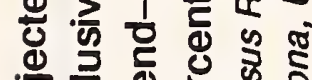

응 Ð

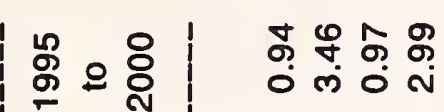

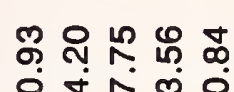

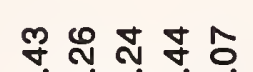

$\stackrel{\infty}{\dot{m}}$

$\dot{1}$
$\frac{0}{0}$
$\frac{0}{0}$
$\frac{0}{\mathscr{N}}$

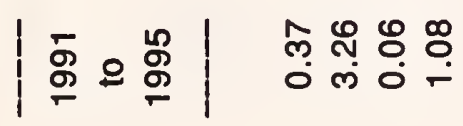

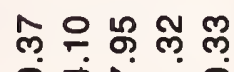

ผกำ

\begin{tabular}{l|l}
$\stackrel{0}{\sim}$ & $\frac{0}{6}$
\end{tabular}

$\therefore \stackrel{2}{2}$

०ं

$0 \forall \infty \div 0$
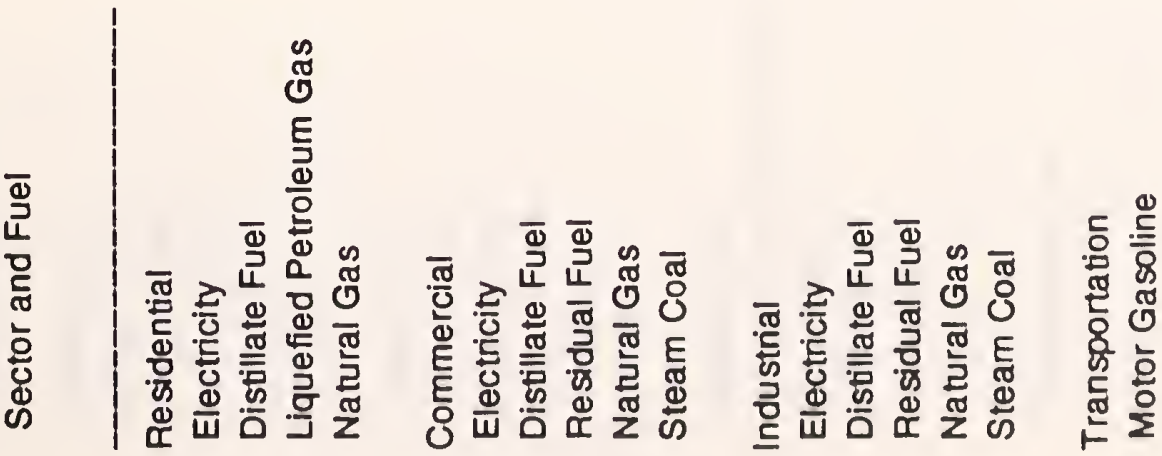


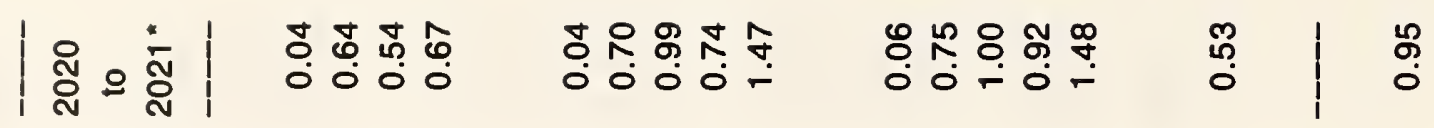

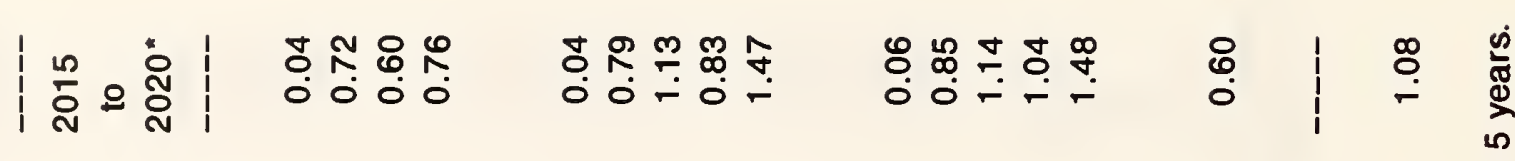

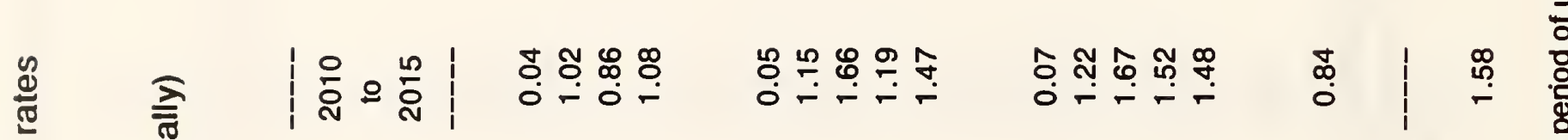

产 章

要

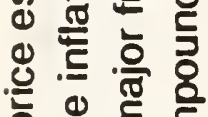

|

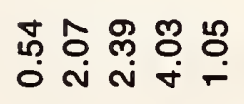

\begin{tabular}{l|l}
- & $N$ \\
- & ले
\end{tabular}

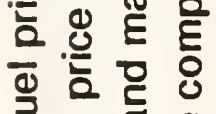

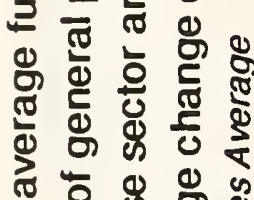

तo 0 \%

证

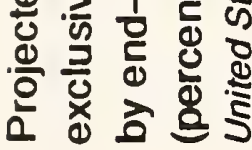

$\left|\begin{array}{ll}0 & 0 \\ \Omega & 8 \\ N\end{array}\right|$

:

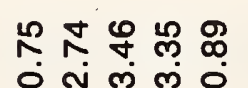

三尺ْำ

$\stackrel{8}{-}+\frac{7}{\infty}$

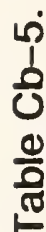

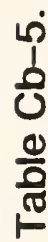

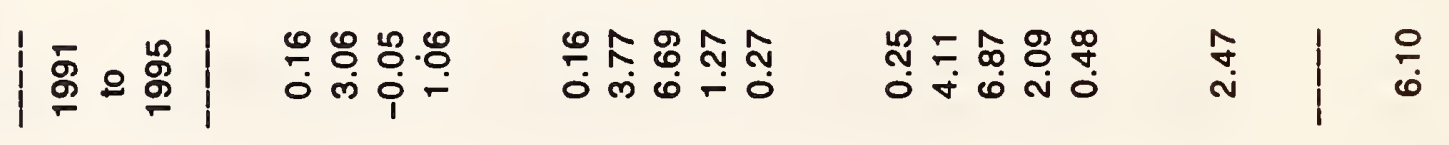

|

.

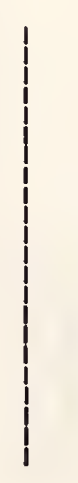

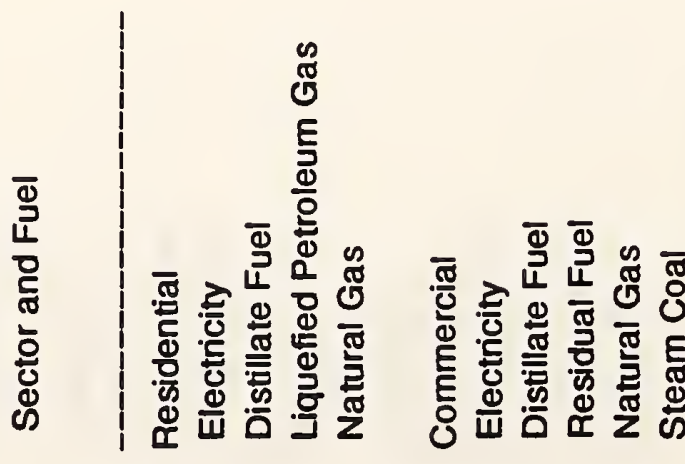
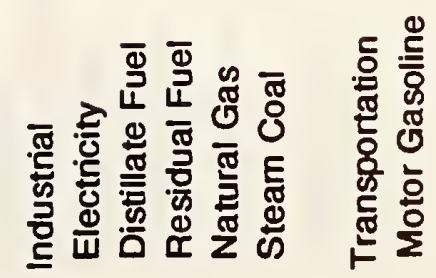

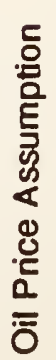


Factors for updating appliance label values in compliance with the Energy Conservation Mandatory Performance Standards for New Federal Residential Buildings ( 10 CFR 435)

Compliance with energy conservation performance standards for new Federal residential buildings requires calculation of a building's energy costs, including appliance costs. For this purpose, label values for gas and electric water heaters are given in the Federal micro-computer program, COSTSAFR, as $\$ 176$ and $\$ 406$ per year, respectively, in 1987 dollars, and for refrigerator/freezers, as $\$ 61$ per year in 1987 dollars. To adjust 1987 prices to 1991 prices, multiply these 1987 label values by the factors below.

Table D. Factors for updating Appliance Label Values

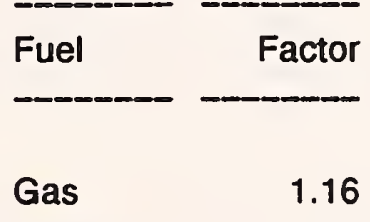

Electricity $\quad 1.11$ 
Projected average fuel price indices for private sector use (Indices are given inclusive of four alternative rates of general price inflation)

This section presents in tables S-1 through S-5 projected average fuel price indices for 4 fuels in the residential sector and 5 fuels in the commercial sector for each of the years from 1991 through 2021. They update tables originally published in the report, Comprehensive Guide for Least-Cost Energy Decisions (NBS SP 709).

As a convenience for the user, the indices include the effect of 4 alternative, hypothetical rates of general price inflation: $0,2.5,5$, and 7.5 percent. Selection of these rates is intended in no way to suggest what actual rates might be. Use of the indices produce price estimates which are in current dollars, inclusive of general price inflation. Current dollar prices are needed when discounting is performed with discount rates which include general price inflation. For the case of 0 percent inflation, the price indices in the $S$ series of tables are identical to those in the counterpart $\mathrm{Ca}$ table series. When there is no inflation, there is no difference between constant and current dollars.

The indices based on inflation rates of $2.5,5$, and 7.5 percent allow the analyst to perform evaluations based on the assumption of a positive rate of general price inflation that changes the purchasing power of the dollar. Performing evaluations in current dollars is sometimes preferred for private investment decisions, primarily because it facilitates the treatment of taxes.

The indices in tables S-1 through S-5 are derived from the indices reported in tables $\mathrm{Ca}-1$ through $\mathrm{Ca}-5$ by means of the following equation:

$$
I_{S}=I_{C} \times(1+g)^{N} \text {, }
$$

where $I_{S}=$ index found in tables S-1 through S-5;

$I_{C}=$ index found in tables $\mathrm{Ca}-1$ through $\mathrm{Ca}-5$;

$g=$ annual rate of general price inflation in decimal form; and

$\mathrm{N}=$ number of periods, in this case equal to the year of the index minus 1991.

\section{Example of How to Use the Indices:}

Suppose you wish to estimate the present value of energy savings in year 2000 , and you expect an annual inflation rate of 5 percent per year. Taking natural gas for residential use in Maryland, estimate present value savings as follows: (1) multiply the 1991 price for residential natural gas in Maryland by the projected quantity of energy savings in the year 2000 , to estimate savings in the year 2000 in 1991 prices, (2) go to table S-3, find the year 2000 index for residential natural gas at a 5 percent inflation rate (1.87), 
and multiply the index by the result from (1) to determine savings in the year 2000 in 2000 prices, and (3) discount the savings back to the present, using an SPW factor based on a discount rate that reflects a 5 percent inflation rate. To obtain present value savings over the entire study period, this calculation must be repeated for each year that there are savings, and the results summed. (UPW factors are not given for private sector use because of the large number of tables required to cover potential discount rates that might be used by the analyst. Of course the private sector analyst may use the UPW factors provided in Part I provided (1) the analysis is performed in constant dollars and (2) the desired discount rate is either $4.7 \%$ or 10\%.)

For further explanation of the use of these indices, see NBS Special Publication 709, appendix B, Part I.

Note: The data in the tables which follow are reported by 4 Census regions. Figure B-1 presents a map showing the states corresponding to the 4 Census regions. The Census regions do not include American Samoa, Canal Zone, Guam, Puerto Rico, Trust Territory of the Pacific Islands, or the Virgin Islands. Analysts of Federal projects in these areas should use data which are "reasonable under the circumstances," and may refer to the tables with U.S. average data for guidance. 


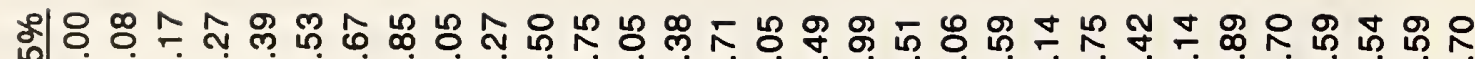

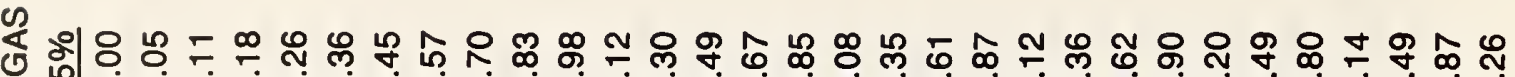

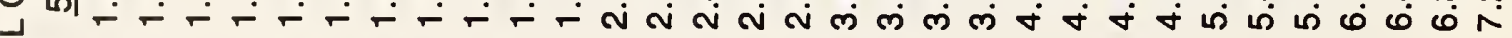
霖

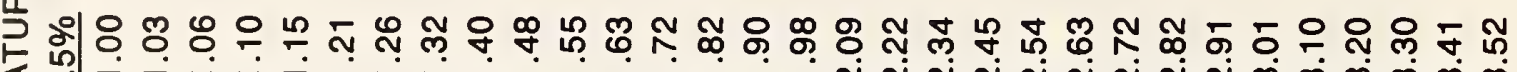
¿

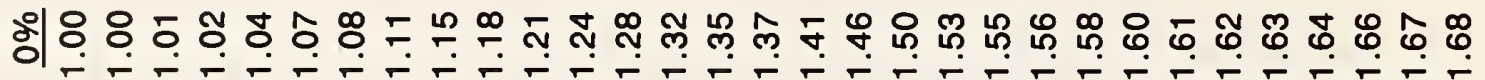
舫

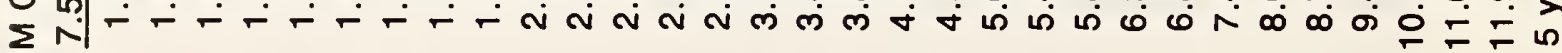

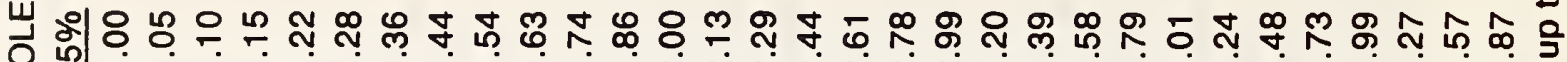
茫

原

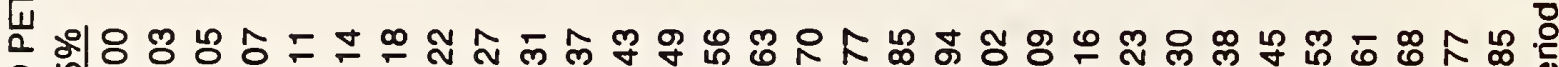
\lrcorner 互 $\ll \frac{1}{4}$

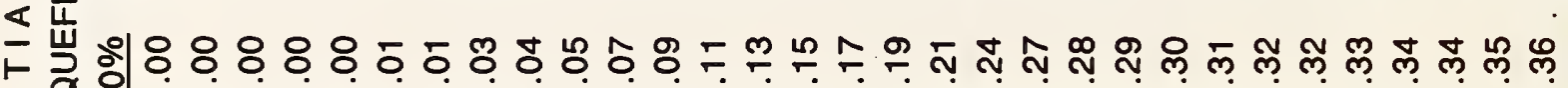
$z$ 음 山 $\frac{1}{\omega}$

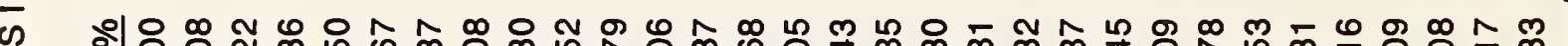
山 ద $\llbracket$ 崖 山N

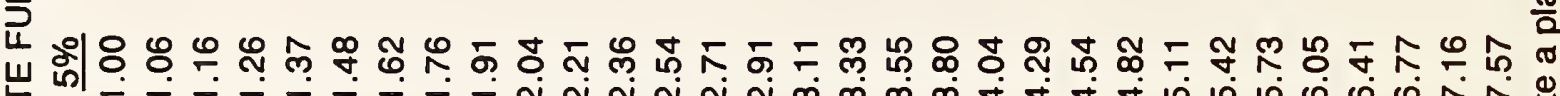

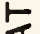

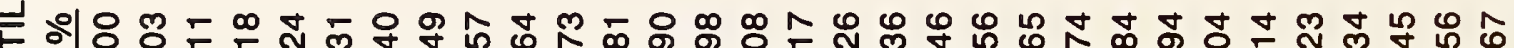

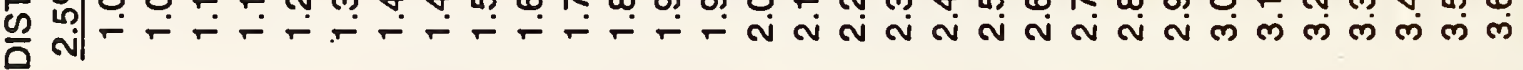

인

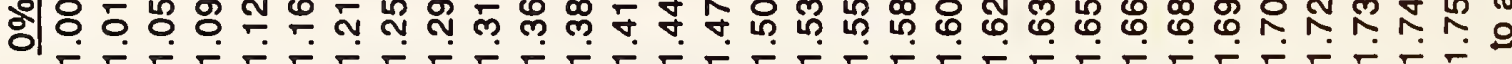
(1) 过 10.5 10 N ○

$\dot{9}$ .

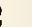




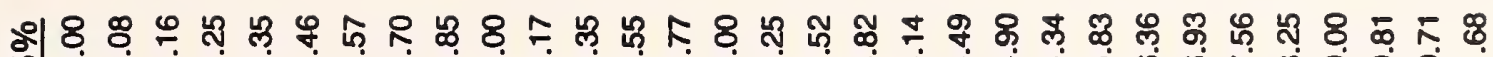
$\frac{1}{8}$

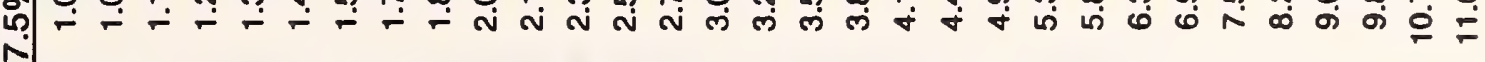

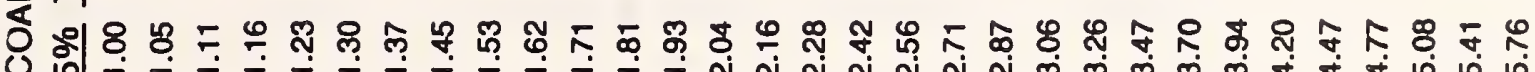
ט $\sum^{<}$

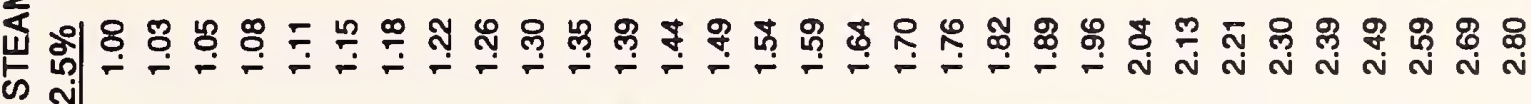

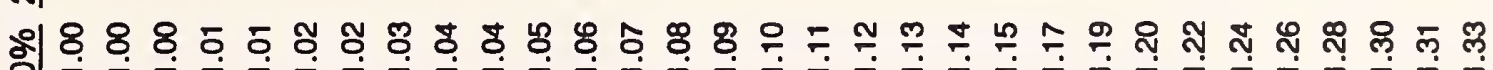

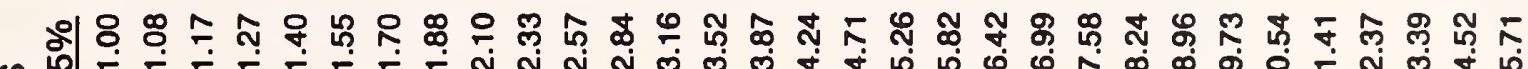

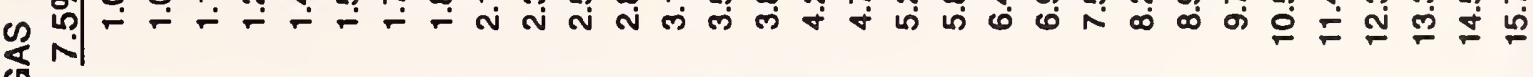

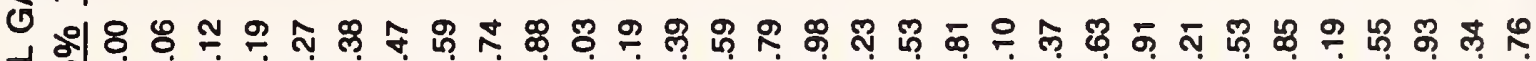
বर 왕 8 \% 8 누 ำ ลุ 乙 윙

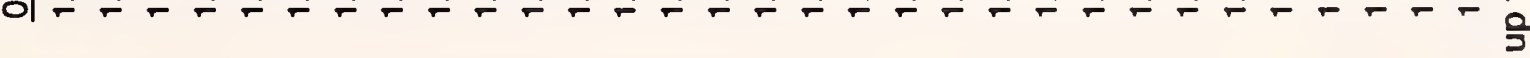

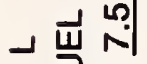

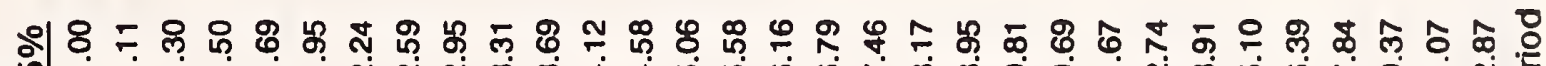

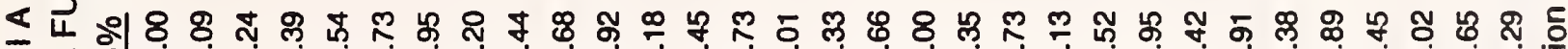
U 匹

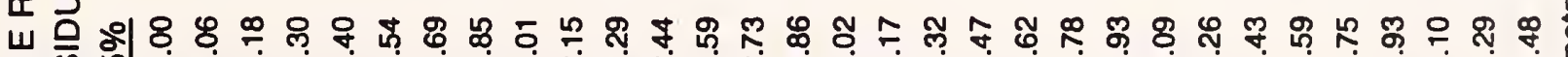
$\sum$ 湈 위 0 ठे|마-

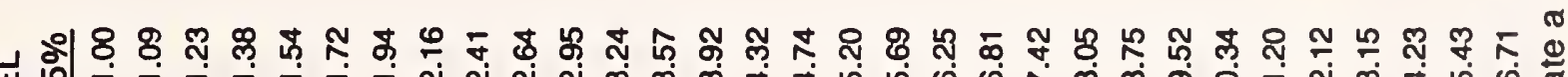
山ñ

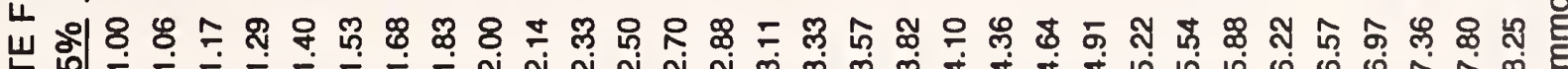
を

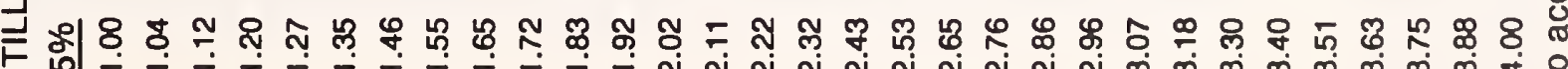
舟

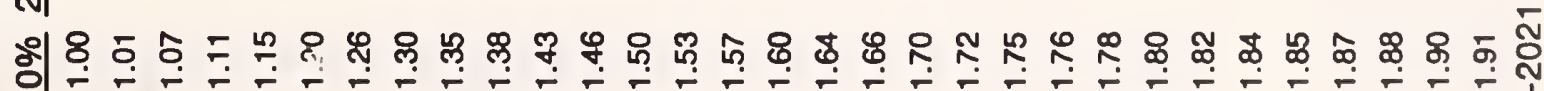

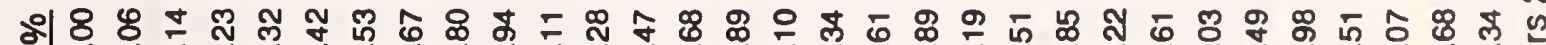
$>N$

|늠

化

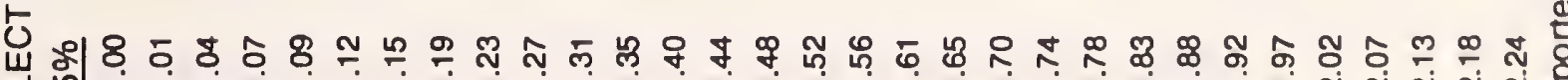
岃 왕 \% \% 利

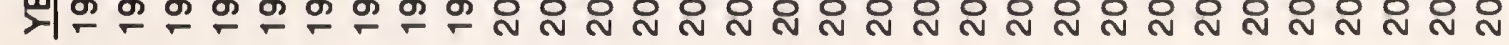




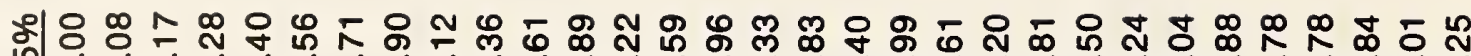

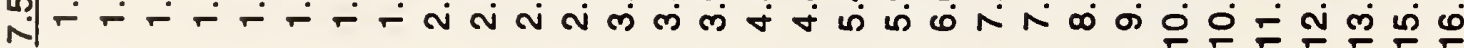

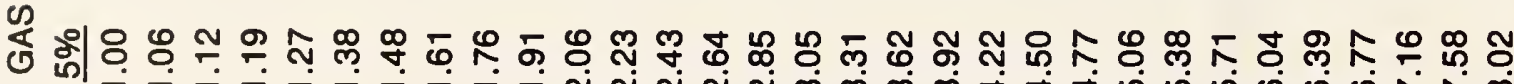

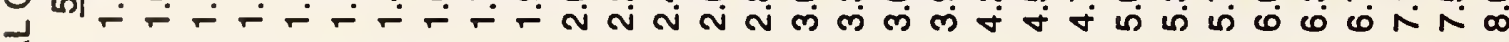
志

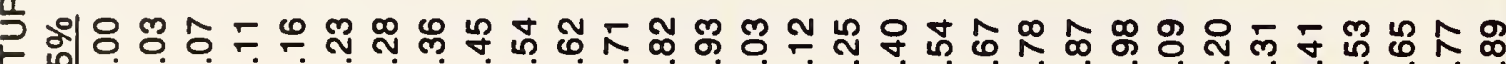

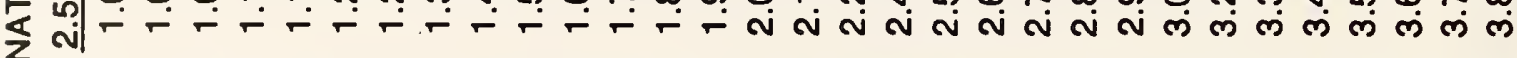

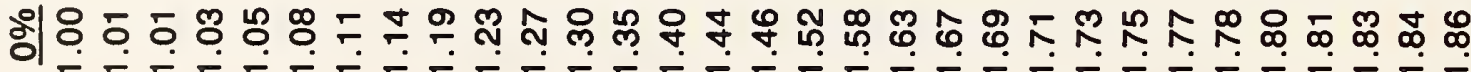

赵

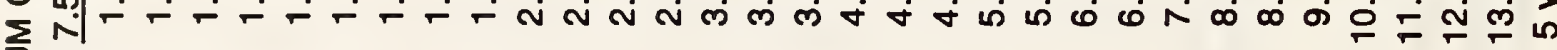

ய

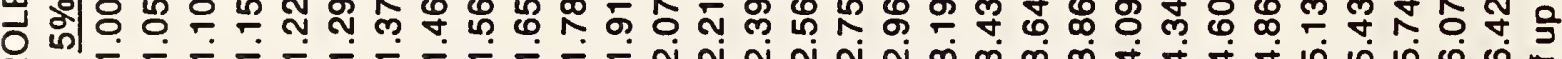
茫

$\overline{0}$

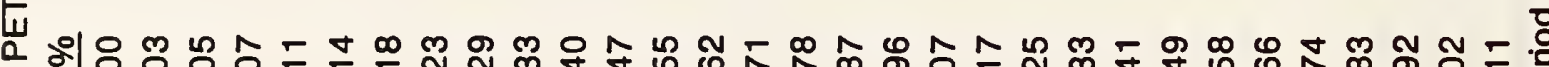
$\checkmark$ لـ 二 z $\underline{0}$ w

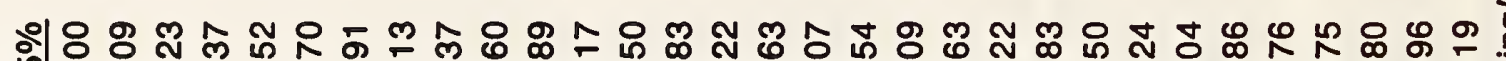
山 سلم 至

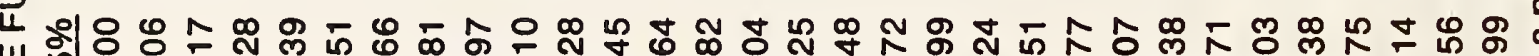

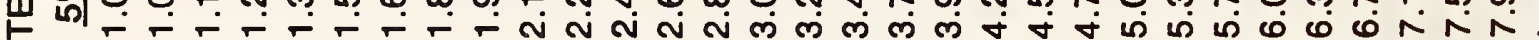

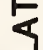

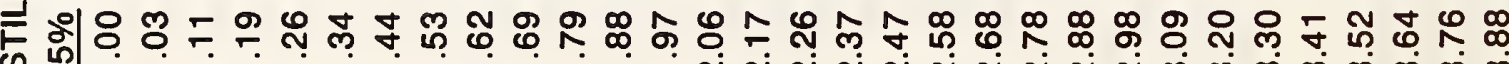
只

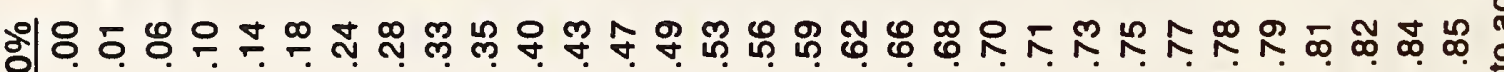

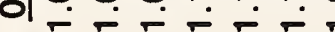

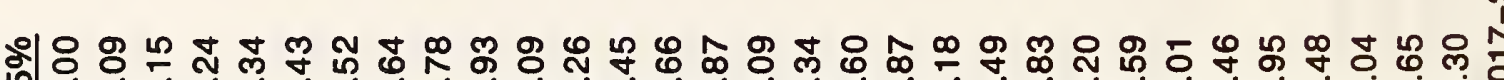

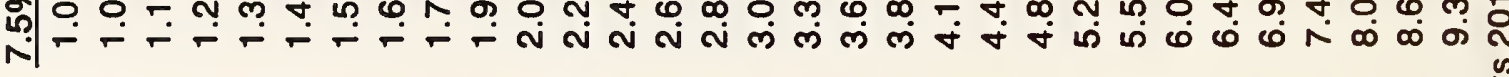

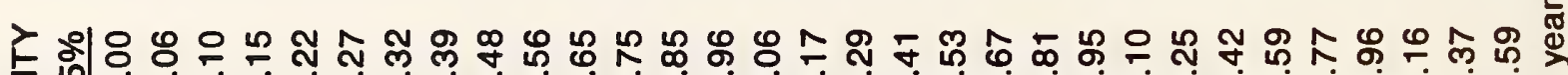
U 以l đ̃

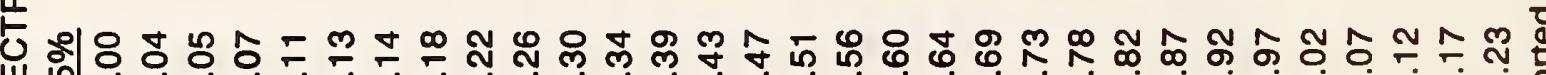
亗

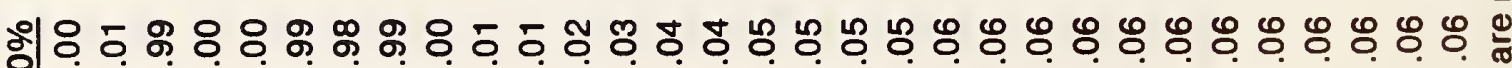

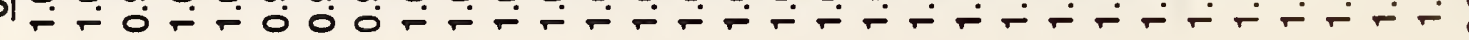

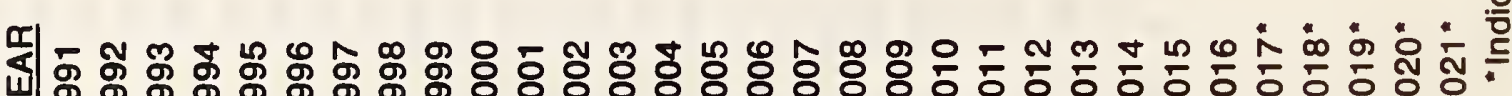

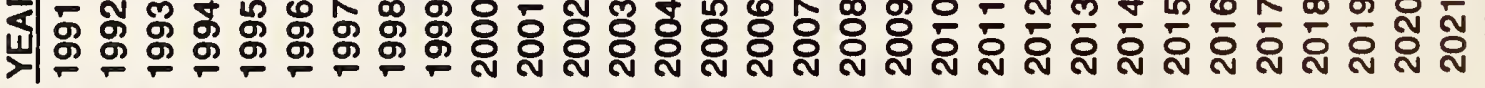


위 8 \% 운 ำ $\checkmark$ مी

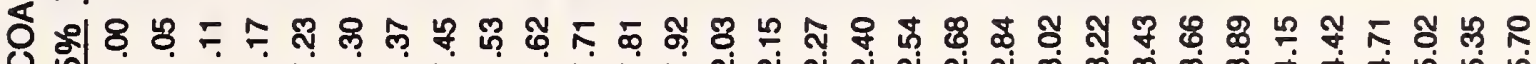
U $\sum$

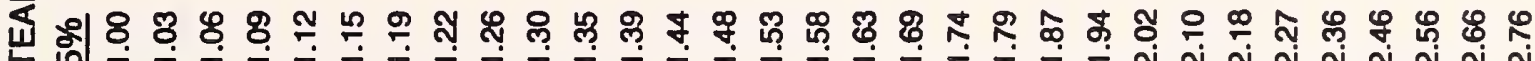
๘

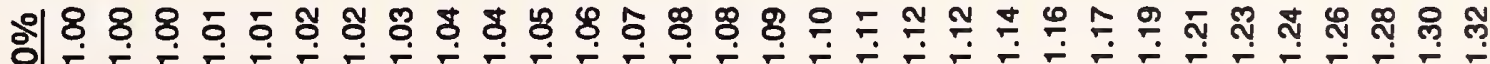

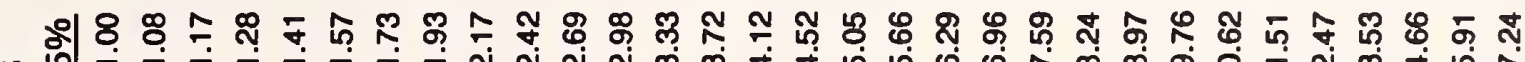
网

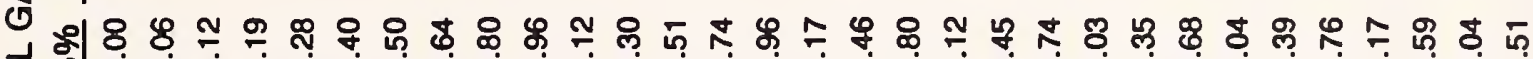

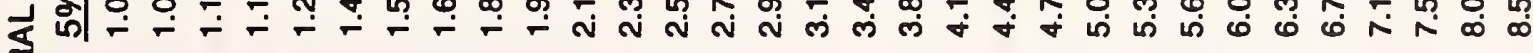
急

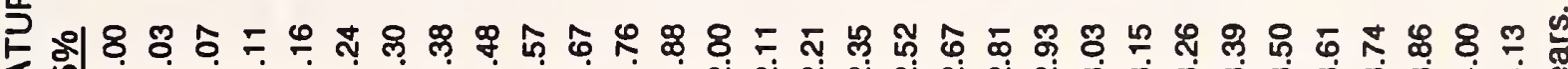
之

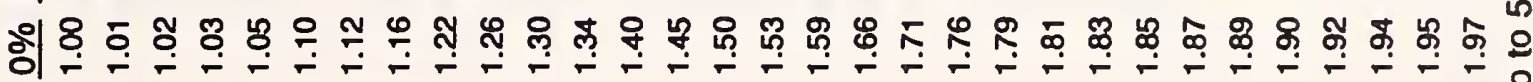

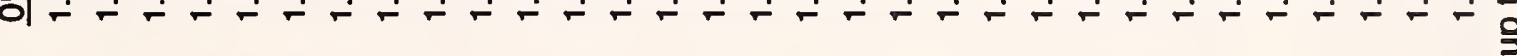

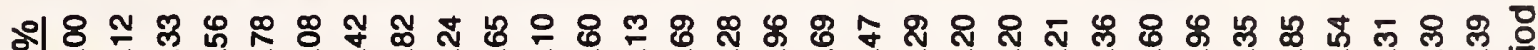
$\neg$ 岃

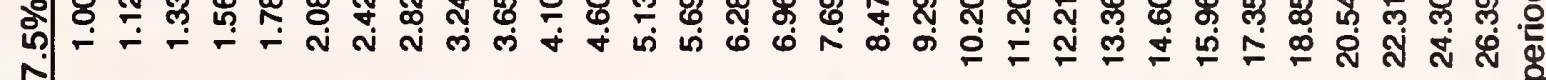

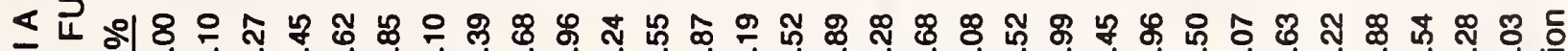

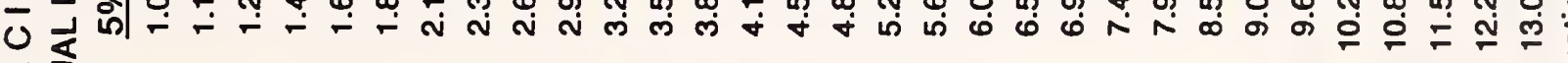

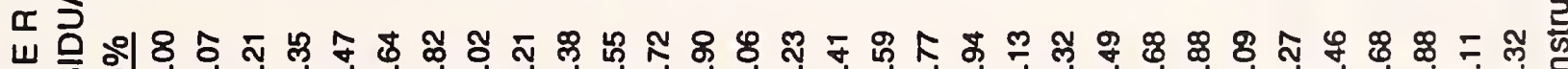
$\Sigma$ 岸 $\sum \pi$

0

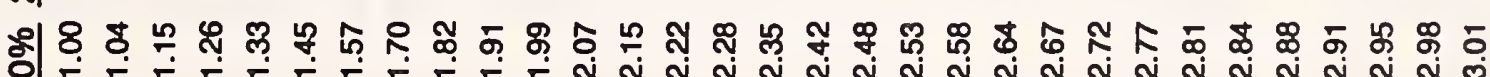
0

头 Ш

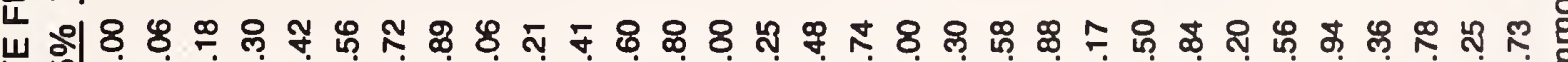
朋 $S$

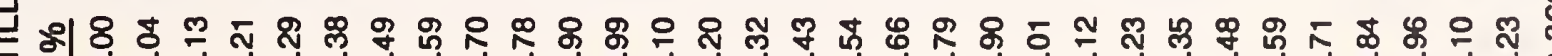

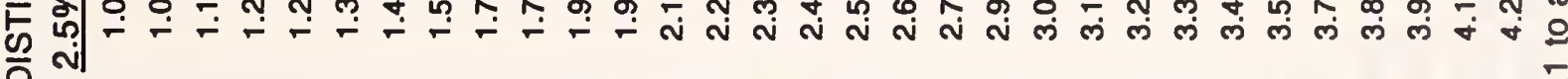

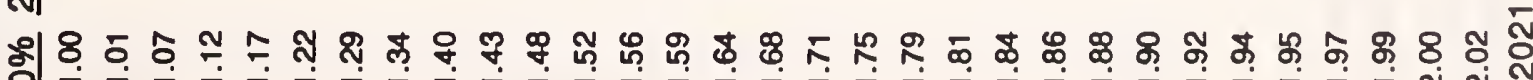
סำ

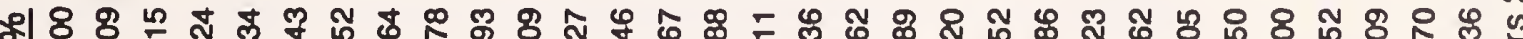
$>-1$

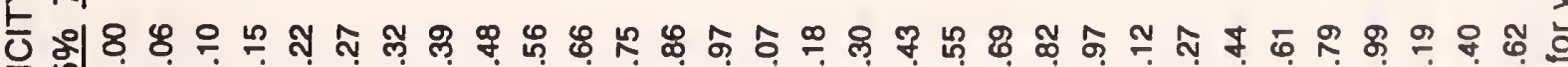
व

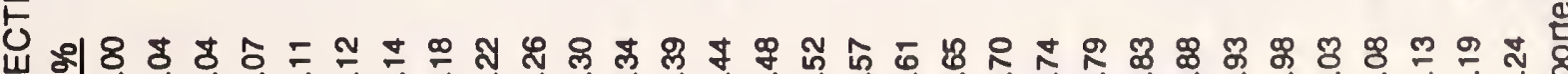
Ш 와웅

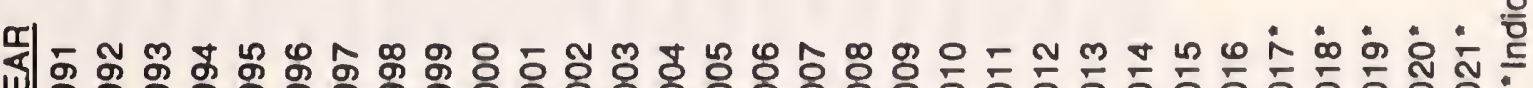

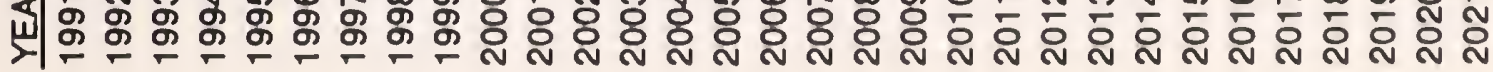




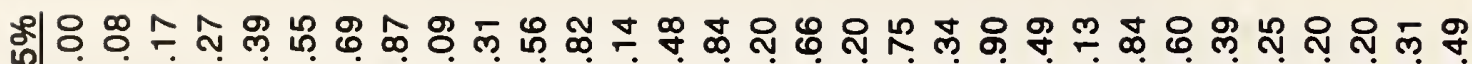
N-r- - (2)

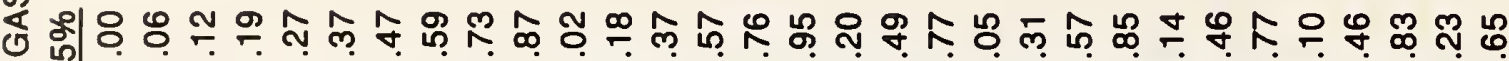

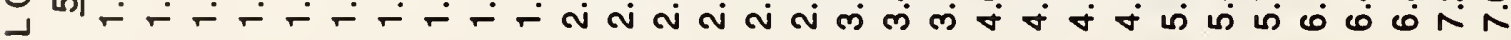

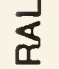

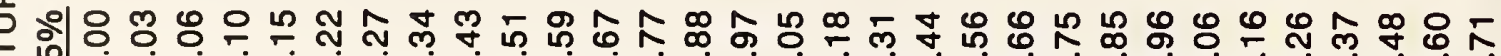

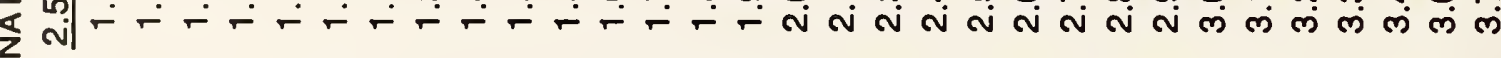

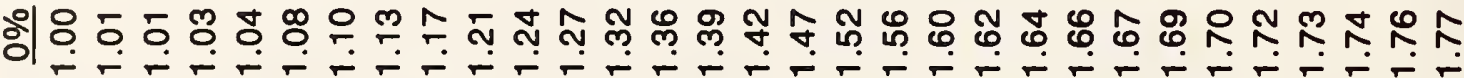

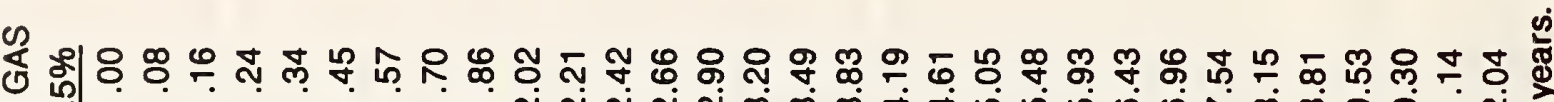

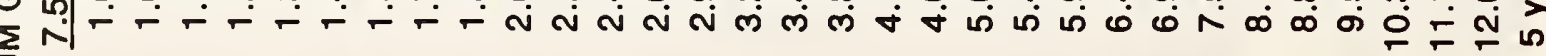

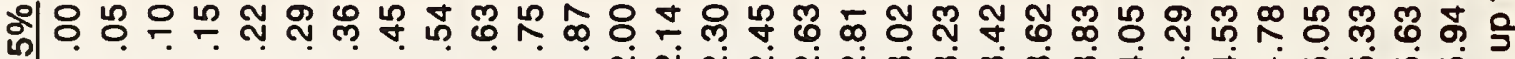
西

5

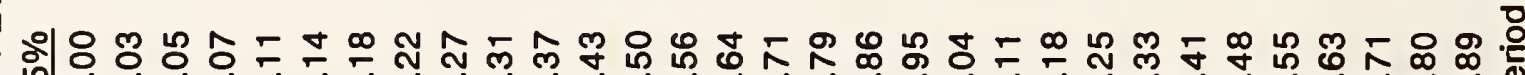
د 峛 $<\frac{11}{\text { II }}$

ㄴ $z$ O w

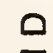

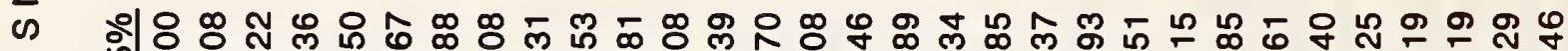
崩

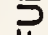

山ㅇํㅇㅇำ ลิ \&

윙 何

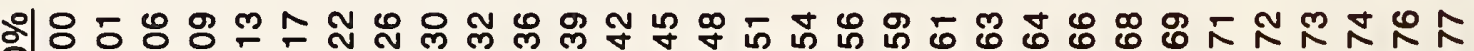
$10^{\circ}$

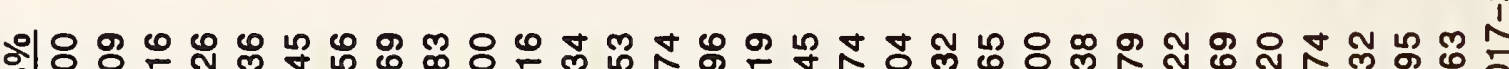
n़ी

乙 $\frac{0}{\bar{O}}$

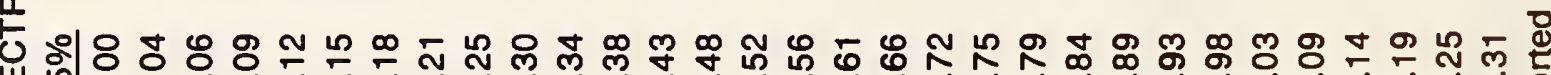
岂

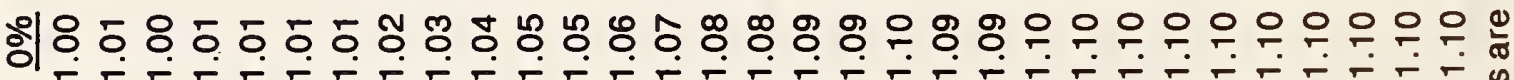

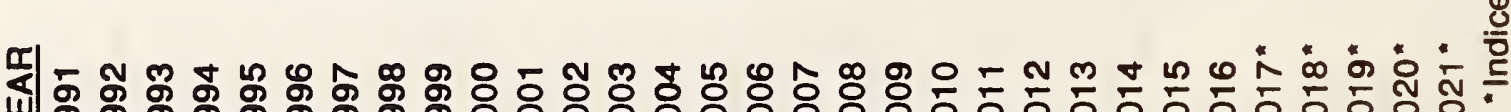

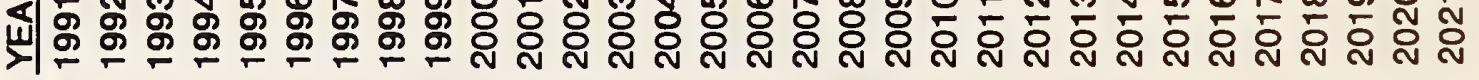




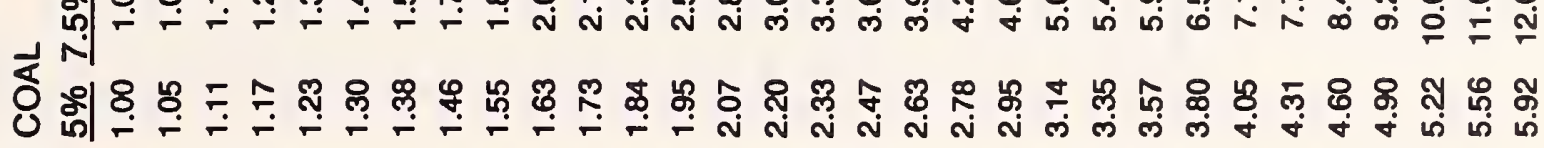

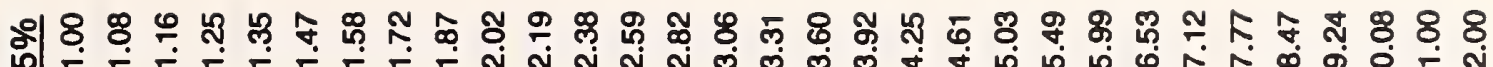
$\sum$

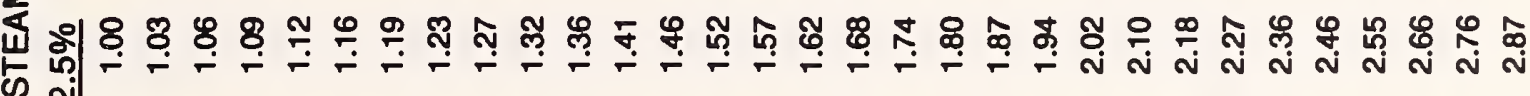

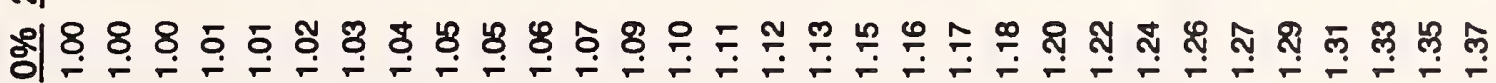

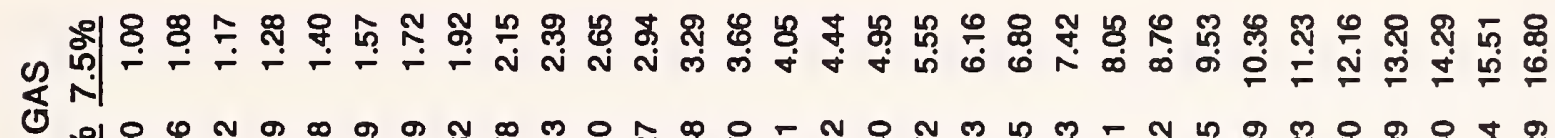

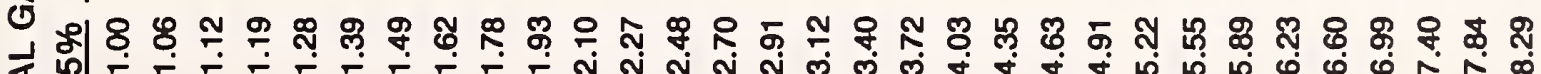
列

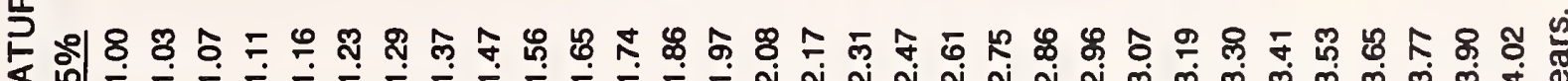

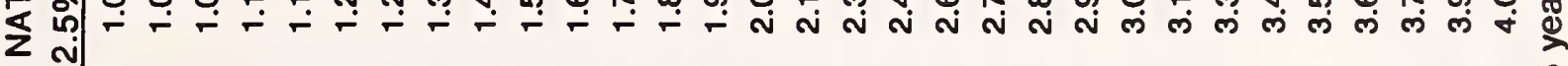

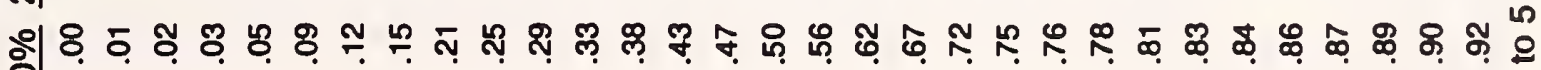
$\rightarrow$ 峲

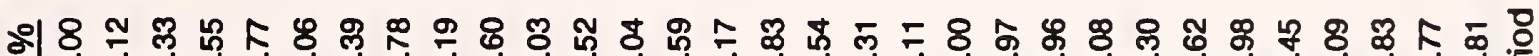

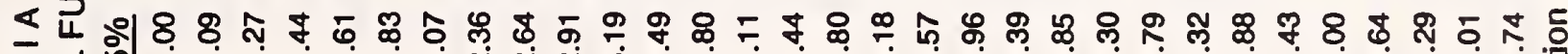

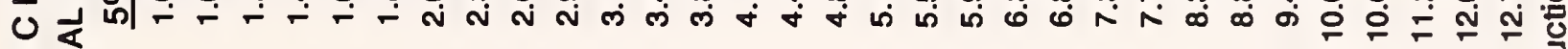

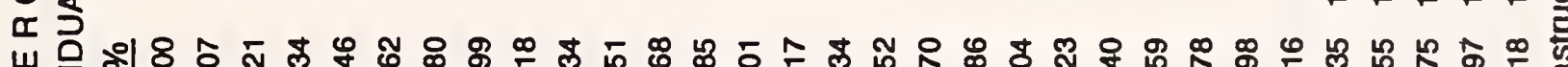

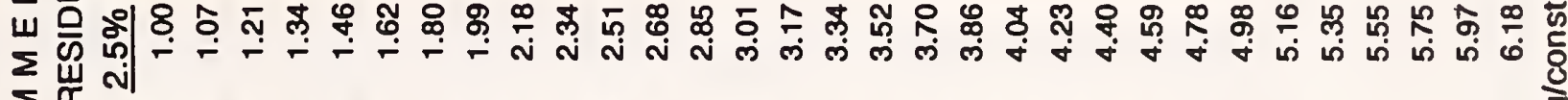

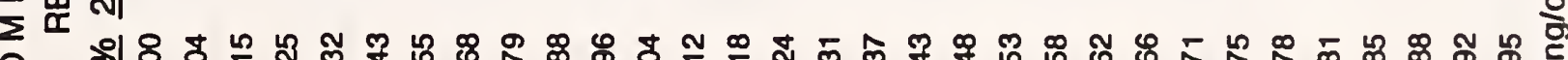

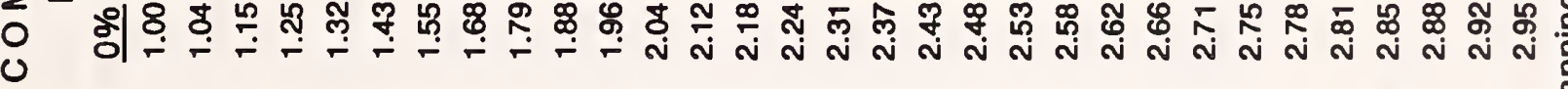

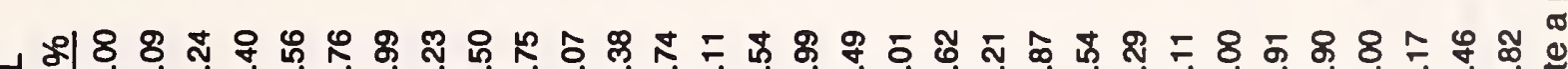

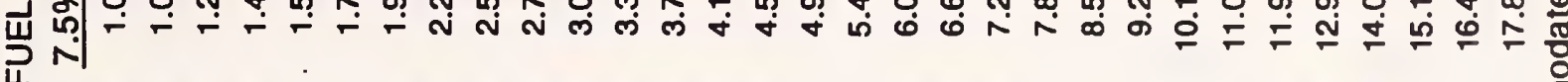
山4

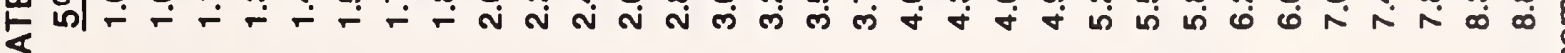

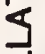

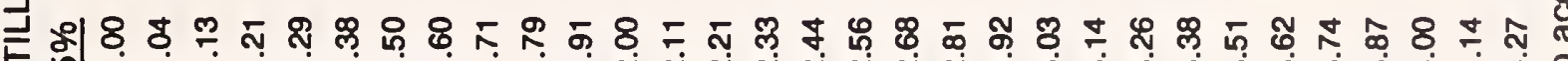

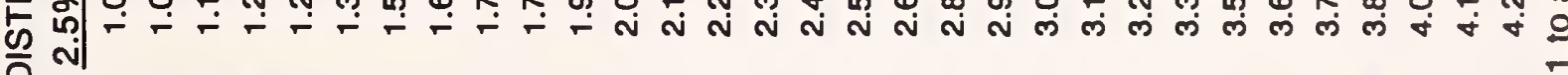

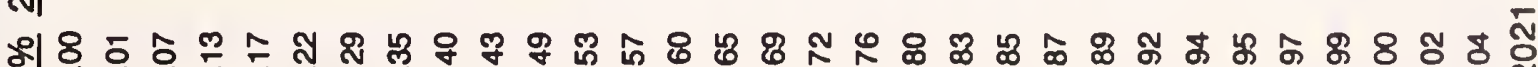
dนர்

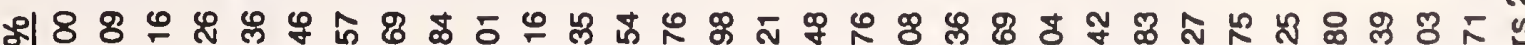

赑- - - - -

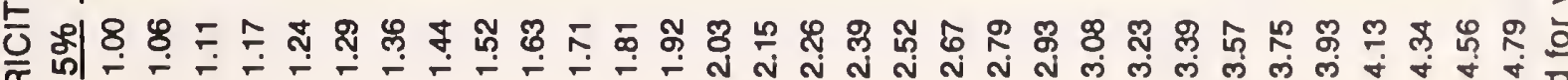

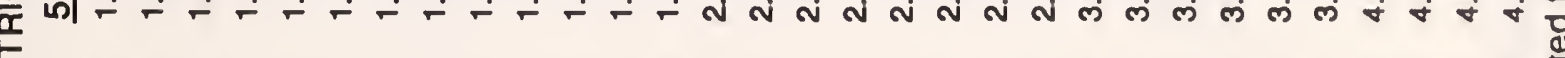
刿

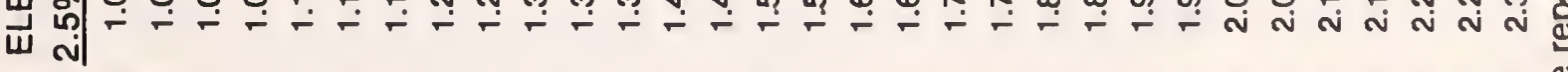

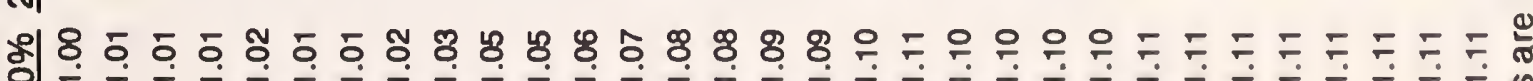

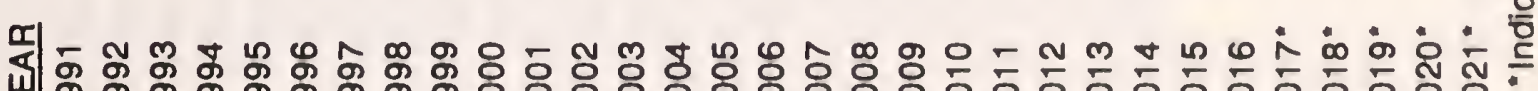

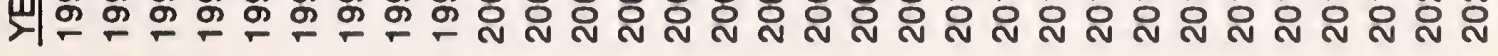




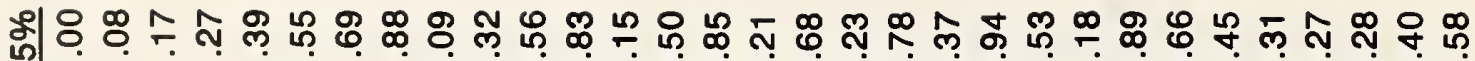
N|C)

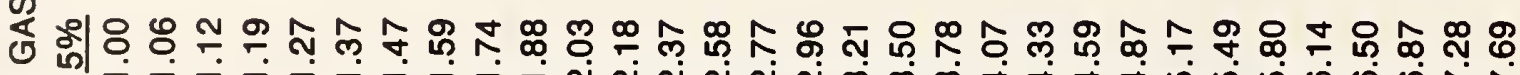
蛋

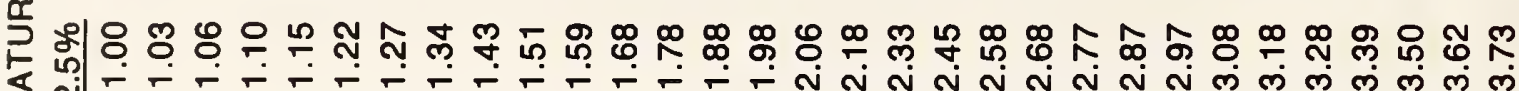
乏

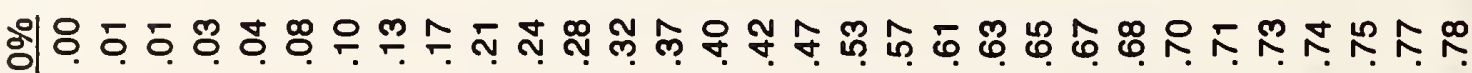

选

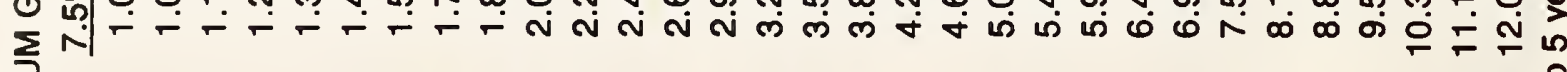

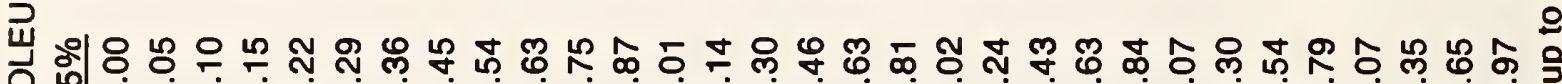
呬

tw

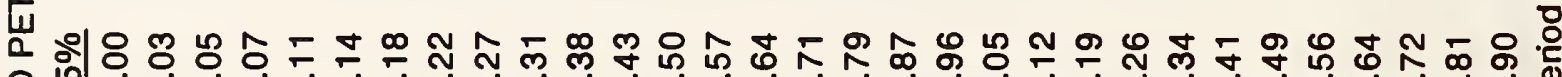
\lrcorner 岸

岂

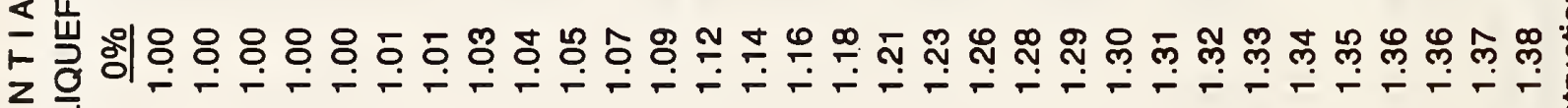
w

0

$\bar{c}$

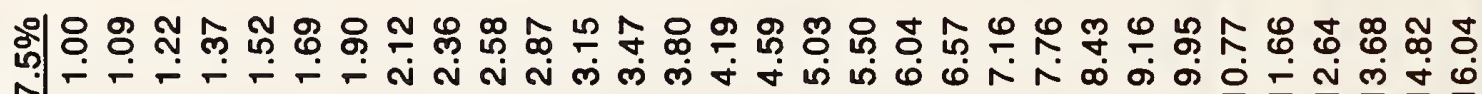

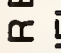

는의요

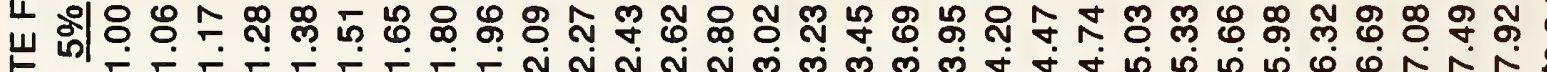
5

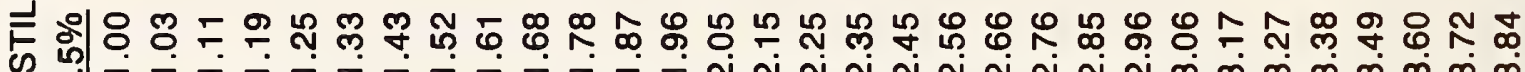

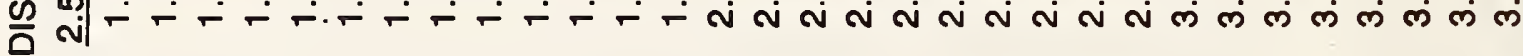

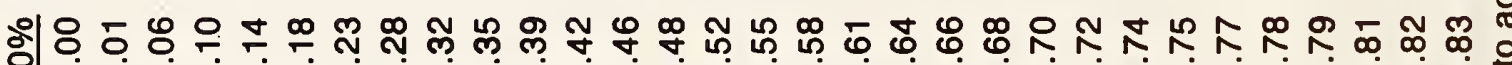

휭ㅇㅇㄴㄷำ

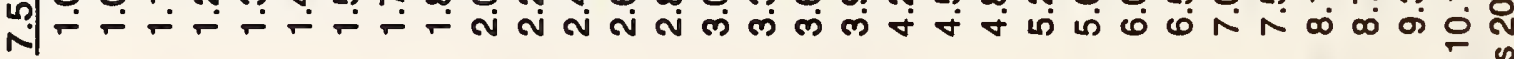

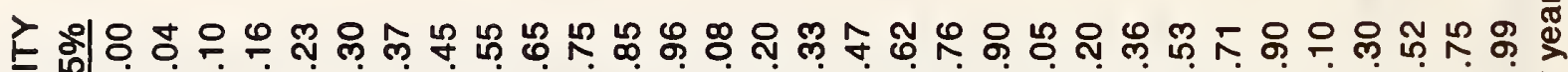

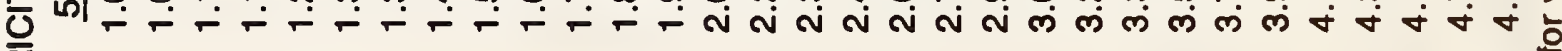
풍

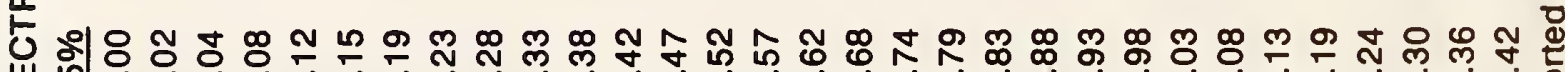
岃

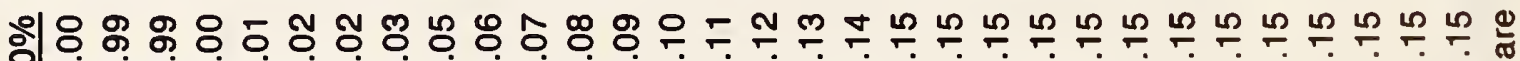

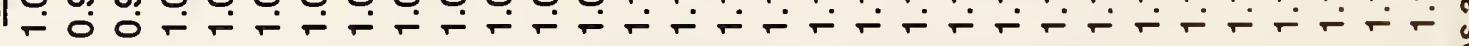

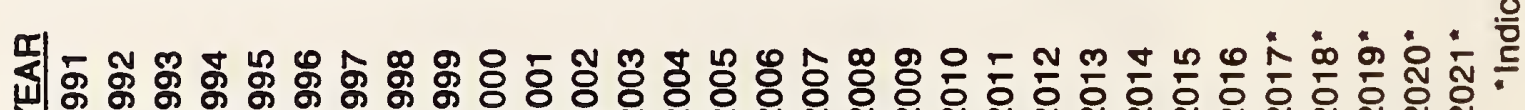

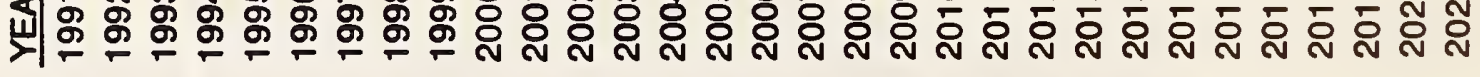




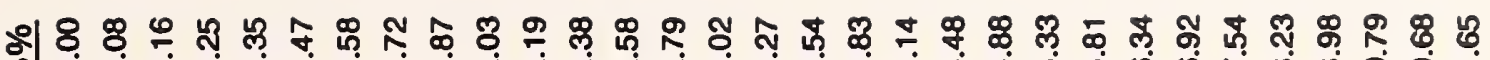
约舟

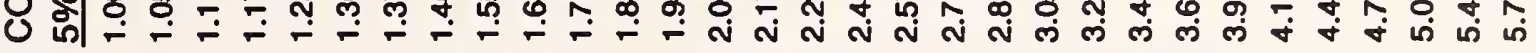
$\sum$ 志乩

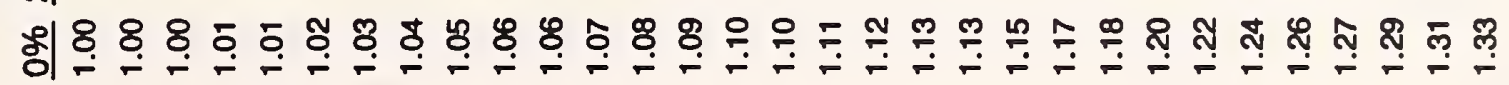

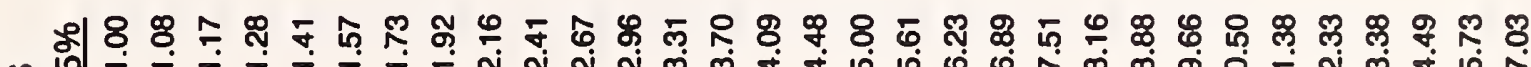
ठิ 卉部 $\frac{\alpha}{2}$

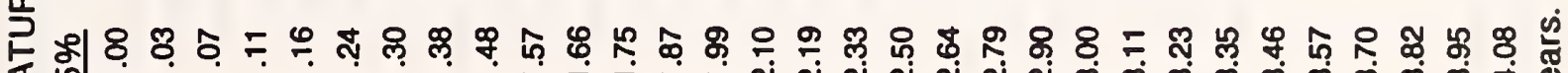

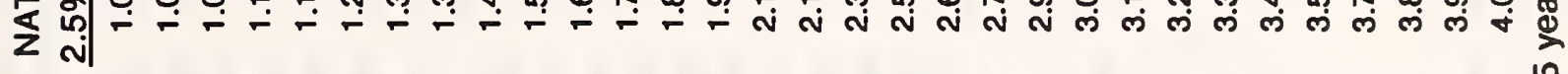

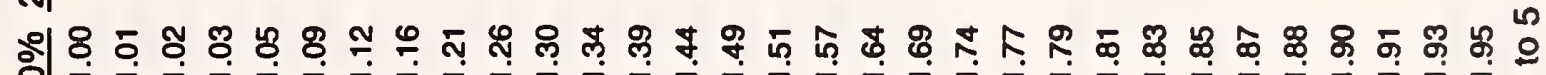
$\overrightarrow{\mathrm{U}}$

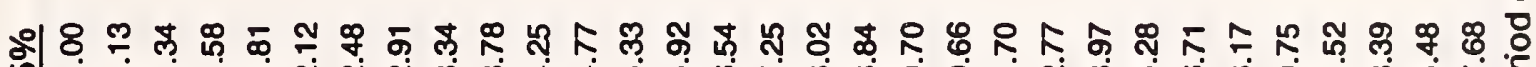

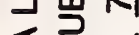

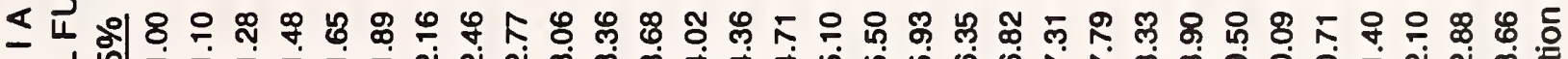
U

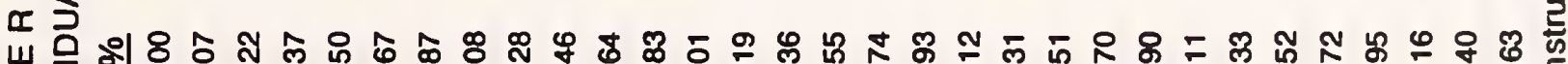
还岸

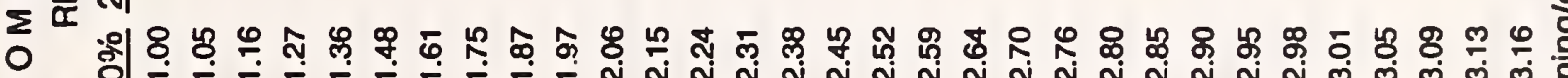
0 ol

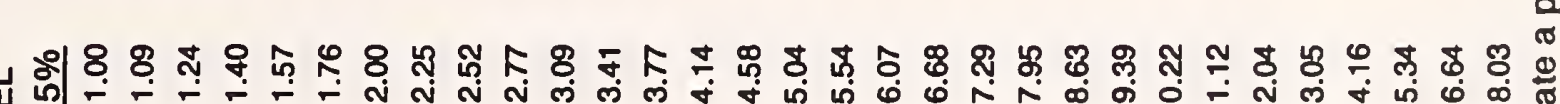
岃

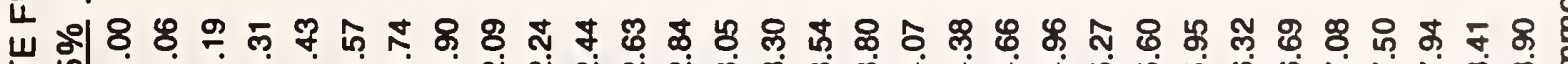

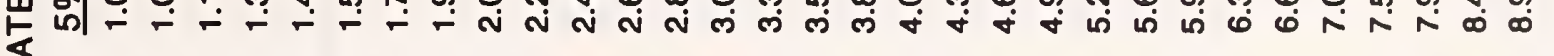

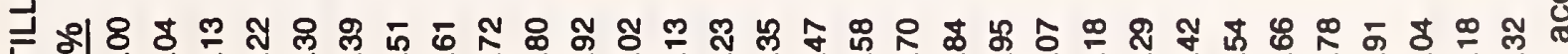
馬

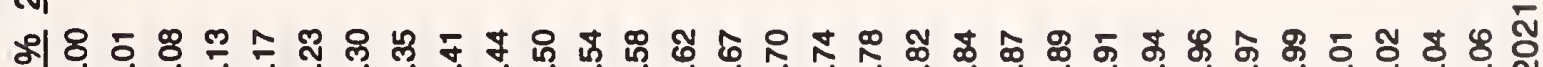

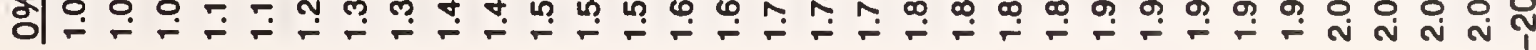

잉ำ $\tau \mathrm{N}$

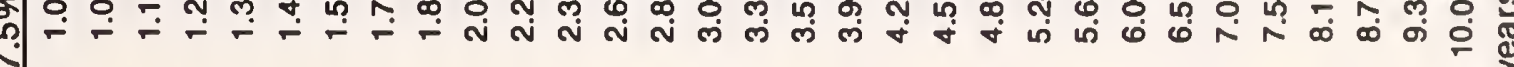

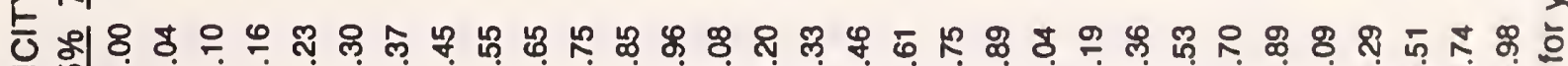
花

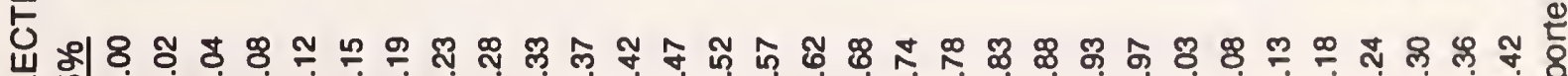
岃

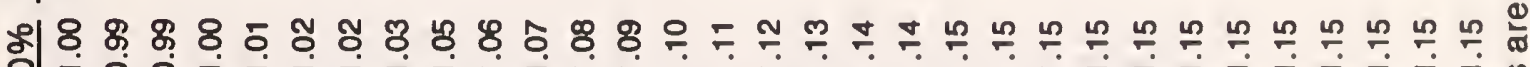
象

䄮

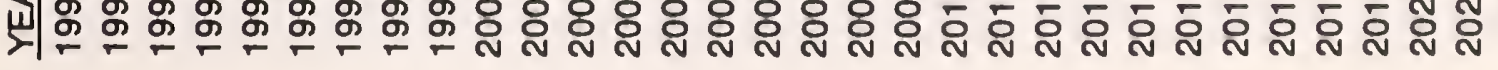


윙ㅇㅇㅇㄷㅅำ 负|( )

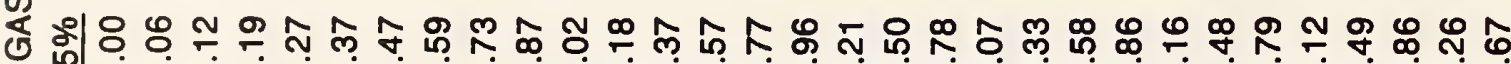
孚 윈 z

윙우웅 ช

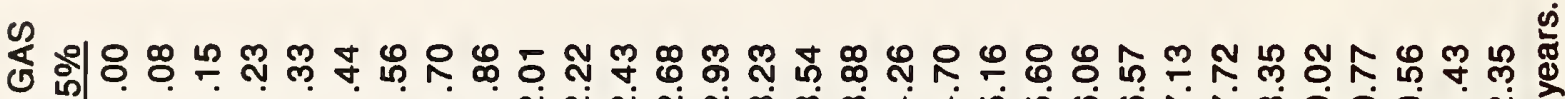

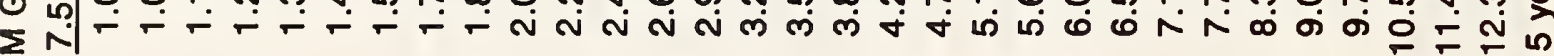

ய 이은

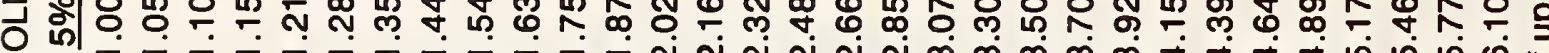
व

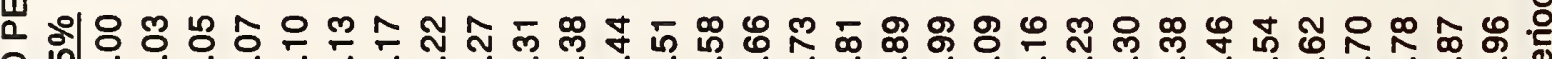
$\neg$ 山ै山l $<$

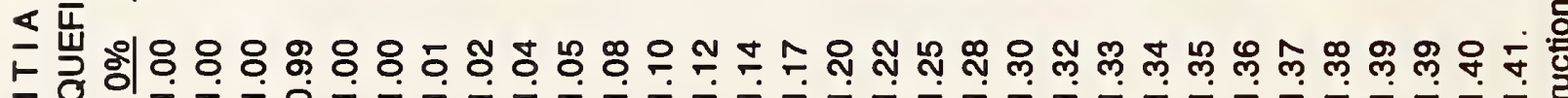

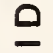

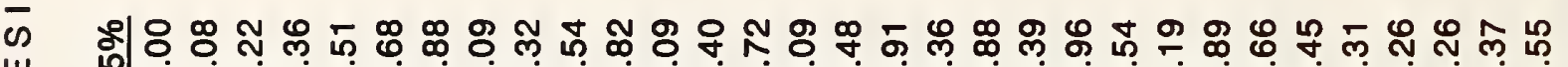

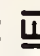

ய

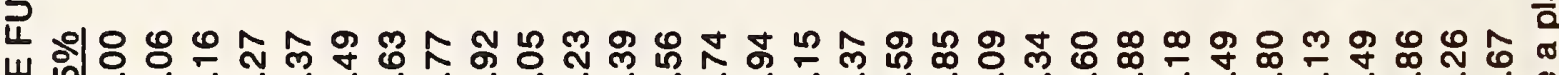
W 质

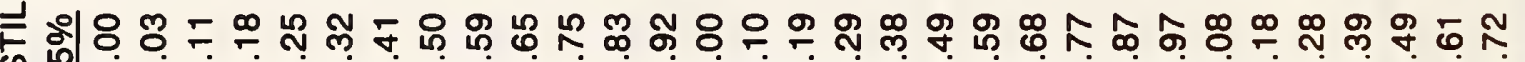
号

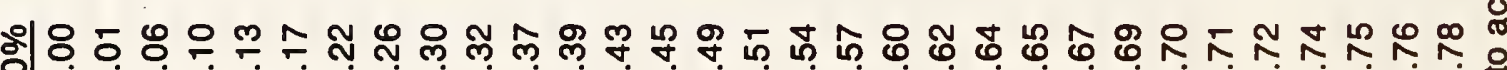

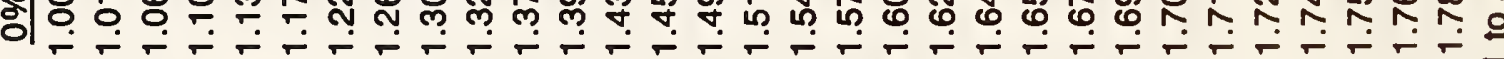

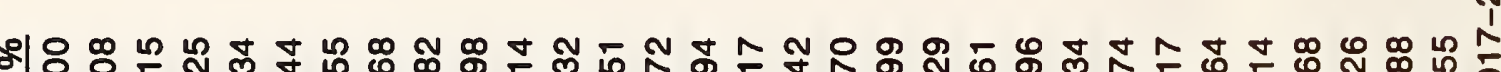

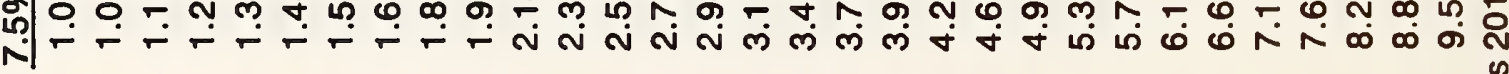

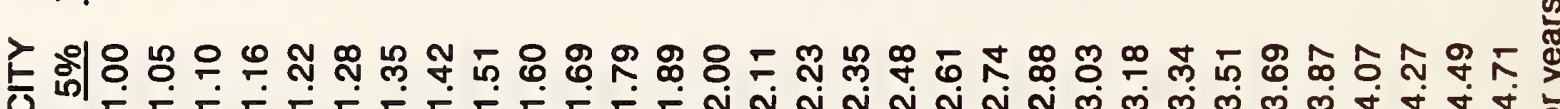

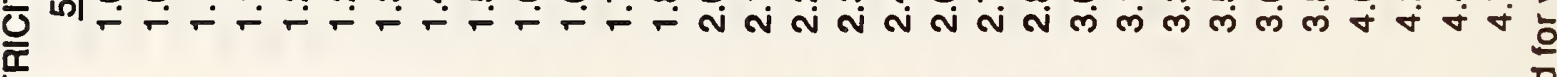

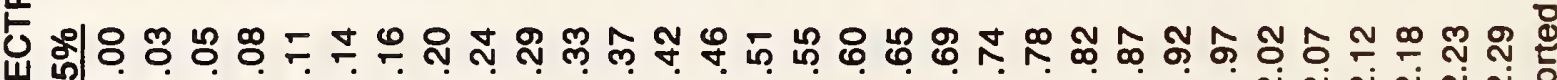
岃

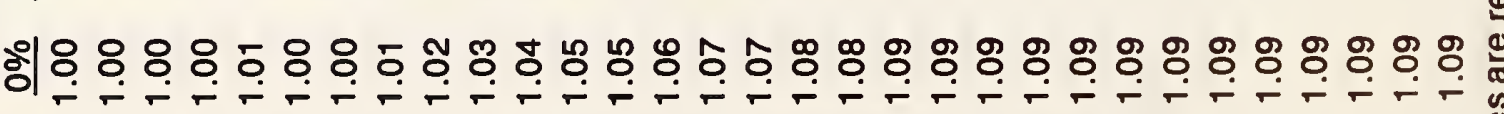

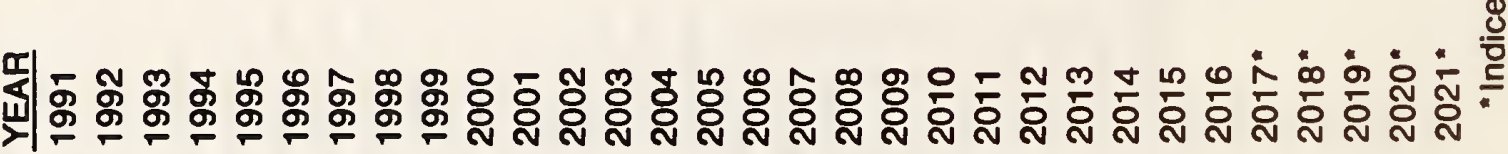




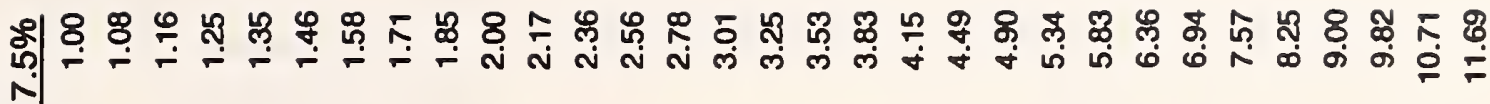
$\perp$ N

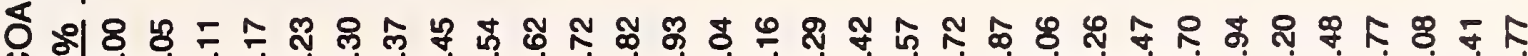

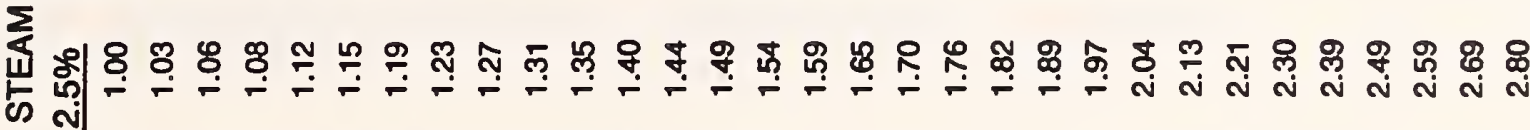

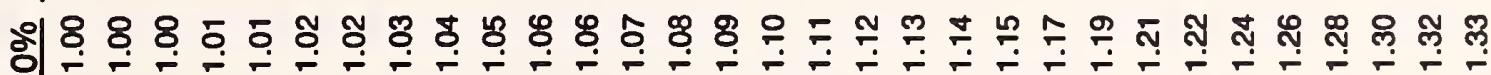

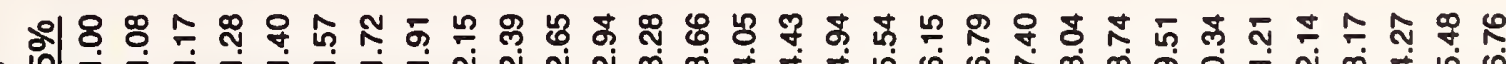
约 速 艺迕

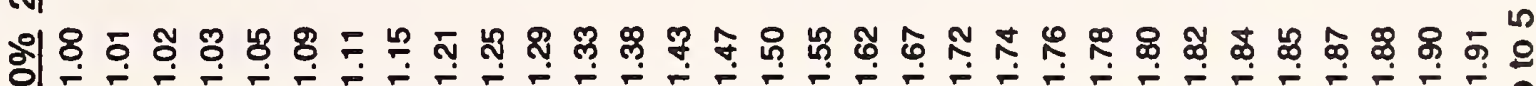

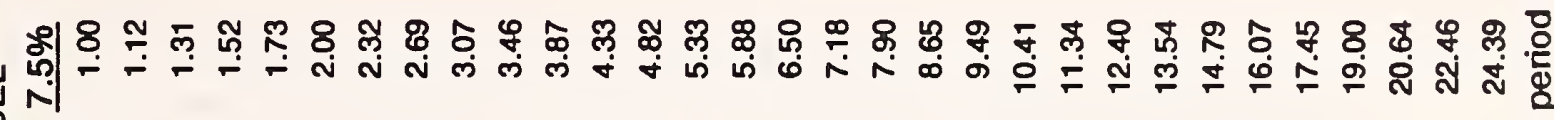
$\rightarrow$ Ш山े

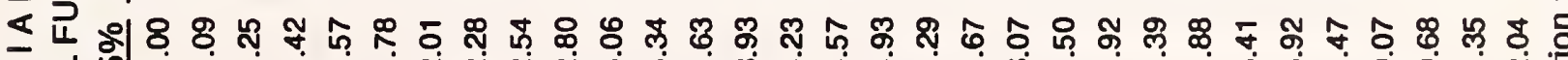
U

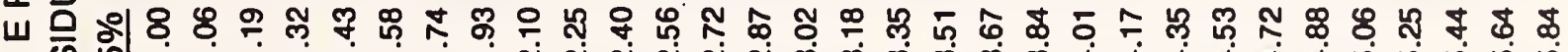
$\sum \bar{D}$ $\sum$ 压

0

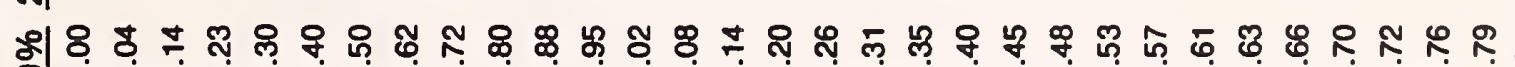

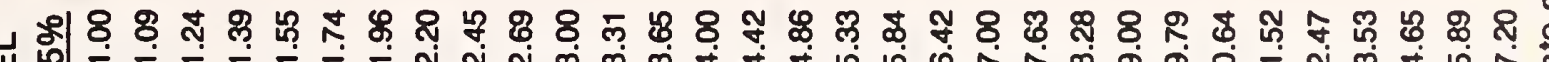
山 윙요 \& - - - - -

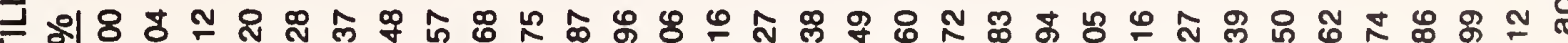
产 ஸู|

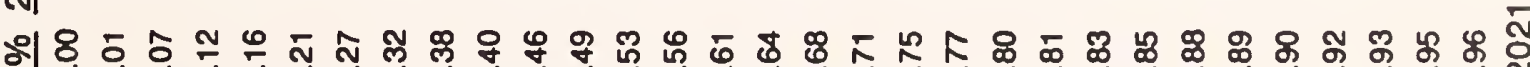

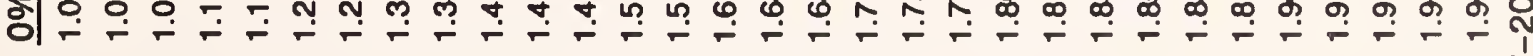

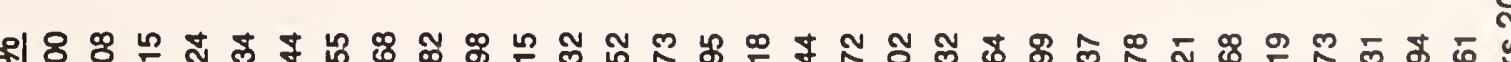
匇

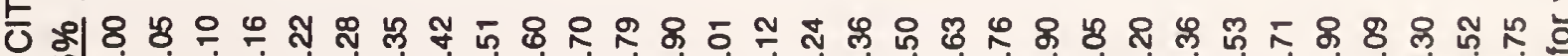
夰

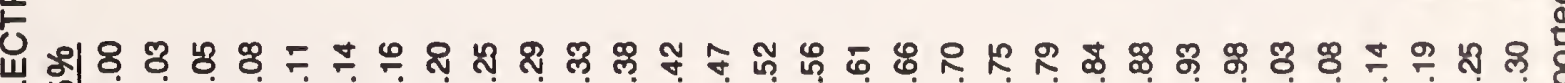
岃

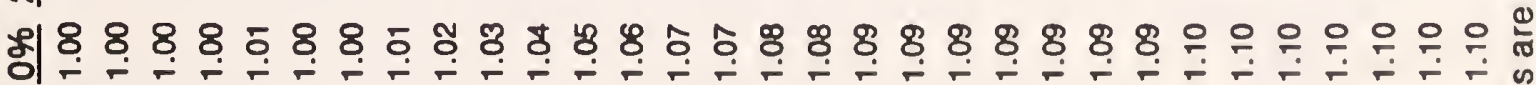

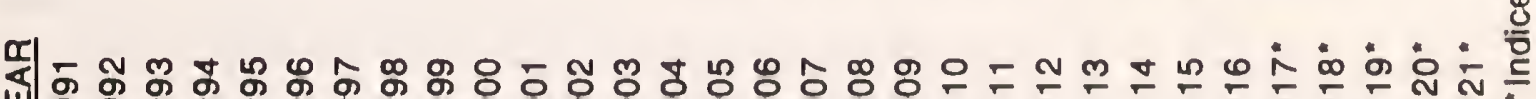

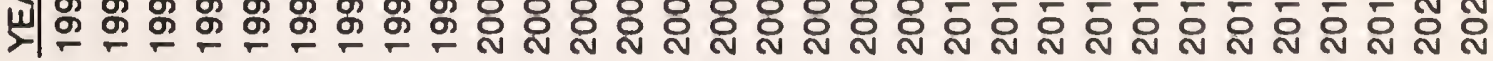


NBS.114A (REV. 2-8C)

U.S. OEPT. OF COMM.

BIBLIOGRAPHIC DATA

SHEET (See instructions)

1. PUBLICATION OR
REPORT NO.
NISTIR-85-3273-5

2. Performing Organ. Report No.

3. Pubilication Date

October 1990

4. TITLE AND SUBTITLE

Energy Prices and Discount Factors for Life-Cycle Cost Analysis 1991: Annual Supplement to NIST Handbook 135 and NBS Special Publication 709.

5. $A U T H O R(S)$

Barbara C. Lippiatt and Rosalie T. Ruegg

6. PERFORMING ORGANIZATION (If joint or other than NBS. see in structions)

MATIONAL INSTITUTE OF STANDARDS AND TECHNOLOGY

(formorly NATIONAL BUREAU OF STANDARDS)

U.S. DEPARTMENT OF COMMERCE

GATHERSBURG, MD 20890

7. Contract/Grant No.

9. SPONSORING ORGANIZATION NAIME ANO COMPLETE ADDRESS (Street, City, StOte, ZIF)

U.S. Department of Energy

office of the Assistant Secretary

Federal Energy Management Program

for Conservation and Renewable Energy

Washington, DC 20585

10. SUPPLEMENTARY NOTES

This report is comprised of energy price and discount factor tables which are annual updates of tables referenced in NIST Handbook 135 and NBS Special Publ1cation 709. Only minor changes have been made to this previously WERB-approved material.

$\square$ Document describes a computer program; SF-185, FIPS Software Summary, is attached.

11. ABSTRACT (A 200-word or less factual summary of most significant information. If document includes a significant bibliography or literature survey. mention it here)

This is the 1991 annual edition of energy prices and discount factors for performing life-cycle cost analyses of energy conservation and renewable energy projects. It supports the Federal life-cycle costing methodology by updating the energy price projections and discount factors that are described, explained, and illustrated in NIST Handbook 135 (HB 135). It supports private-sector life-cycle cost analysis by updating the energy price indices that are described, explained, and illustrated in NBS Special Publication 709 (SP 709). It also supports the Energy Conservation Mandatory Performance Standards for New Federal Residential Buildings (10 CFR 435) by providing a table of factors for updating appliance label values.

12. KEY WORDS (Six to twelve entries; alphabet/cal order; capitallze only proper names; and separate key words by semicolons) average fuel prices; energy conservation; energy price forecasts; Federal Energy Management Program; life-cycle cost analysis; single present worth discount factors; uniform present worth discount factors

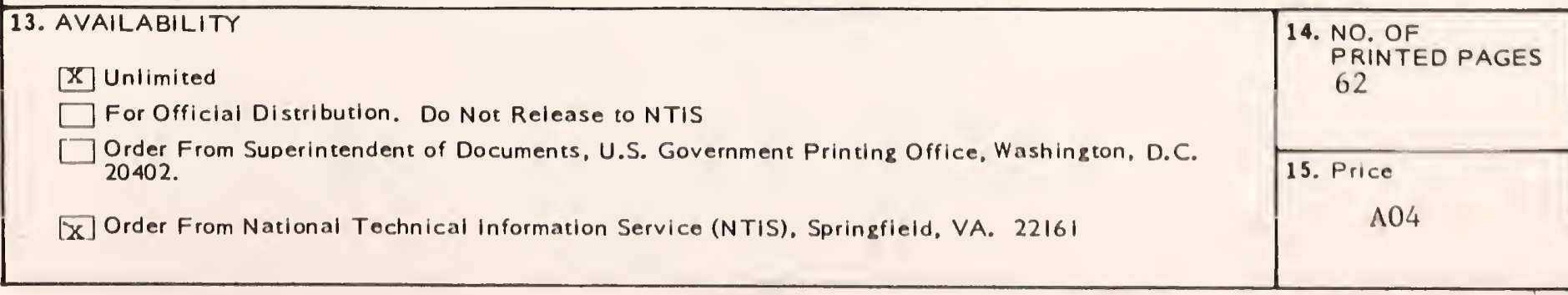




